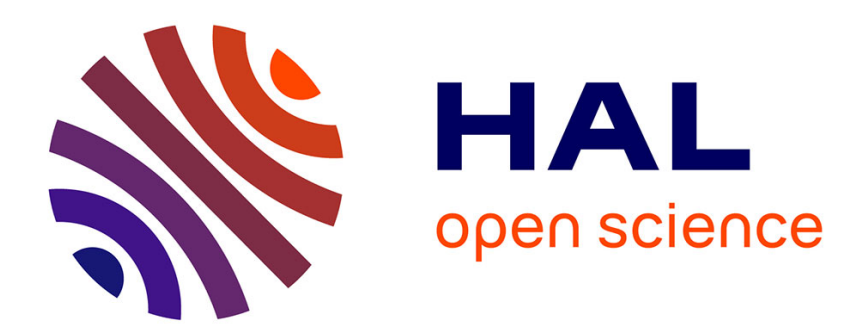

\title{
Prescribing the Jacobian in critical spaces
}

Pierre Bousquet, Petru Mironescu

\section{To cite this version:}

Pierre Bousquet, Petru Mironescu. Prescribing the Jacobian in critical spaces. Journal d'analyse mathématique, 2014, 122, pp.317-373. hal-00747444

\section{HAL Id: hal-00747444 \\ https://hal.science/hal-00747444}

Submitted on 31 Oct 2012

HAL is a multi-disciplinary open access archive for the deposit and dissemination of scientific research documents, whether they are published or not. The documents may come from teaching and research institutions in France or abroad, or from public or private research centers.
L'archive ouverte pluridisciplinaire $\mathbf{H A L}$, est destinée au dépôt et à la diffusion de documents scientifiques de niveau recherche, publiés ou non, émanant des établissements d'enseignement et de recherche français ou étrangers, des laboratoires publics ou privés. 


\title{
Prescribing the Jacobian in critical spaces
}

\author{
Pierre Bousquet * Petru Mironescu ${ }^{\dagger}$
}

July 12,2012

\begin{abstract}
We consider the Sobolev space $X=W^{s, p}\left(\mathbb{S}^{m} ; \mathbb{S}^{k-1}\right)$. We prove the existence of a robust distributional Jacobian $J u$ for $u \in X$ provided $s p \geq k-1$; this generalizes a result of Bourgain, Brezis and the second author [10], where the case $m=k$ is considered. In the critical case where $s p=k-1$, we identify the image of the map $X \ni u \mapsto J u$. This extends a result of Alberti, Baldo and Orlandi [2] for $s=1$ and $p=k-1$. We also present a new, analytical, dipole construction method.
\end{abstract}

\section{Contents}

1 Introduction 1

2 Sobolev spaces of currents and forms 6

3 Rectifiability, area and coarea $\quad 8$

4 Interpolation inequalities $\quad 10$

5 Proof of Theorems 1.1 and $1.2 \quad 12$

6 Proof of Theorem 1.3 21

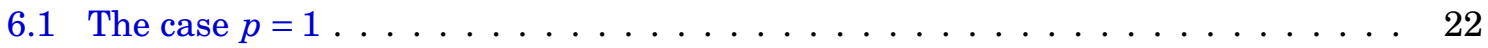

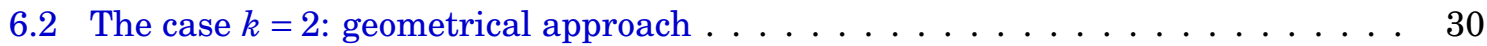

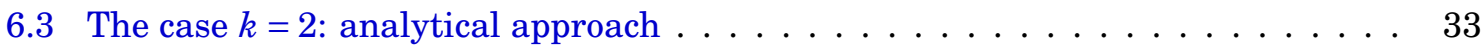

7 Proof of Lemma 5.1 $\quad 37$

\section{Introduction}

In general, one cannot approximate manifold-valued Sobolev maps with manifold-valued smooth maps. This contrasts with the case of scalar maps. Here is a simple example, with $\mathbb{D}$ the unit disc in $\mathbb{R}^{2}$ : the map $u_{0}: \mathbb{D} \rightarrow \mathbb{S}^{1}, u_{0}(z)=\frac{z}{|z|}$, belongs to $W^{1,1}$, but cannot be approximated in $W^{1,1}$ with smooth $\mathbb{S}^{1}$-valued maps [37]. Beyond the approximation problem, singularities occur in the regularity theory of manifold-valued maps (developed by Schoen and Uhlenbeck [37, 38] and extended by Hardt and Lin [24]): unlike the scalar case, manifold-valued minimizers of the Dirichlet integral $\int|\nabla u|^{2}$ or more generally of $\int|\nabla u|^{p}$ are not smooth, but have a (small) singular set. In the case of sphere-valued maps, Brezis, Coron and Lieb [13] generalized a

*Aix-Marseille Université; CNRS, UMR 7353, LATP, Centre de Mathématiques et Informatique, 39 rue Frédéric Joliot-Curie, F-13453 Marseille Cedex 13, France. Email address: bousquet@cmi.univ-mrs.fr

†Université de Lyon; Université Lyon 1; CNRS, UMR5208, Institut Camille Jordan, 43 blvd du 11 novembre 1918, F-69622 Villeurbanne-Cedex, France. Email address: mironescu@math.univ-lyon1.fr $\bowtie$ 
calculation of Ball [4] and discovered the possibility of detecting the singularities of maps via the distributional Jacobian. We will recall below the definition of the distributional Jacobian in the general case, but for the time being let us consider the case of maps $u: \mathbb{D} \rightarrow \mathbb{S}^{1}$. If $u \in C^{1}$, then the Jacobian determinant Jac $u$ satisfies

$$
\operatorname{Jac} u=0 .
$$

Indeed, by differentiating the identity $|u|^{2} \equiv 1$ we find that $u \cdot \partial_{j} u \equiv 0, j=1,2$, and thus $\partial_{1} u \| \partial_{2} u$. Therefore Jac $u=0$. Ball [4] discovered that for less smooth maps the Jacobian, when defined properly, need not vanish. Indeed, consider the distribution $J u=\frac{1}{2 \pi} \partial_{1}\left(u \wedge \partial_{2} u\right)+$ $\frac{1}{2 \pi} \partial_{2}\left(\partial_{1} u \wedge u\right),{ }^{1}$ which makes sense if $u$ is merely $W^{1,1} \cap L^{\infty}$. If $u \in C^{2}$, then $J u=\frac{1}{\pi} \mathrm{Jac} u$. Therefore, if $u$ is in addition circle-valued, then $J u$ vanishes as above. With more work, one may prove that the same holds if $u$ is less smooth, for example if $u \in W^{1,2}$. However, the above $u_{0}$ belongs to $W^{1, p}, p<2$, and it is easy to see that $J u=\delta_{0}$ [4]. ${ }^{2}$ More generally, if $u \in W^{1,1}\left(\mathbb{D} ; \mathbb{S}^{1}\right)$ is smooth outside a finite set $A(u)$, then [13]

$$
J u=\sum_{a \in A(u)} \operatorname{deg}(u, a) \delta_{a} .
$$

Here, $\operatorname{deg}(u, a)$ is the winding number (degree) of $u$ computed on a small circle around $a .^{3}$ It is still possible to compute the distribution $J u$ for an arbitrary $u \in W^{1,1}\left(\mathbb{D} ; \mathbb{S}^{1}\right)$, but this requires additional ingredients.

1. First ingredient: the class $\mathscr{R}$ of circle-valued maps $u$ smooth outside some finite set $A(u)$ is dense in $W^{1,1}\left(\mathbb{D} ; \mathbb{S}^{1}\right)$. This is a result of Bethuel and Zheng [8].

2. Second ingredient: for $u \in \mathscr{R}$, it is possible to rearrange the singularities of $u$ and write

$$
J u=\sum\left(\delta_{P_{j}}-\delta_{N_{j}}\right), \quad \text { with } P_{j}, N_{j} \in \overline{\mathbb{D}} \text { and } \sum\left|P_{j}-N_{j}\right| \leq \frac{1}{2 \pi}\|\nabla u\|_{L^{1}} ;
$$

this was proved by Brezis, Coron and Lieb [13].

By combining the above, a straightforward Cauchy sequences argument leads to the representation

$$
J u=\sum\left(\delta_{P_{j}}-\delta_{N_{j}}\right), \quad \text { with } P_{j}, N_{j} \in \overline{\mathbb{D}} \text { and } \sum\left|P_{j}-N_{j}\right| \leq \frac{1}{2 \pi}\|\nabla u\|_{L^{1}} ;
$$

this time, the sum may contain an infinite number of terms.

A remarkable fact is that there is a "converse" to (1.3): given sequences $\left(P_{j}\right),\left(N_{j}\right) \subset \overline{\mathbb{D}}$ such that $\sum_{j}\left|P_{j}-N_{j}\right|<\infty$, there exists some $u \in W^{1,1}\left(\mathbb{D} ; \mathbb{S}^{1}\right)$ such that $J u=\sum\left(\delta_{P_{j}}-\delta_{N_{j}}\right)$. The ingredients of the proof of this fact are already in [13], but the result was explicitly formulated much later; see [2] and also [16].

A similar approach can be followed for maps in $W^{1, k-1}\left(B^{k} ; \mathbb{S}^{k-1}\right), k \geq 2 .{ }^{4}$ However, for applications this functional setting is not sufficient. Indeed, the distributional Jacobian plays a crucial role e.g. in approximation problems [5, 34] or in the study of the Ginzburg-Landau equation [26,9]. In these contexts, the natural functional setting need not be the one of $W^{1, k-1}\left(B^{k} ; \mathbb{S}^{k-1}\right)$. For example, the trace space $W^{1 / 2,2}\left(\mathbb{S}^{2} ; \mathbb{S}^{1}\right)$ is related to the study of the Ginzburg-Landau equation [9]. The above suggests the following general program in the Sobolev space $X=W^{s, p}\left(\Omega ; \mathbb{S}^{k-1}\right)$, with $s>0,1 \leq p<\infty$ and $\Omega$ an open set in $\mathbb{R}^{m}$ or an $m$ dimensional manifold:

Task 1. Prove density in $X$ of a suitable class $\mathscr{R}$ of $\mathbb{S}^{k-1}$-valued maps $u$ smooth except on a small set $A(u)$.

\footnotetext{
${ }^{1}$ Here, $\wedge$ stands for the vector product of complex numbers: $a \wedge b=a_{1} b_{2}-a_{2} b_{1}$.

${ }^{2}$ Here, $\delta_{0}$ is the Dirac mass at the origin.

${ }^{3} \mathrm{By}$ invariance of the degree by homotopy, this integer is independent of the circle.

${ }^{4} \mathrm{Here}, B^{k}$ is the open unit ball in $\mathbb{R}^{k}$. The closed unit ball is denoted $\bar{B}^{k}$.
} 
Task 2. Define (possibly by density starting from maps in $\mathscr{R}$ ) the distributional Jacobian $J u$ of a map $u \in X$.

Task 3. Determine the range of the map $X \ni u \mapsto J u$.

Our paper presents new results concerning Tasks 2 and 3. Additional directions, though not discussed in the present paper, are equally interesting. An example: Bethuel [5] proved that the equality $J u=0$ characterizes the "absence of singularities": a map $u \in W^{1,2}\left(B^{3} ; \mathbb{S}^{2}\right)$ satisfies $J u=0$ if and only if $u$ is in the strong $W^{1,2}$-closure of smooth $\mathbb{S}^{2}$-maps; see also [7, 11,35]. The generalization of this result to different Sobolev regularities has not been obtained in full generality.

The above program has not been completely realized; we present below the state-of-the-art in these directions. To start with, let us define properly the class $\mathscr{R}$. From the pioneering work of Bethuel [6], the good candidate is known to be the class

$$
\mathscr{R}=\left\{u \in W^{s, p}\left(\Omega ; \mathbb{S}^{k-1}\right) \cap C^{\infty}(\Omega \backslash A(u)) ; \operatorname{dim} A(u)=m-[s p]-1\right\},
$$

where $A(u)$ is a finite union of Lipschitz manifolds (depending on $u$ ). Bethuel [6] proved density of $\mathscr{R}$ when $s=1$ and the target is an arbitrary compact oriented manifold. ${ }^{5}$ Very recently, Ponce, Van Schaftingen and the first author [12] extended this result for $s=2,3, \ldots$ On the other hand, density of $\mathscr{R}$ (for arbitrary targets) when $s<1$ was obtained by Brezis and the second author [15]. Another essentially known case is the one where $k-1 \leq s p<k$. This case can be tackled by adapting the projection method of Federer and Fleming [20]. The idea of using this method for manifold-valued maps goes back to Hardt, Kinderlehrer and Lin [23]. The projection method was used to prove density of the class $\mathscr{R}$ in the following cases:

a) When $s=1, k-1 \leq p<k$ in [8].

b) When $s=1 / 2, p=2, k=1, m=2$ in [36].

c) When $s<1$ and $k-1 \leq s p<k$ in [9]. ${ }^{6}$

d) When $s \geq 1,1 \leq s p<2$ and $k=2$ in [11].

Though the case where $k-1 \leq s p<k$ does not appear anywhere in full generality, the subcase $s=1$ is already in [8], the subcase $s<1$ is contained in [15], and the subcase $s>1$ is very likely to follow from the arguments in [11]. However, we emphasize the fact that, even though this special case is under control and even if the case of spheres is simpler than the one of arbitrary compact targets, density of $\mathscr{R}$ for sphere-valued maps and for arbitrary $m, k, s$ and $p$ is not known.

Task 2 concerns the possibility of defining $J u$. Some cases are to be discarded from the beginning:

a) When $s p<k-1$, smooth maps are dense in $W^{s, p}\left(\Omega ; \mathbb{S}^{k-1}\right)$ [28]. In this case, it is natural to let $J u=0$ for each $u$.

b) Same holds when $s p \geq m=\operatorname{dim} \Omega$. This goes back essentially to [37]; see also [17].

By the above, we may always assume that

$$
k-1 \leq s p<m,
$$

and we see that the limiting cases are the ones where $s p=k-1$. When $m=k, s=1$ and $p=k-1$, the distributional Jacobian $J u$ is defined as the divergence of an $L^{1}$-vector field:

$$
J u=\operatorname{div} \frac{1}{k \omega_{k}}\left(\operatorname{det}\left(u, \partial_{2} u, \ldots, \partial_{k} u\right), \operatorname{det}\left(\partial_{1} u, u, \partial_{3} u, \ldots, \partial_{k} u\right), \ldots, \operatorname{det}\left(\partial_{1} u, \ldots, \partial_{k-1} u, u\right)\right) ;
$$

here, $\omega_{k}$ is the volume of $B^{k}$. This definition can be adapted to the case where $m>k, s=1$ and $p=k-1$ [3, 25, 2]. We explain the procedure e.g. when $\Omega=\mathbb{S}^{m} .7$ We consider the volume form

\footnotetext{
5 The case of sphere-valued maps was previously known for $k-1 \leq p<k$ [8].

${ }^{6}$ The proof there is for special values of $s$ and $p$, but works without changes in the general case.

${ }^{7}$ All our results are stated for $\Omega=\mathbb{S}^{m}$, but it will be transparent from the proofs that $\Omega$ could be any bounded open set in $\mathbb{R}^{m}$ or $m$-dimensional compact manifold.
} 
on $\mathbb{S}^{k-1}$,

$$
\omega_{0}:=\frac{1}{k \omega_{k}} \sum_{i=1}^{k}(-1)^{i-1} y_{i} d y_{1} \wedge \ldots \wedge \widehat{d y_{i}} \wedge \ldots \wedge d y_{k}
$$

(with the convention that ${ }^{\wedge}$ denotes a missing term). For every $u \in W^{1, k-1}\left(\mathbb{S}^{m} ; \mathbb{S}^{k-1}\right)$, we define the $(m-k)$-current $J u$ by its action on the space $W^{1, \infty}\left(\Lambda^{m-k} \mathbb{S}^{m}\right)$ of Lipschitz $(m-k)$-forms in $\mathbb{S}^{m}:$

$$
\langle J u, \zeta\rangle:=(-1)^{m-k+1} \int_{\mathbb{S}^{m}} d \zeta \wedge\left(u^{\sharp} \omega_{0}\right), \quad \forall \zeta \in W^{1, \infty}\left(\Lambda^{m-k} \mathbb{S}^{m}\right) .
$$

Here, $u^{\sharp} \omega_{0}$ stands for the pullback

$$
u^{\sharp} \omega_{0}=\frac{1}{k \omega_{k}} \sum_{i=1}^{k}(-1)^{i-1} u_{i} d u_{1} \wedge \ldots \wedge \widehat{d u_{i}} \wedge \ldots \wedge d u_{k} .
$$

The formula (1.7) is a generalization of (1.5) when $m>k$. It is clear that $J u$ is well-defined as an element of the dual of $W^{1, \infty}\left(\Lambda^{m-k} \mathbb{S}^{m}\right)$, and that the map

$$
W^{1, k-1}\left(\mathbb{S}^{m} ; \mathbb{S}^{k-1}\right) \ni u \mapsto J u \in\left(W^{1, \infty}\left(\Lambda^{m-k} \mathbb{S}^{m}\right)\right)^{*}
$$

is continuous. Similarly, the distribution $J u$ is continuous wrt $u$ in the space $W^{s, p}\left(\mathbb{S}^{m} ; \mathbb{S}^{k-1}\right)$ provided $s \geq 1$ and $s p \geq k-1$. This follows from the embedding $W^{s, p} \cap L^{\infty} \hookrightarrow W^{1, k-1}$, valid when $s \geq 1$ and $s p \geq k-1$.

Existence of $J u$ is less clear when $s<1$ and $s p \geq k-1$. A first result in this direction was obtained by Hang and Lin [22]. These authors were able to define $J u$ for $u \in W^{s, p}\left(\mathbb{S}^{k} ; \mathbb{S}^{k-1}\right)$ provided $s \geq 1-1 / k$ and $s p \geq k-1$. This result was completed in [10]; there, it is proved that there is a natural way of defining $J u$ in the spaces $W^{s, p}\left(\mathbb{S}^{k} ; \mathbb{S}^{k-1}\right)$ when $s<1$ and $s p=k-1$. This is obtained as follows: first, the class $W^{1, k-1} \cap W^{s, p}\left(\mathbb{S}^{k} ; \mathbb{S}^{k-1}\right)$ is dense in $W^{s, p}\left(\mathbb{S}^{k} ; \mathbb{S}^{k-1}\right){ }^{8}$ Next, the key step in [10] consists in proving that $u \mapsto J u$, initially defined for maps in $W^{1, k-1} \cap$ $W^{s, p}\left(\mathbb{S}^{k} ; \mathbb{S}^{k-1}\right)$, is continuous wrt the $W^{s, p}$-norm. This gives a (unique) natural notion of $J u$ for $u \in W^{s, p}\left(\mathbb{S}^{k} ; \mathbb{S}^{k-1}\right)$, provided $s p \geq k-1$. Our first result is a generalization of the above to higher dimensions $m$.

1.1 Theorem. There exists a (unique) map

$$
J: \bigcup_{\substack{0<s \leq 1 \\ p=(k-1) / s}} W^{s, p}\left(\mathbb{S}^{m} ; \mathbb{S}^{k-1}\right) \rightarrow\left(W^{1, \infty}\left(\Lambda^{m-k} \mathbb{S}^{m}\right)\right)^{*}
$$

such that:

1. For $u \in W^{1, k-1}\left(\mathbb{S}^{m} ; \mathbb{S}^{k-1}\right)$, Ju coincides with the distributional Jacobian defined by (1.7).

2. The restriction of $J$ to every $W^{s, p}\left(\mathbb{S}^{m} ; \mathbb{S}^{k-1}\right)$ with $0<s<1$ and $p=(k-1) / s$ is continuous.

3. In addition, for $s$ and $p$ as above the following estimate holds:

$$
|\langle J u, \zeta\rangle| \leq C_{p}|u|_{W^{s, p}}^{p}\|d \zeta\|_{L^{\infty}}
$$

Here ||$_{W^{s, p}}$ stands for the standard semi-norm in $W^{s, p}$; for the convenience of the reader, its formula will be recalled in Section 2.

Task 2 is completed.

We next turn to Task 3: characterize the set $\left\{J u ; u \in W^{s, p}\left(\Omega ; \mathbb{S}^{k-1}\right)\right\}$. This requires establishing the analog of (1.3) and of its "converse". A first hint is given by [13]. There, it is proved that

$$
\inf \left\{\int_{\mathbb{R}^{3}}|\nabla u|^{2} ; J u=\sum_{j=1}^{k}\left(\delta_{P_{j}}-\delta_{N_{j}}\right)\right\}=8 \pi \inf _{\sigma \in S_{k}} \sum_{j=1}^{k}\left|P_{j}-N_{\sigma(j)}\right|,
$$

\footnotetext{
${ }^{8}$ This is proved in the same way as the density of the class $\mathscr{R}$.
} 
the sum in the right-hand side of (1.10) being the length of the shortest curve connecting the $P_{j}$ 's to the $N_{j}$ 's. It was conjectured in [13] that when, say, $m=4$ and $k=3$, the minimal Dirichlet energy required to create a Jacobian supported by a simple closed curve $M \subset \mathbb{R}^{4}$ equals

$8 \pi \min \{\mathbf{M}(N) ; N$ is a $2-$ rectifiable current such that $\partial N=M\}$.

Here, $\mathbf{M}(N)$ stands for the mass of $N$. For polygonal lines, this conjecture ${ }^{9}$ has been confirmed by Almgren, Browder and Lieb [3]. Far reaching extensions of this result were obtained by Alberti, Baldo and Orlandi [2]. Their results are the following: on the one hand,

$$
\left\{J u ; u \in \dot{W}^{1, k-1}\left(\mathbb{R}^{m} ; \mathbb{S}^{k-1}\right)\right\}=\{\partial N ; N \text { is an }(m-k+1)-\text { dimensional rectifiable current }\} .
$$

Here, $\dot{W}^{1, k-1}$ stands for the homogeneous Sobolev space. On the other hand,

$$
\inf \left\{\int_{\mathbb{R}^{k}}|\nabla u|^{k} ; J u=M\right\} \sim \inf \{\mathbf{M}(N) ; N \text { is rectifiable and } \partial N=M\} .
$$

Very recently, by combining the arguments in [3] and [2], Molnar [31] improved the conclusion (1.12) to

$$
\inf \left\{\int_{\mathbb{R}^{k}}|\nabla u|^{k} ; J u=M\right\}=(k-1)^{(k-1) / 2} k \omega_{k} \inf \{\mathbf{M}(N) ; N \text { rectifiable, } \partial N=M\} .
$$

A crucial point in [13, 3, 2] is the dipole construction discovered in [13]. Let us give an example: for $\mathbb{S}^{1}$-valued maps in $\mathbb{R}^{2}$, a dipole with a positive singularity $P_{1}$ and a negative singularity $N_{1}$ is a map $u: \mathbb{R}^{2} \rightarrow \mathbb{S}^{1}$ such that $J u=\delta_{P_{1}}-\delta_{N_{1}}$ which is, in addition, constant outside an arbitrarily small set $B(u)$. For arbitrary $s$ and $p$, one cannot control both the $W^{s, p}$-semi-norm of $u$ and the size of $B(u)$. However, this is possible in $W^{1,1}$ : for every $\varepsilon>0$, it is possible to construct $u$ such that $\|\nabla u\|_{L^{1}} \leq 2 \pi\left|P_{1}-N_{1}\right|+\varepsilon$ and $|B(u)| \leq \varepsilon$. An inspection of the dipole construction in [13] leads to the conclusion that this construction can be adapted to the critical spaces $W^{s, p}\left(\Omega ; \mathbb{S}^{k-1}\right)$ characterized by the equality $s p=k-1$. Our next results extend the results in [2] to the full scale of critical spaces.

1.2 Theorem. Let $0<s \leq k-1$ and $p \geq 1$ be such that $s p=k-1$. Assume that $m \geq k$. Let $u \in W^{s, p}\left(\mathbb{S}^{m} ; \mathbb{S}^{k-1}\right)$. Then there exists an $(m-k+1)$-rectifiable current $N$ in $\mathbb{S}^{m}$ such that

1. $\partial N=J u$.

2. $\mathbf{M}(N) \leq C_{p}|u|_{W^{s, p}}^{p}$.

1.3 Theorem. Let $M$ be the boundary of an $(m-k+1)$-rectifiable current $N$ in $\mathbb{S}^{m}$. Then there exists $u \in \bigcap_{s p=k-1} W^{s, p}\left(\mathbb{S}^{m} ; \mathbb{S}^{k-1}\right)$ such that:

1. $J u=M$.

2. $|u|_{W^{s, p}}^{p} \leq C_{p} \mathbf{M}(N)$ if $s p=k-1$.

Let us discuss how Theorems 1.2 and 1.3 compare to the corresponding results in [2]. When $k \geq 3$, the critical spaces are nested: if the couples $\left(s_{1}, p_{1}\right)$ and $\left(s_{2}, p_{2}\right)$ are critical and $s_{1}>s_{2}$, then we have the Gagliardo-Nirenberg type embeddings $W^{s_{1}, p_{1}}\left(\mathbb{S}^{m} ; \mathbb{S}^{k-1}\right) \hookrightarrow W^{s_{2}, p_{2}}\left(\mathbb{S}^{m} ; \mathbb{S}^{k-1}\right)$. Therefore, Theorem 1.3 follows from the special case $s=k-1, p=1$ combined with the GagliardoNirenberg inequalities. In turn, we prove this special case by adapting the techniques in [2]. As explained in the course of the proof, the new technical difficulties ${ }^{10}$ arise from the fact that we need smooth (and not merely Lipschitz) dipoles and from the fact that we work on $\mathbb{S}^{m}$ instead of $\mathbb{R}^{m} \cdot{ }^{11}$ However, the case $k=2$ does not follow from the above strategy. This is due to the fact that the corresponding Gagliardo-Nirenberg embeddings are wrong: e.g., we do not have

\footnotetext{
${ }^{9}$ And its generalization to arbitrary $m$ and $k$.

${ }^{10}$ When compared to [2].

${ }^{11}$ Our proof adapts also to the case of a bounded domain in $\mathbb{R}^{m}$.
} 
$W^{1,1}\left(\mathbb{S}^{m} ; \mathbb{S}^{1}\right) \hookrightarrow W^{1 / 2,2}\left(\mathbb{S}^{m} ; \mathbb{S}^{1}\right)$. In this case, we present two proofs. One of them is "geometric": it relies on a dipole construction with control of several norms combined with a Cauchy sequences argument. The second one is purely "analytical": by an averaging argument reminiscent of the projection method of Federer and Fleming [20], we associate to each function $u$ a "better" function with the same singular set. ${ }^{12}$ We think that this second approach is of independent interest and may serve in other situations. The proof of Theorem 1.2 is very much in the spirit of Almgren, Browder and Lieb [3], who were the first to use the coarea formula in the proof of estimates for sphere-valued maps. Later, Hang and Lin [22] and Alberti, Baldo and Orlandi [2] used a similar approach in order to establish special cases of Theorem 1.2. Our approach is similar to the one in [22], but we had to face new technical difficulties. A final remark about the coarea formula setting. The content of Theorem 1.2 is that $J u$ is the boundary of a rectifiable current $N$. The main idea of [3] is to take $N=u^{-1}(a)$ for some appropriate $a \in \mathbb{S}^{k-1}$; this idea is also at the heart of [2]. This leads, in general, to a nonsmooth $N .{ }^{13}$ It turns out that it is possible to write $J u=\partial M$ with smooth $M$ provided we let $M$ live in the higher dimensional space $\mathbb{S}^{m} \times[0, \infty)$; this is how our construction goes. ${ }^{14}$ We next land on $\mathbb{S}^{m}$ by letting $N$ be the projection of $M$. Technically, this leads us to the use of the standard coarea formula, as opposed to its Sobolev spaces version required in [2].

Task 3 is thus completed in the scale of critical spaces. However, little is known about the non critical spaces. To give an example, the characterization of the space $\left\{J u ; u \in W^{1, p}\left(\mathbb{S}^{3} ; \mathbb{S}^{2}\right\}\right.$, with $2<p<3$, is not known. The situation is completely understood only for circle-valued maps. The space $\left\{J u ; u \in W^{s, p}\left(\mathbb{S}^{m} ; \mathbb{S}^{1}\right\}\right.$, with $1<s p<2$, has been characterized in [11] when $s \geq 1$ and in [30] when $s<1 .{ }^{15}$ However, the proofs there rely heavily on the fact that the target space is $\mathbb{S}^{1}$. The case where the target is $\mathbb{S}^{k-1}$ with $k \geq 3$ is widely open.

Our paper is organized as follows: in Sections 2, 3 and 4 we recall, for the convenience of the reader, the main analytical tools required in the proofs. In Section 5, we establish Theorems 1.1 and 1.2. The proof of Theorem 1.1 relies on a technical lemma, Lemma 5.1, whose long proof is postponed to Section 7. Section 6 is devoted to the proof of Theorem 1.3.

\section{Sobolev spaces of currents and forms}

Our presentation follows essentially [21, Chapters 2, 3]. Let $H$ be a subspace of the Euclidean space $\mathbb{R}^{m}, m \geq 1$. For $0 \leq l$, we denote by $\Lambda_{l} H$ the vector space of $l$-vectors in $H$ and by $\Lambda^{l} H=$ $\Lambda_{l} H^{*}$ the vector space of $l$-covectors in $H$. The duality pairing between $\Lambda_{l} H$ and $\Lambda^{l} H$ is defined in terms of simple vectors and covectors as follows: If $h=h_{1} \wedge \ldots \wedge h_{l} \in \Lambda_{l} H, h_{j} \in H$ and $\theta=\theta_{1} \wedge \ldots \theta_{l} \in \Lambda^{l} H, \theta_{i} \in H^{*}$, then

$$
\langle h, \theta\rangle=\langle\theta, h\rangle=\operatorname{det}\left(\theta_{i}\left(h_{j}\right)\right) .
$$

The inner product of vectors and covectors (which only depends on the inner product defined on $\left.\mathbb{R}^{m}\right)$ is defined as follows. Let $\left(e_{i}\right)$ denote an orthonormal basis in $\mathbb{R}^{m}, e_{i}^{*}$ be the dual basis, and set

$$
I(l, m)=\left\{\left(i_{1}, \ldots, i_{l}\right) ; 1 \leq i_{1}<\ldots<i_{l} \leq m\right\} .
$$

If we set

$$
e_{I}=e_{i_{1}} \wedge \ldots \wedge e_{i_{l}}, e_{I}^{*}=e_{i_{1}}^{*} \wedge \ldots \wedge e_{i_{l}}^{*}, \quad \forall I \in I(l, m),
$$

then, for $\xi=\sum_{I} \xi_{I} e_{I}, \xi^{\prime}=\sum_{I} \xi_{I}^{\prime} e_{I}, \alpha=\sum_{I} \alpha_{I} e_{I}^{*}, \alpha^{\prime}=\sum_{I} \alpha_{I}^{\prime} e_{I}^{*}$, we define

$$
\left\langle\xi, \xi^{\prime}\right\rangle=\sum_{I} \xi_{I} \xi_{I}^{\prime},\left\langle\alpha, \alpha^{\prime}\right\rangle=\sum_{I} \alpha_{I} \alpha_{I}^{\prime},
$$

\footnotetext{
${ }^{12}$ The key result is Lemma 6.15 .

${ }^{13}$ This is due to the fact that $J u$ need not be the boundary of a smooth $N$. See the discussion in [2, p. 277].

${ }^{14}$ This approach is inspired by [22].

${ }^{15}$ The results in [30] are described in [29].
} 


$$
|\xi|=\left(\sum_{I}\left|\xi_{I}\right|^{2}\right)^{1 / 2},|\alpha|=\left(\sum_{I}\left|\alpha_{I}\right|^{2}\right)^{1 / 2} .
$$

The above quantities do not depend on the choice of the orthonormal basis $\left(e_{i}\right)$.

A linear map $L: H \rightarrow K$ induces the morphism

$$
\Lambda_{l} L: \Lambda_{l} H \rightarrow \Lambda_{l} K, \Lambda_{l} H \ni v_{1} \wedge \ldots \wedge v_{l} \stackrel{\Lambda_{l} L}{\longrightarrow} L v_{1} \wedge \ldots \wedge L v_{l} \in \Lambda_{l} K
$$

and its adjoint $\Lambda^{l} L: \Lambda^{l} K \rightarrow \Lambda^{l} H$.

Let $\Omega \subset \mathbb{R}^{m}$ be an open set. For $0 \leq l \leq m$, we denote by $C^{\infty}\left(\Lambda^{l} \Omega\right.$ ) (an abbreviation for $C^{\infty}\left(\Omega ; \Lambda^{l} \mathbb{R}^{m}\right)$ ) the set of smooth $l$-forms in $\Omega$. Similarly, $C_{c}^{\infty}\left(\Lambda^{l} \Omega\right)$ is the set of smooth $l$-forms compactly supported in $\Omega$. The differential of a form $\omega$ is denoted by $d \omega$. An $l$-current $T \in$ $\left(C_{c}^{\infty}\left(\Lambda^{l} \Omega\right)\right)^{*}, l \geq 0$, is a continuous linear form on the set $C_{c}^{\infty}\left(\Lambda^{l} \Omega\right)$ endowed with the topology of distributions. The boundary of an $l$-current $T, l \geq 1$, is the $(l-1)$-current $\partial T \in\left(C_{c}^{\infty}\left(\Lambda^{l-1} \Omega\right)\right)^{*}$ defined by

$$
\langle\partial T, \omega\rangle=\langle T, d \omega\rangle, \quad \forall \omega \in C_{c}^{\infty}\left(\Lambda^{l-1} \Omega\right) .
$$

For $0<s<\infty, 1 \leq p<\infty$, we denote by $W^{s, p}\left(\Lambda^{l} \Omega\right)$ the set of $l$-forms such that

$$
\|\omega\|_{W^{s, p}}:=\sum_{k \leq[s]}\left\|D^{k} \omega\right\|_{L^{p}}+\left|D^{[s]} \omega\right|_{W^{\sigma, p}}<\infty
$$

where $[s]$ is the integer part of $s, \sigma:=s-[s]$ and for $\omega=\sum \omega_{I} d x_{I}, d x_{I}=d x_{i_{1}} \wedge \ldots \wedge d x_{i_{l}}$,

$$
\begin{aligned}
& \left\|D^{k} \omega\right\|_{L^{p}}:=\left\{\sum_{I} \sum_{|\alpha|=k} \int_{\Omega}\left|\frac{\partial^{\alpha} \omega_{I}}{\partial x_{\alpha}}(x)\right|^{p} d x\right\}^{1 / p} \\
& \left|D^{[s]} \omega\right|_{W^{\sigma, p}}:=\left\{\sum_{I|\alpha|=[s]} \int_{\Omega} \int_{\Omega} \frac{\left|\frac{\partial^{\alpha} \omega_{I}}{\partial x_{\alpha}}(x)-\frac{\partial^{\alpha} \omega_{I}}{\partial x_{\alpha}}(y)\right|^{p}}{|x-y|^{m+\sigma p}} d x d y\right\}^{1 / p} .
\end{aligned}
$$

The definition extends, with the obvious modification, to the case $p=\infty$.

We next turn to the definition of Sobolev spaces of forms on manifolds. This requires defining the pullback of forms. Let $\varphi: \Omega_{1} \subset \mathbb{R}^{m_{1}} \rightarrow \Omega_{2} \subset \mathbb{R}^{m_{2}}$ be a smooth map between two open sets $\Omega_{1}$ and $\Omega_{2}$. The pullback $\varphi^{\sharp} \omega$ of a measurable $l$-form $\omega$ in $\Omega_{2}$ is defined by

$$
\left(\varphi^{\sharp} \omega\right)(x)\left(\eta_{1}, \ldots, \eta_{l}\right)=\omega(\varphi(x))\left(d \varphi(x)\left(\eta_{1}\right), \ldots, d \varphi(x)\left(\eta_{l}\right)\right), \quad \forall x \in \Omega_{1}, \eta_{1}, \ldots, \eta_{l} \in \mathbb{R}^{m_{1}} .
$$

Then $\varphi^{\sharp} \omega$ is a measurable $l$-form on $\Omega_{1}$, and, if $\omega$ is smooth, then we have the chain rule $d \varphi^{\sharp} \omega=\varphi^{\sharp} d \omega$.

Let $\varphi: \Omega_{1} \subset \mathbb{R}^{m_{1}} \rightarrow \Omega_{2} \subset \mathbb{R}^{m_{2}}$ be a smooth proper map between two open sets $\Omega_{1}$ and $\Omega_{2}$. Then we define the pushforward $\varphi_{\sharp} T$ of an $l$-current $T \in\left(C_{c}^{\infty}\left(\Lambda^{l} \Omega_{1}\right)\right)^{*}$ as the following $l$-current on $\Omega_{2}$ :

$$
\left\langle\varphi_{\sharp} T, \omega\right\rangle=\left\langle T, \varphi^{\sharp} \omega\right\rangle, \quad \forall \omega \in C_{c}^{\infty}\left(\Lambda^{l} \Omega_{2}\right) .
$$

In the special case where $\varphi: \Omega_{1} \rightarrow \Omega_{2}$ is a diffeomorphism, we may define two additional objects: $\varphi_{\sharp} \omega=\left(\varphi^{-1}\right)^{\sharp} \omega$ and $\varphi^{\sharp} T=\left(\varphi^{-1}\right)_{\sharp} T$, whenever $\omega$ is a form on $\Omega_{1}$ and $T$ is a current on $\Omega_{2}$.

We are now in position to define the Sobolev spaces of forms on manifolds. Consider a smooth oriented compact $m$-dimensional boundaryless submanifold $M$ of $\mathbb{R}^{m+n}$. The set $\Lambda^{l} M:=\bigcup_{x \in M}\left(\{x\} \times \Lambda^{l} T_{x} M\right)$ has a natural differential structure. Same for $\Lambda_{l} M:=\bigcup_{x \in M}\left(\{x\} \times \Lambda_{l} T_{x} M\right)$.

The set of smooth compactly supported $l$-forms in $M$ is denoted by $C_{c}^{\infty}\left(\Lambda^{l} M\right)$. This set reduces to the set $C^{\infty}\left(\Lambda^{l} M\right)$ of smooth $l$-forms on $M$ when $M$ is compact. In order to simplify the presentation, we further assume that either $M$ is compact, or $M=N \times \omega$, with $N$ compact and $t$-dimensional and $\omega \subset \mathbb{R}^{l}$ (this will indeed be the case of all the submanifolds considered later). The definition of $W^{s, p}\left(\Lambda^{l} M\right)$ follows from that of $W^{s, p}\left(\Lambda^{l} \Omega\right)$ via a finite covering of $M$ by domains of local coordinates. Let $\left(\varphi_{j}, U_{j}, V_{j}\right)$ be a finite atlas of $M$, where: 
a) If $M$ is compact, then $\varphi_{j}: \overline{U_{j}} \subset M \rightarrow \overline{V_{j}} \subset \mathbb{R}^{m}$ is a smooth diffeomorphism.

b) If $M=N \times \omega$, then $\varphi_{j}=\eta_{j} \otimes$ Id, with $\eta_{j}: \overline{W_{j}} \subset N \rightarrow \overline{X_{j}} \subset \mathbb{R}^{t}$ is a smooth diffeomorphism. Here, $\left(W_{j}\right)$ is a covering of $N$ with domains of local coordinates.

Consider maps $\theta_{j}: M \rightarrow[0,1]$, such that:

a) If $M$ is compact, then $\left(\theta_{j}\right)$ is a partition of the unit subordinated to the covering $\left(U_{j}\right)$.

b) If $M=N \times \omega$, then $\theta_{j}=\psi_{j} \otimes \mathrm{Id}$, with $\left(\psi_{j}\right)$ partition of the unit subordinated to the covering $\left(W_{j}\right)$ of $N$.

For $s \geq 0$ and $p \geq 1$, we let

$$
\|\omega\|_{W^{s, p}}=\|\omega\|_{W^{s, p}\left(\Lambda^{l} M\right)}=\sum_{j}\left\|\varphi_{j \sharp}\left(\theta_{j} \omega\right)\right\|_{W^{s, p}\left(\Lambda^{l} V_{j}\right)} .
$$

Two different choices of finite coverings lead to the same space $W^{s, p}\left(\Lambda^{l} M\right)$, with equivalent norms.

Let $0<s$ and $1 \leq p \leq \infty$. When $\omega \in W_{l o c}^{s, p}\left(\Lambda^{0} M\right),{ }^{16}$ we define the seminorm $|\omega|_{W^{s, p}(M)} \in[0, \infty]$ as follows.

$$
|\omega|_{W^{s, p}(M)}^{p}=\left\{\begin{array}{ll}
\int_{M} \int_{M} \frac{|\omega(x)-\omega(y)|^{p}}{d(x, y)^{m+s p}} d x d y, & \text { if } s<1 \\
\int_{M}|d \omega|^{p}, & \text { if } s=1 \\
\|d \omega\|_{W^{s-1, p}}, & \text { if } s>1
\end{array},\right.
$$

with the obvious modification when $p=\infty$. Here, $d(x, y)$ is the geodesic distance between $x$ and $y$.

An equivalent semi-norm is given by $\left\|\omega-f_{M} \omega\right\|_{W^{s, p}}$. Alternatively, one can consider the standard norm on the quotient space $W^{s, p} / \mathbb{R} .{ }^{17}$ Other (equivalent) semi-norms will be introduced in the next sections.

We next identify currents defined in $M$ with currents defined in $\mathbb{R}^{m+n}$. This goes as follows. If $\varphi$ is a smooth map defined on $M$ with values into some other manifold, then one may define the pullback and the pushforward by $\varphi$ as above. Let $U$ be an open neighborhood of $M$ in $\mathbb{R}^{m+n}$ and

$$
i: M \rightarrow U, M \ni \underset{i}{\rightarrow} x \in U
$$

be the canonical inclusion. Given an $l$-form $\omega$ on $U$, we denote by $\omega_{\mid M}$ the form on $M$ defined by $i^{\sharp} \omega$. We say that

1. A form $\omega \in C_{c}^{\infty}\left(\Lambda^{l} U\right)$ is null at $M$ if $i^{\sharp} \omega=0$,

2. An $l$-current $T \in\left(C_{c}^{\infty}\left(\Lambda^{l} U\right)\right)^{*}$ is a current along $M$ if $\langle T, \omega\rangle=0$ for every $\omega \in C_{c}^{\infty}\left(\Lambda^{l} U\right)$ which is null at $M$.

One can easily see that $T \mapsto i_{\sharp} T$ is an isomorphism between the space of $l$-currents defined in $M$ and the space of $l$-currents along $M .^{18}$

\section{Rectifiability, area and coarea}

We follow [19, Chapters 2, 3]; see also [21, Chapter 2] and [32, Chapters 3, 4].

An $m$-rectifiable set $\mathscr{M} \subset \mathbb{R}^{m+n}$ is a set of the form $\mathscr{M}=\mathscr{M}_{0} \cup \bigsqcup_{j=1}^{\infty} \mathscr{M}_{j}$, where

1. $\sqcup$ stands for a disjoint union.

\footnotetext{
${ }^{16}$ In this case, $\omega$ is identified to a function.

${ }^{17}$ We will apply this with $\omega$ an $\mathbb{R}^{k}$-valued map. In this case, the quotient space is $\left(W^{s, p}\left(\Lambda^{0} M\right)\right)^{k} / \mathbb{R}^{k}$.

${ }^{18}$ Here, the spaces of currents are endowed with the usual topologies.
} 
2. $\mathscr{H}^{m}\left(\mathscr{M}_{0}\right)=0$.

3. For $j \geq 1$, every $\mathscr{M}_{j}$ is a Borel subset of an $m$-dimensional $C^{1}$-submanifold $\mathscr{N}_{j}$ of $\mathbb{R}^{m+n}$.

4. The total measure $\mathscr{H}^{m}(\mathscr{M})=\sum_{j=1}^{\infty} \mathscr{H}^{m}\left(\mathscr{M}_{j}\right)$ is finite.

Let $\mathscr{M}$ be $m$-rectifiable. An $m$-dimensional subspace $P$ of $\mathbb{R}^{m+n}$ is the approximate tangent space of $\mathscr{M}$ at $x \in \mathbb{R}^{m+n}$ if

$$
\lim _{\lambda \rightarrow 0^{+}} \int_{(\mathscr{M}-x) / \lambda} g(y) d \mathscr{H}^{m}(y)=\int_{P} g(y) d \mathscr{H}^{m}(y), \quad \forall g \in C_{c}^{\infty}\left(\mathbb{R}^{m+n}\right) .
$$

If it exists, such a $P$ is unique and is denoted by $T_{x} \mathscr{M}$. An $m$-rectifiable set $\mathscr{M}$ admits $\mathscr{H}^{m}$-a.e. on $\mathcal{M}$ an approximate tangent space [21, Section 2.1.4], and moreover

$$
T_{x} \mathscr{M}=T_{x} \mathscr{N}_{j}, \quad \mathscr{H}^{m}-\text { a.e. } x \in \mathcal{M}_{j} .
$$

An $m$-rectifiable current ${ }^{19}$ in the open set $U \subset \mathbb{R}^{m+n}$ is defined via a triple $(\mathscr{M}, \xi, \theta)$, where

1. $\mathscr{M} \subset U$ is an $m$-rectifiable set.

2. $\xi: \mathscr{M} \rightarrow \Lambda_{m} \mathbb{R}^{m+n}$ is a Borel measurable map such that $|\xi|=1$ and $\xi(x) \in \Lambda_{m} T_{x} \mathscr{M}$ for $\mathscr{H}^{m}$-a.e. $x .^{20}$

3. $\theta: \mathscr{M} \rightarrow \mathbb{Z}$ is a Borel locally $\mathscr{H}^{m}$-summable function. ${ }^{21}$

The $m$-rectifiable current associated to this triple acts through the formula

$$
\langle T, \omega\rangle=\int_{\mathscr{M}}\langle\xi, \omega\rangle \theta d \mathscr{H}^{m}, \quad \forall \omega \in C_{c}^{\infty}\left(\Lambda^{m} U\right) .
$$

Clearly, such a $T$ acts on the space of compactly supported $m$-forms with continuous coefficients, and thus it may be identified with a (vector-valued) measure. As a measure, the total variation (mass) of $T$ is given by

$$
\mathbf{M}(T):=\int_{\mathscr{M}}|\theta| d \mathscr{H}^{m} .
$$

We end this section by recalling the area and coarea formulae.

Let $M$ be a smooth $m$-dimensional submanifold of an Euclidean space. Let $f \in \operatorname{Lip} p_{l o c}\left(M ; \mathbb{R}^{k}\right)$. We define the Jacobian of $f$ at $x \in M$ by

$$
J_{f}^{M}(x):=\left\{\begin{array}{ll}
\left(\operatorname{det}(d f(x))^{*}(d f(x))\right)^{1 / 2}, & \text { if } k \geq m \\
\left(\operatorname{det}(d f(x))(d f(x))^{*}\right)^{1 / 2}, & \text { if } k \leq m
\end{array} .\right.
$$

Here, the differential $d f(x): T_{x} M \rightarrow \mathbb{R}^{k}$ of $f$ at $x$ is defined for a.e. $x$. The area formula and coarea formula read as follows [21, Section 2.1.5]. Let $f: M \rightarrow \mathbb{R}^{k}$ be locally Lipschitz. Let $A \subset M$ be an $\mathscr{H}^{m}$-measurable set. Then for any nonnegative Borel measurable function $u: M \rightarrow \mathbb{R}$, we have

1. (Area formula) If $k \geq m$, then

$$
\int_{A} u(x) J_{f}^{M}(x) d \mathscr{H}^{m}(x)=\int_{f(A)}\left(\sum_{f^{-1}(y) \cap A} u(x)\right) d \mathscr{H}^{m}(y) .
$$

2. (Coarea formula) If $k<m$, then

$$
\int_{A} u(x) J_{f}^{M}(x) d \mathscr{H}^{m}(x)=\int_{f(A)}\left(\int_{f^{-1}(y) \cap A} u d \mathscr{H}^{m-k}(x)\right) d \mathscr{H}^{k}(y) .
$$

${ }^{19}$ Sometimes refered as an integer multiplicity $m$-rectifiable current.

$20 \xi$ is a Borel measurable orientation.

${ }^{21} \theta$ is the multiplicity function. By changing $\xi$ if necessary, one may assume that $\theta$ takes only nonnegative values. 


\section{Interpolation inequalities}

We start by recalling the following Gagliardo-Nirenberg type inequality [33, Lecture II], [14, Corollary 2]. ${ }^{22}$

4.1 Lemma. Let $\theta \in(0,1), a>0, p \geq 1,1<q \leq \infty$. Let $r$ satisfy $\frac{1}{r}=\frac{\theta}{p}+\frac{1-\theta}{q}$. The inequality

$$
\|u\|_{W^{\theta a, r}} \leq C\|u\|_{W^{a, p}}^{\theta}\|u\|_{L^{q}}^{1-\theta}, \quad \forall u \in C_{c}^{\infty}\left(\mathbb{R}^{m}\right)
$$

is true in the following cases.

1. a is not an integer.

2. $a$ and $\theta a$ are integers.

3. $a$ is an integer and $p>1$.

As a consequence, we have

4.2 Lemma. For every $p \geq 1$ and $k \geq 3$, there exists $C=C(p, m)>0$ such that

$$
\|u\|_{W^{(k-1) / p, p}\left(\mathbb{S}^{m}\right)} \leq C\|u\|_{W^{k-1,1}\left(\mathbb{S}^{m}\right)}^{1 / p}\|u\|_{L^{\infty}\left(\mathbb{S}^{m}\right)}^{1-1 / p}, \quad \forall u \in C^{\infty}\left(\mathbb{S}^{m}\right) .
$$

Proof. It suffices to prove (4.2) for $u \in C_{c}^{\infty}\left(B^{m}\right)$. Indeed, assume that (4.2) holds in this case. Consider a covering of $\mathbb{S}^{m}$ with finitely many open subsets $U_{j}$ such that $\overline{U_{j}}$ is diffeomorphic to the closed ball $\bar{B}^{m}$. We denote by $\varphi_{j}: \overline{U_{j}} \rightarrow \bar{B}^{m}$ the corresponding diffeomorphisms, and by $\left(\theta_{j}\right)$ a partition of the unit subordinated to the covering $\left(U_{j}\right)$. Then, for every $u \in C^{\infty}\left(\mathbb{S}^{m}\right)$ we have

$$
\begin{aligned}
\|u\|_{W^{(k-1) / p, p}\left(\mathbb{S}^{m}\right)} & \sim \sum_{j}\left\|\left(\varphi_{j}\right)_{\sharp}\left(\theta_{j} u\right)\right\|_{W^{(k-1) / p, p}\left(B^{m}\right)} \leq C \sum_{j}\left\|\left(\varphi_{j}\right)_{\sharp}\left(\theta_{j} u\right)\right\|_{W^{k-1,1}\left(B^{m}\right)}^{1 / p}\left\|\left(\varphi_{j}\right)_{\sharp}\left(\theta_{j} u\right)\right\|_{L^{\infty}\left(B^{m}\right)}^{1-1 / p} \\
& \leq C\|u\|_{L^{\infty}\left(\mathbb{S}^{m}\right)}^{1-1 / p} \sum_{j}\left\|\left(\varphi_{j}\right)_{\sharp}\left(\theta_{j} u\right)\right\|_{W^{k-1,1}\left(B^{m}\right)}^{1 / p} \leq C\|u\|_{L^{\infty}\left(\mathbb{S}^{m}\right)}^{1-1 / p}\|u\|_{W^{k-1,1}\left(\mathbb{S}^{m}\right)}^{1 / p},
\end{aligned}
$$

as claimed.

Let $u \in C_{c}^{\infty}\left(B^{m}\right)$. Since (4.2) is trivially true for $p=1$, we may assume that $p>1$. By item 2 in Lemma 4.1, we have

$$
\|u\|_{W^{j,(k-1) / j}} \leq C\|u\|_{W^{k-1,1}}^{j /(k-1)}\|u\|_{L^{\infty}}^{1-j /(k-1)}, \quad \forall j \in \llbracket 1, \ldots, k-1 \rrbracket .
$$

We claim that (4.2) is a consequence of the following inequality:

$$
\|u\|_{W^{s, p}} \leq C\|u\|_{W^{1,1}}^{s}\|u\|_{L^{q}}^{1-s}, \quad \forall s \in(0,1), q \in(1, \infty), u \in C_{c}^{\infty}\left(\mathbb{R}^{m}\right),
$$

where $p$ is defined by $\frac{1}{p}=s+\frac{1-s}{q}$. Indeed, assume for a moment that (4.4) is true. Then we are able to complete the proof of (4.2) by considering three different cases.

Case 1. $\frac{k-1}{p} \in(k-2, k-1)$.

By (4.3), we have

$$
\|u\|_{W^{k-2,(k-1)(k-2)}} \leq C\|u\|_{W^{k-1,1}}^{(k-2) /(k-1)}\|u\|_{L^{\infty}}^{1-(k-2) /(k-1)} .
$$

By combining this estimate with (4.4) applied to the map $D^{k-2} u$, to $q=\frac{k-1}{k-2}$ and to $s=\frac{k-1}{p}-$ $(k-2)$, we obtain

$$
\begin{aligned}
\left\|D^{k-2} u\right\|_{W^{(k-1) / p-(k-2), p}} & \leq C\left\|D^{k-2} u\right\|_{W^{1,1}}^{(k-1) / p-(k-2)}\left\|D^{k-2} u\right\|_{L^{(k-1) /(k-2)}}^{1-(k-1) / p+k-2} \leq(\text { by }(4.5)) \\
& \leq C\left\|D^{k-2} u\right\|_{W^{1,1}}^{(k-1) / p-(k-2)}\left(\|u\|_{W^{k-1,1}}^{(k-2) /(k-1)}\|u\|_{L^{\infty}}^{1 /(k-1)}\right)^{1-(k-1) / p+k-2} \\
& \leq C\|u\|_{W^{k-1,1}}^{1 / p}\|u\|_{L^{\infty}}^{1-1 / p} .
\end{aligned}
$$

\footnotetext{
${ }^{22}$ The results in [14] are stated only for $p>1$, but the arguments still hold when $p=1$.
} 
Using Rellich's theorem, one may prove by contradiction that there exists some $C>0$ such that the following Poincaré type inequality holds:

$$
\|u\|_{W^{(k-1) / p, p}} \leq C\left\|D^{k-2} u\right\|_{W^{(k-1) / p-k+2, p}}, \quad \forall u \in C_{c}^{\infty}\left(B^{m}\right) .
$$

We obtain (4.2) by combining (4.6) and (4.7).

Case 2. $\frac{k-1}{p}$ is an integer.

This case follows from item 2 in Lemma 4.1.

Case 3. $\frac{k-1}{p} \in(l-1, l)$ for some integer $l \in \llbracket 1, k-2 \rrbracket$.

In this case, we have

$$
\begin{aligned}
\|u\|_{W^{(k-1) / p, p}} & \leq C\|u\|_{W^{l,(k-1) / l}}^{(k-1) /(p l)}\|u\|_{L^{\infty}}^{1-(k-1) /(p l)} \quad(\text { by }(4.1)) \\
& \leq C\left(\|u\|_{W^{k-1,1}}^{l /(k-1)}\|u\|_{L^{\infty}}^{1-l /(k-1)}\right)^{(k-1) /(p l)}\|u\|_{L^{\infty}}^{1-(k-1) /(p l)} \quad \text { (by (4.3)) } \\
& \leq C\|u\|_{W^{k-1,1}}^{1 / p}\|u\|_{L^{\infty}}^{1-1 / p} ;
\end{aligned}
$$

that is (4.2) holds.

It remains to prove (4.4). Let $\varepsilon \in(0, s)$. By [18, Theorem 1.5], we have

$$
\|u\|_{W^{s, p}} \leq C\|u\|_{W^{1,1}}^{\theta_{\varepsilon}}\|u\|_{W^{\varepsilon, r}}^{1-\theta_{\varepsilon}},
$$

where $\theta_{\varepsilon}=\frac{s-\varepsilon}{1-\varepsilon}$ and $r$ is defined by $\frac{1}{p}=\theta_{\varepsilon}+\frac{1-\theta_{\varepsilon}}{r}$.

By (4.1), we have

$$
\|u\|_{W^{\varepsilon, r}} \leq C\|u\|_{L^{q}}^{\eta_{\varepsilon}}\|u\|_{W^{s, p}}^{1-\eta_{\varepsilon}},
$$

where $\eta_{\varepsilon}=1-\frac{\varepsilon}{s}$ satisfies $\frac{1}{r}=\frac{\eta_{\varepsilon}}{q}+\frac{1-\eta_{\varepsilon}}{p}$.

We obtain (4.2) by combining (4.8) with (4.9). The proof of Lemma 4.2 is complete.

4.3 Corollary. For every $p \geq 1$ and $k \geq 3$, there exists $C=C(p, m)>0$ such that

$$
|u|_{W^{(k-1) / p, p}\left(\mathbb{S}^{m}\right)} \leq C|u|_{W^{k-1,1}\left(\mathbb{S}^{m}\right)}^{1 / p}\|u\|_{L^{\infty}\left(\mathbb{S}^{m}\right)}^{1-1 / p}, \quad \forall u \in C^{\infty}\left(\mathbb{S}^{m}\right) .
$$

Proof. Apply Lemma 4.2 to $u-f_{\mathbb{S}^{m}} u$.

4.4 Remark. In the proof of Theorem 1.2, item 2 , it suffices to consider the case where $p>k-1$. Indeed, assume that the conclusion of Theorem 1.2 holds when $p>k-1$. Let $u \in W^{(k-1) / p, p}\left(\mathbb{S}^{m} ; \mathbb{S}^{k-1}\right)$ for some $p \leq k-1$.

Assume first that $p>1$. Let $s \in(0,1)$. By Lemma 4.1, we have $u \in W^{s,(k-1) / s}\left(\mathbb{S}^{m} ; \mathbb{S}^{k-1}\right)$ and the argument leading to Corollary 4.3 yields

$$
|u|_{W^{s,(k-1) / s}\left(\mathbb{S}^{m}\right)} \leq C|u|_{W^{(k-1) / p, p}\left(\mathbb{S}^{m}\right)}^{(s p) /((k-1)}\|u\|_{L^{\infty}\left(\mathbb{S}^{m}\right)}^{1-(k p) /(k-1)} \text {. }
$$

By Theorem 1.2, there exists some $(m-k+1)$-rectifiable current $N$ in $\mathbb{S}^{m}$ such that $J u=\partial N$ and

$$
\mathbf{M}(N) \leq C|u|_{W^{s,(k-1) / s}\left(\mathbb{S}^{m}\right)}^{(k-1) / s} \leq C|u|_{W^{(k-1) / p, p}\left(\mathbb{S}^{m}\right)}^{p} .
$$

When $p=1$, Lemma 4.1 combined with the argument leading to Corollary 4.3 implies

$$
|u|_{W^{1, k-1}\left(\mathbb{S}^{m}\right)} \leq C|u|_{W^{k-1,1}\left(\mathbb{S}^{m}\right)}^{1 /(k-1)}\|u\|_{L^{\infty}\left(\mathbb{S}^{m}\right)}^{1-1 /(k-1)} .
$$

In this case, it suffices to invoke Theorem 1.2 in the space $W^{1, k-1}\left(\Omega ; \mathbb{S}^{k-1}\right)$, which was proved in [2, Theorem 3.8]. Here, $\Omega \subset \mathbb{R}^{m}$ is an open set, and not $\mathbb{S}^{m}$ as we want; however, it is easy to transfer results from open sets to spheres (or, more generally, embedded manifolds); see the proof of Theorem 1.3 at the end of the Section 6.1.

Similarly, in the proof of Theorem 1.3 it suffices to consider the cases $p=1$ and $k \geq 3$ (by Corollary 4.3), respectively $k=2$ and $p \geq 1$. 
For further use, we note the following special case of Lemma 4.1.

4.5 Lemma. Let $1<p_{0}<p<\infty$. Then we have

$$
\|u\|_{W^{1 / p, p}} \leq C\|u\|_{W^{1 / p_{0}, p_{0}}}^{p_{0} / p}\|u\|_{L^{\infty}}^{1-p_{0} / p}, \quad \forall u \in C^{\infty}\left(\mathbb{S}^{m}\right)
$$

and

$$
|u|_{W^{1 / p, p}} \leq C|u|_{W^{1 / p_{0}, p_{0}}}^{p_{0} / p}\|u\|_{L^{\infty}}^{1-p_{0} / p}, \quad \forall u \in C^{\infty}\left(\mathbb{S}^{m}\right) .
$$

\section{Proof of Theorems 1.1 and 1.2}

In the definition of $J u$ beyond the space $W^{1, k-1}\left(\mathbb{S}^{m} ; \mathbb{S}^{k-1}\right)$, we rely on the following quantitative version of the trace theory.

5.1 Lemma. For $p>k-1$ and $u \in W^{(k-1) / p, p}\left(\mathbb{S}^{m} ; \mathbb{R}^{k}\right)$, there exists a map $v: \mathbb{S}^{m} \times[0, \infty) \rightarrow \mathbb{R}^{k}$ satisfying:

1. $v \in C^{\infty}\left(\mathbb{S}^{m} \times(0, \infty)\right)$.

2. For $T>0$, we have $v \in W^{k / p, p}\left(\mathbb{S}^{m} \times(0, T) ; \mathbb{R}^{k}\right)$ and in addition

$$
|v|_{W^{k / p, p}\left(\mathbb{S}^{m} \times(0, T)\right)} \leq C_{T}|u|_{W^{(k-1) / p, p}\left(\mathbb{S}^{m}\right)}
$$

and

$$
\int_{\mathbb{S}^{m}}|v(x, \cdot)|_{W^{k / p, p}(0, \infty)}^{p} d \mathscr{H}^{m}(x) \leq C|u|_{W^{(k-1) / p, p}\left(\mathbb{S}^{m}\right)}^{p} .
$$

3. $\operatorname{tr}_{\mid \mathbb{S}^{m} \times\{0\}} v=u$.

4. If $u \in C^{\infty}$, then $v \in C^{\infty}\left(\mathbb{S}^{m} \times[0, \infty)\right)$ and $v(\cdot, 0)=u$.

5. If $|u| \leq 1$ a.e. then $|v(x, t)| \leq 1$ and $|d v(x, t)| \leq \frac{C}{t}, \forall(x, t) \in \mathbb{S}^{m} \times(0, \infty)$.

Finally, we may choose $v$ such that the correspondence $u \mapsto v$ is linear and, in addition, $v$ does not depend on $p$.

Here,

$$
|u|_{W^{(k-1) / p, p\left(\mathbb{S}^{m}\right)}}^{p}=\int_{\mathbb{S}^{m}} \int_{\mathbb{S}^{m}} \frac{\left|u(x)-u\left(x^{\prime}\right)\right|^{p}}{d\left(x, x^{\prime}\right)^{m+k-1}} d \mathscr{H}^{m}(x) d \mathscr{H}^{m}\left(x^{\prime}\right),
$$

and the semi-norm $|v(x, \cdot)|_{W^{k / p, p}(0, \infty)}^{p}$ is given by

1. $\int_{0}^{\infty} \int_{0}^{\infty} \frac{\left|v(x, t)-v\left(x, t^{\prime}\right)\right|^{p}}{\left|t-t^{\prime}\right|^{1+k}} d t d t^{\prime}$, if $p>k$.

2. $\int_{0}^{\infty}\left|\partial_{t} v(x, t)\right|^{p} d t$, if $k=p$.

3. $\int_{0}^{\infty} \int_{0}^{\infty} \frac{\left|\partial_{t} v(x, t)-\partial_{t} v\left(x, t^{\prime}\right)\right|^{p}}{\left|t-t^{\prime}\right|^{1+k-p}} d t d t^{\prime}$, if $k-1<p<k$.

The above result is well-known to experts, but seems difficult to find in the literature. For the convenience of the reader, we present a proof of Lemma 5.1 in Section 7 .

5.2 Remark. When $p=k$, we recover the classical result that any map in $W^{1-1 / k, k}$ is the trace of a map in $W^{1, k}$. More precisely, the proof of Lemma 5.1 shows that

$$
|d v|_{L^{k}\left(\mathbb{S}^{m} \times(0, \infty)\right)} \leq C|u|_{W^{1-1 / k, k}\left(\mathbb{S}^{m}\right)} .
$$


In what follows, it will be convenient to denote by $u \mapsto T u$ the distributional Jacobian whose existence we want to establish (to be distinguished of the already existent map $u \mapsto J u$ ). Thus the conclusion of Theorem 1.11 reads $T u=J u$ if $u \in W^{1, k-1}$, and similarly Theorems 1.2 and 1.3 can be rephrased in terms of $T u$.

We now fix $p>k-1$ and $u \in W^{(k-1) / p, p}\left(\mathbb{S}^{m} ; \mathbb{S}^{k-1}\right)$ and proceed to defining $T u$. Let $\Phi \in$ $C^{\infty}\left(\mathbb{R}^{k} ; \bar{B}^{k}\right)$ be such that

$$
\Phi(X)=\frac{X}{|X|} \quad \text { if }|X| \geq \frac{1}{2} .
$$

Let $v$ be given by Lemma 5.1 and set $U:=\Phi \circ v$, which is defined in $\mathbb{S}^{m} \times[0, \infty)$, and whose trace on $\mathbb{S}^{m} \times\{0\}$ is $u$. Clearly, $U \in C^{\infty}\left(\mathbb{S}^{m} \times(0, \infty) ; \bar{B}^{k}\right)$ and $|U(x, t)|=1$ for every $(x, t) \in \mathscr{G}$, where

$$
\mathscr{G}:=\left\{(y, s) \in \mathbb{S}^{m} \times(0, \infty) ;|v(y, s)|>\frac{1}{2}\right\} .
$$

As in the proof of (1.1), we find that $U^{\sharp} \gamma=0$ on $\mathscr{G}$ for every $\gamma \in C^{\infty}\left(\Lambda^{k} \mathbb{R}^{k}\right)$; in particular, this holds for $\gamma=d y$. The key estimates in the proof of Theorems 1.1 and 1.2 are given by the following lemma, reminiscent of similar results in [10, Lemma 1, Lemma 2].

5.3 Lemma. Let $\gamma \in C_{c}^{\infty}\left(\Lambda^{k} \mathbb{R}^{k}\right)$. Then

1. $U^{\sharp} \gamma \in L^{1}\left(\mathbb{S}^{m} \times(0, \infty)\right)$ and $\left\|U^{\sharp} \gamma\right\|_{L^{1}\left(\mathbb{S}^{m} \times(0, \infty)\right)} \leq C|u|_{W^{(k-1) / p, p}\left(\mathbb{S}^{m}\right)}^{p}$.

2. $\mathscr{H}^{m}\left(\left\{x ;|v(x, t)| \leq \frac{1}{2}\right\}\right)=o\left(t^{k-1}\right)$ as $t \rightarrow 0$.

Proof. Since $\left|U^{\sharp} \gamma\right| \leq C|d v|^{k}$, we have

$$
\int_{\mathbb{S}^{m} \times(0, \infty)}\left|U^{\sharp} \gamma\right|=\int_{\mathscr{M}}\left|U^{\sharp} \gamma\right| \leq C \int_{\mathscr{M}}|d v|^{k},
$$

where $\mathscr{M}:=\left(\mathbb{S}^{m} \times(0, \infty)\right) \backslash \mathscr{G}$.

For $x \in \mathbb{S}^{m}$, we define

$$
d(x):=\inf \left\{t \in(0, \infty) ;|v(x, t)| \leq \frac{1}{2}\right\} .
$$

In particular, we have $\mathscr{M} \subset\{(x, t) ; t \geq d(x)\}$ and, if $d(x)<\infty$, then $|v(x, d(x))|=\frac{1}{2}$.

By the above and Lemma 5.15 , we find that

$$
\int_{\mathbb{S}^{m} \times(0, \infty)}\left|U^{\sharp} \gamma\right| \leq C \int_{\mathbb{S}^{m}}\left(\int_{t=d(x)}^{+\infty}|d v(x, t)|^{k} d t\right) d \mathscr{H}^{m}(x) \leq C \int_{\mathbb{S}^{m}}\left(\int_{t=d(x)}^{+\infty} \frac{d t}{t^{k}}\right) d \mathscr{H}^{m}(x),
$$

and thus

$$
\int_{\mathbb{S}^{m} \times(0, \infty)}\left|U^{\sharp} \gamma\right| \leq C \int_{\mathbb{S}^{m}} \frac{1}{d(x)^{k-1}} d \mathscr{H}^{m}(x) .
$$

For almost every $x \in \mathbb{S}^{m}$ and for every $t>0$ such that $|v(x, t)| \leq \frac{1}{2}$ we have

1. $|v(x, t)-u(x)| \geq \frac{1}{2}$.

2. $v(x, \cdot) \in W^{k / p, p}(0, \infty) \subset C^{0,(k-1) / p}(0, \infty)$.

3. $\operatorname{tr}_{\mid t=0} v(x, \cdot)=u(x)$. 
On the other hand, by the Morrey embeddings we have

$$
|v(x, t)-u(x)| \leq|v(x, \cdot)|_{C^{0,(k-1) / p}(0, t)} t^{(k-1) / p} \leq C|v(x, \cdot)|_{W^{k / p, p}(0, t)} t^{(k-1) / p} .
$$

Thus, for a.e. $x \in \mathbb{S}^{m}$ we have

$$
\frac{1}{t^{k-1}} \leq C|v(x, \cdot)|_{W^{k / p, p}(0, t)}^{p} \quad \text { if } t \geq d(x) .
$$

By taking $t=d(x)$ in (5.8) and using Lemma 5.12, this gives

$$
\int_{\mathbb{S}^{m}} \frac{1}{d(x)^{k-1}} d \mathscr{H}^{m}(x) \leq C \int_{\mathbb{S}^{m}}|v(x, \cdot)|_{W^{k / p, p}(0, \infty)}^{p} \leq C|u|_{W^{(k-1) / p, p}\left(\mathbb{S}^{m}\right)}^{p} .
$$

Lemma 5.31 is now a consequence of (5.6) and (5.9).

Finally, (5.7) implies

$$
\mathscr{H}^{m}\left(\left\{x ;|v(x, t)| \leq \frac{1}{2}\right\}\right) \leq C \int_{\mathbb{S}^{m}}|v(x, t)-u(x)|^{p} d \mathscr{H}^{m}(x) \leq C t^{k-1}|v|_{W^{k / p, p}\left(\mathbb{S}^{m} \times(0, t)\right)}^{p} .
$$

This implies Lemma 5.32 .

By Lemma 5.3, the following quantity is well-defined for every $\varsigma \in W^{1, \infty}\left(\Lambda^{m-k} \mathbb{S}^{m} \times[0, \infty)\right)$ :

$$
\langle I u, \varsigma\rangle=\frac{(-1)^{m+1}}{\omega_{k}} \int_{\mathbb{S}^{m} \times(0, \infty)}(d \varsigma) \wedge U^{\sharp} d y .
$$

The final step in defining $T u$ is provided by the following lemma.

5.4 Lemma. Let $\zeta \in W^{1, \infty}\left(\Lambda^{m-k} \mathbb{S}^{m}\right)$. Let $i$ denote the canonical inclusion map

$$
i: \mathbb{S}^{m} \rightarrow \mathbb{S}^{m} \times[0, \infty), \quad \mathbb{S}^{m} \ni x \stackrel{i}{\rightarrow}(x, 0) \in \mathbb{S}^{m} \times[0, \infty) .
$$

Let $\varsigma_{1}, \varsigma_{2} \in W^{1, \infty}\left(\Lambda^{m-k}\left(\mathbb{S}^{m} \times[0, \infty)\right)\right.$ satisfy $i^{\sharp} \varsigma_{1}=i^{\sharp} \varsigma_{2}$. Then $\left\langle I u, \varsigma_{1}\right\rangle=\left\langle I u, \varsigma_{2}\right\rangle$.

Therefore, the map

$$
\langle T u, \zeta\rangle:=\langle I u, \varsigma\rangle, \quad \forall \zeta \in W^{1, \infty}\left(\Lambda^{m-k} \mathbb{S}^{m}\right), \forall \varsigma \in W^{1, \infty}\left(\Lambda^{m-k} \mathbb{S}^{m} \times[0, \infty)\right) \text { such that } i^{\sharp} \varsigma=\zeta
$$

is well-defined.

Proof. Via a convenient partition of the unit, we may always assume that the forms we consider are supported in $\Omega$ or $\Omega \times[0, \infty)$, where $\Omega$ is a domain of local coordinates on $\mathbb{S}^{m}$. We denote the coordinates on $\Omega \times \mathbb{R}$ by $\left(y_{1}, \ldots, y_{m}, t\right)$. If $\varsigma$ is an $(m-k)$-form supported in $\Omega \times \mathbb{R}$, then we may decompose it as $\varsigma=\varsigma^{h}+\varsigma^{v}$, where $\varsigma^{h}$ represents the horizontal part (which does not contain $d t$ ), the part $\varsigma^{v}$ representing the remaining, vertical, part. The hypothesis $i^{\sharp} \varsigma_{1}=i^{\sharp} \varsigma_{2}$ is equivalent to the fact that, restricted to $\mathbb{S}^{m} \times\{0\}$, the horizontal parts $\varsigma_{1}^{h}$ and $\varsigma_{2}^{h}$ coincide. Therefore, the difference $\varsigma:=\varsigma_{1}-\varsigma_{2}$ can be approximated by a sequence $\left(\eta_{j}\right) \subset C^{\infty}\left(\Lambda^{m-k} \mathbb{S}^{m} \times[0, \infty)\right)$, supported in $\Omega \times[0, \infty)$, such that $\left(\eta_{j}\right)$ converges uniformly to $\eta,\left\|d \eta_{j}\right\|_{L^{\infty}} \leq C,\left(d \eta_{j}\right)$ converges to $d \varsigma$ a.e. on $\mathbb{S}^{m} \times(0, \infty)$ and $\eta_{j}^{h}$ vanishes in a neighborhood of $\mathbb{S}^{m} \times\{0\}$.

Fix some $j \geq 1$. Since, by the chain rule, we have $d\left(U^{\sharp} d y\right)=\left(U^{\sharp} d^{2} y\right)=0$ in $\mathbb{S}^{m} \times(0, \infty)$, and since $\eta_{j}^{h}=0$ on $\mathbb{S}^{m} \times[0, \varepsilon]$ for small $\varepsilon$, we have by Stokes' formula:

$$
\int_{\mathbb{S}^{m} \times(0, \infty)}\left(d \eta_{j}\right) \wedge\left(U^{\sharp} d y\right)=\int_{\mathbb{S}^{m} \times(\varepsilon, \infty)}\left(d \eta_{j}\right) \wedge\left(U^{\sharp} d y\right)=(-1)^{m-k+1} \int_{\mathbb{S}^{m} \times(\varepsilon, \infty)} \eta_{j} \wedge d\left(U^{\sharp} d y\right)=0 .
$$

We then let $j \rightarrow+\infty$ and find, by dominated convergence, that

$$
0=\int_{\mathbb{S}^{m} \times(0, \infty)}(d \varsigma) \wedge\left(U^{\sharp} d y\right)=\left\langle I u, \varsigma_{1}\right\rangle-\left\langle I u, \varsigma_{2}\right\rangle .
$$


Proof of Theorem 1.1 3. When $0<s<1$ and $p=\frac{k-1}{s}$, it follows from Lemma 5.31 and Lemma 5.4 that

$$
|\langle T u, \zeta\rangle| \leq C|u|_{W^{s, p}}^{p}\|d \zeta\|_{L^{\infty}}, \quad \forall \zeta \in W^{1, \infty}\left(\Lambda^{m-k} \mathbb{S}^{m}\right) .
$$

Since we will establish later that $T u=J u$ when $u \in W^{1, k-1}\left(\mathbb{S}^{m} ; \mathbb{S}^{k-1}\right)$, the above is exactly the conclusion of Theorem 1.13 when $0<s<1$. The case $s=1$ is clear from the definition of $J u$.

Proof of Theorem 1.12. We have to establish the following fact: for $k-1<p<\infty$, the map

$$
T: W^{(k-1) / p, p}\left(\mathbb{S}^{m} ; \mathbb{S}^{k-1}\right) \rightarrow\left(W^{1, \infty}\left(\Lambda^{m-k} \mathbb{S}^{m}\right)\right)^{*}, \quad u \stackrel{T}{\rightarrow} T u
$$

is continuous.

Let $\left(u_{j}\right) \subset W^{(k-1) / p, p}\left(\mathbb{S}^{m} ; \mathbb{S}^{k-1}\right)$ be a sequence converging in $W^{(k-1) / p, p}$ to some $u$. For $j \geq 1$, let $v_{j}, U_{j}=\Phi\left(v_{j}\right)$ and $d_{j}(x)$ be associated to $u_{j}$ as above, and similarly let $v, U=\Phi(v)$ and $d(x)$ be associated to $u$.

It suffices to prove that $U_{j}^{\sharp} d y$ converges to $U^{\sharp} d y$ in $L^{1}\left(\mathbb{S}^{m} \times(0, \infty)\right)$. By Lemma 5.12 , there exists $K \in L^{1}\left(\mathbb{S}^{m}\right)$ such that up, to a subsequence, we have

$$
\left|v_{j}(x, \cdot)\right|_{W^{k / p, p}(0, \infty)}^{p} \leq K(x), \quad \forall j \geq 1, \mathscr{H}^{m} \text { a.e. } x \in \mathbb{S}^{m} .
$$

As in the proof of Lemma 5.3, (5.13) leads to

$$
\frac{1}{d_{j}(x)^{k-1}} \leq C\left|v_{j}(x, \cdot)\right|_{W^{k / p, p}(0, \infty)}^{p} \leq C K(x) .
$$

Since $\left(u_{j}\right)$ converges to $u$ in $L^{p}\left(\mathbb{S}^{m}\right)$, the proof of Lemma 5.1 implies that $\left(d v_{j}\right)$ converges to $d v$ a.e., and therefore $U_{j}^{\sharp} d y$ converges to $U^{\sharp} d y$ a.e.

By (5.14), we have

$$
\left|U_{j}^{\sharp} d y\right|(x, t) \leq\left\{\begin{array}{ll}
0, & \text { if } t \leq d_{j}(x) \\
C_{0} t^{-k}, & \text { if } t>d_{j}(x)
\end{array} \leq f(x, t):= \begin{cases}0, & \text { if } t \leq(C K(x))^{-1 /(k-1)} \\
C_{0} t^{-k}, & \text { if } t>(C K(x))^{-1 /(k-1)} .\end{cases}\right.
$$

The function $f$ belongs to $L^{1}\left(\mathbb{S}^{m} \times(0, \infty)\right)$, since $K \in L^{1}\left(\mathbb{S}^{m} \times(0, \infty)\right)$. By dominated convergence, we find that $U_{j}^{\sharp} d y \rightarrow U^{\sharp} d y$ in $L^{1}\left(\mathbb{S}^{m} \times(0, \infty)\right)$, possibly up to a subsequence. Uniqueness of the limit implies that convergence occurs for the full sequence. This completes the proof of Theorem 1.12 .

Proof of Theorem 1.1 1. We start by noting that $T u$ and $J u$ make sense for some classes of not necessarily $\mathbb{S}^{k-1}$-valued maps. To start with, $J u$ is defined for $u \in W^{1, k-1} \cap L^{\infty}\left(\mathbb{S}^{m} ; \mathbb{R}^{k}\right)$, and $u \mapsto J u$ is continuous wrt $u$ in the following sense: if $u_{j} \rightarrow u$ in $W^{1, k-1}$ and $\left\|u_{j}\right\|_{L^{\infty}} \leq C$, then $J u_{j} \rightarrow J u$ in $\left(W^{1, \infty}\left(\Lambda^{m-k} \mathbb{S}^{m}\right)\right)^{*}$. On the other hand, $I u$ is defined for $u \in W^{1-1 / k, k}\left(\mathbb{S}^{m} ; \mathbb{R}^{k}\right)$ and continuous from $W^{1-1 / k, k}\left(\mathbb{S}^{m} ; \mathbb{R}^{k}\right)$ into $\left(W^{1, \infty}\left(\Lambda^{m-k} \mathbb{S}^{m} \times[0, \infty)\right)^{*}\right.$. This follows from the fact that by (5.3) we have

$$
\left|U^{\sharp} d y\right| \leq C|d U|^{k} \leq C|d v|^{k} \in L^{1}\left(\mathbb{S}^{m} \times(0, \infty)\right) .
$$

The key observation is the following.

5.5 Lemma. Tu is well-defined and the mapping $u \mapsto T u$ is continuous from $W^{1-1 / k, k}\left(\mathbb{S}^{m} ; \mathbb{R}^{k}\right)$ into $\left(W^{1, \infty}\left(\Lambda^{m-k}\left(\mathbb{S}^{m}\right)\right)^{*}\right.$.

In addition, for $u \in W^{1, k-1} \cap L^{\infty} \cap W^{1-1 / k, k}\left(\mathbb{S}^{m} ; \mathbb{R}^{k}\right)$, we have

$$
T u=J u \text {. }
$$


Proof. To start with, let $u \in C^{\infty}$. With $\omega_{0}$ the canonical volume form on $\mathbb{S}^{k-1}$ given by (1.6) and $\zeta, \varsigma$ as in Lemma 5.4, we have $d \omega_{0}=\frac{1}{\omega_{k}} d y$, and thus

$$
\begin{aligned}
\langle I u, \varsigma\rangle & =\frac{(-1)^{m+1}}{\omega_{k}} \int_{\mathbb{S}^{m} \times(0, \infty)} d \varsigma \wedge U^{\sharp} d y=(-1)^{m+1} \int_{\mathbb{S}^{m} \times(0, \infty)} d \varsigma \wedge U^{\sharp} d \omega_{0} \\
& =(-1)^{k} \int_{\mathbb{S}^{m} \times(0, \infty)} d\left(d \varsigma \wedge U^{\sharp} \omega_{0}\right)=(-1)^{m-k+1} \int_{\mathbb{S}^{m}} d \zeta \wedge u^{\sharp} \omega_{0}=\langle J u, \zeta\rangle .
\end{aligned}
$$

In the last line, we have used Stokes' formula. In particular, this proves that $\langle I u, \varsigma\rangle$ does not depend on $\varsigma$, and thus $T u$ is well-defined and $T u=J u$. By a standard approximation procedure, (5.15) remains true for a general $u$.

Finally, the continuity of $u \mapsto T u$ is clear.

Proof of Theorem 1.11 completed: the case $k \geq 3$. The Gagliardo-Nirenberg embedding

$$
W^{1, k-1} \cap L^{\infty} \subset W^{(k-1) / q, q}, \quad \forall q \geq k-1 \geq 2
$$

implies that

$$
\begin{aligned}
W^{1, k-1} \cap W^{(k-1) / p, p}\left(\mathbb{S}^{m} ; \mathbb{S}^{k-1}\right) & =W^{1, k-1}\left(\mathbb{S}^{m} ; \mathbb{S}^{k-1}\right) \\
& =W^{1, k-1} \cap W^{1-1 / k, k}\left(\mathbb{S}^{m} ; \mathbb{S}^{k-1}\right), \quad \forall p>k-1 .
\end{aligned}
$$

The preceding lemma combined with (5.16) proves that the equality $T u=J u$ holds for $u \in$ $W^{1, k-1}\left(\mathbb{S}^{m} ; \mathbb{S}^{k-1}\right)$.

Proof of Theorem 1.11 completed: the case $k=2$. This follows from Lemma 5.5 and Lemma 5.6 below.

5.6 Lemma. Let $p>1$. If $u \in W^{1,1} \cap W^{1 / p, p}\left(\mathbb{S}^{m} ; \mathbb{S}^{1}\right)$, then there exists a sequence

$$
\left(u_{j}\right) \subset W^{1,1} \cap W^{1 / 2,2} \cap W^{1 / p, p}\left(\mathbb{S}^{m} ; \mathbb{S}^{1}\right)
$$

converging to $u$ in $W^{1,1} \cap W^{1 / p, p}$.

More generally, we may pick $u_{j}$ such that $u_{j} \in W^{1, q}\left(\mathbb{S}^{m} ; \mathbb{S}^{1}\right), \forall 1<q<2$.

Proof. The result is obtained by combining the arguments in [8, Section IV] and [9, Lemma 23, Remark 5.1]. For the convenience of the reader, we sketch the proof. For $a \in \mathbb{D}$, let $\pi_{a}$ be the projection onto $\mathbb{S}^{1}$ with vertex at $a$. That is, $\pi_{a}(x)$ is the intersection with $\mathbb{S}^{1}$ of the half-line from $a$ to $x$.

Consider a regularized family $\left(u_{\varepsilon}\right)$, so that $u_{\varepsilon} \rightarrow u$ in $W^{1,1} \cap W^{1 / p, p}$ and $u_{\varepsilon} \in C^{\infty}\left(\mathbb{S}^{m} ; \overline{\mathbb{D}}\right)$. For a generic $a \in \mathbb{D}$, the set $u_{\varepsilon}^{-1}(a)$ is a smooth manifold of dimension $m-2$ and consists of regular points of $u_{\varepsilon}$. For any such $a$, the map $v_{a, \varepsilon}:=\pi_{a}\left(u_{\varepsilon}\right)$ belongs to $W^{1, q}\left(\mathbb{S}^{m} ; \mathbb{S}^{1}\right), \forall 1 \leq q<2$; see [9, Remark 5.1].

By [9, Lemma 23, Step 4], there exists a measurable set $A_{\varepsilon} \subset B(0,1 / 10) \subset \mathbb{R}^{2}$ such that $\left|A_{\varepsilon}\right|>|B(0,1 / 10)| / 2$ and for every $a_{\varepsilon} \in A_{\varepsilon}$ we have $v_{a_{\varepsilon}, \varepsilon} \rightarrow u$ in $W^{1 / p, p}\left(\mathbb{S}^{m} ; \mathbb{S}^{1}\right){ }^{23}$ On the other hand, we may pick a measurable set $B_{\varepsilon} \subset B(0,1 / 10)$ such that $\left|B_{\varepsilon}\right|>|B(0,1 / 10)| / 2$ and for every $a_{\varepsilon} \in B_{\varepsilon}$ we have $v_{a_{\varepsilon}, \varepsilon} \rightarrow u$ in $W^{1,1}\left(\mathbb{S}^{m} ; \mathbb{S}^{1}\right)\left[8\right.$, Proof of Theorem 4]. ${ }^{24}$ It then suffices to let $u_{j}=v_{a_{1 / j}, j}$ with $a_{j} \in A_{1 / j} \cap B_{1 / j}$.

${ }^{23}$ The proof there is for $p=2$, but the argument applies with no change to every $p>1$.

${ }^{24}$ The map $\pi_{a}$ in [8] is not the same as ours, but this is not relevant for the proof. 
We now turn to the proof of Theorem 1.2.

Let $u \in W^{(k-1) / p, p}\left(\mathbb{S}^{m} ; \mathbb{S}^{k-1}\right)$, and associate to $u$ the maps $v$ and $U$ as above. By the coarea formula, we have

$$
\int_{B^{k}} \mathscr{H}^{m-k+1}\left(U^{-1}(a)\right) d \mathscr{H}^{k}(a)=\int_{\mathbb{S}^{m} \times(0, \infty)}\left|U^{\sharp} d y\right| d \mathscr{H}^{m+1} .
$$

Lemma 5.3 then implies

$$
\int_{B^{k}} \mathscr{H}^{m-k+1}\left(U^{-1}(a)\right) d \mathscr{H}^{k}(a) \leq C|u|_{W^{(k-1) / p, p}\left(\mathbb{S}^{m}\right)}^{p} .
$$

Hence, for almost every $a \in B^{k}$ we have $\mathscr{H}^{m-k+1}\left(U^{-1}(a)\right)<\infty$ and we can find a large set of $a$ 's such that

$$
\mathscr{H}^{m-k+1}\left(U^{-1}(a)\right) \leq C|u|_{W^{(k-1) / p, p}}^{p} .
$$

We next establish a version of the coarea formula for differential forms.

5.7 Lemma. For a convenient orientation of $U^{-1}(y)$ and for every $\emptyset \in C^{0} \cap L^{\infty}\left(\Lambda^{m-k+1} \mathbb{S}^{m} \times\right.$ $[0, \infty))$ we have

$$
\int_{\mathbb{S}^{m} \times(0, \infty)} \varpi \wedge\left(U^{\sharp} d y\right)=\int_{B^{k}}\left(\int_{U^{-1}(y)} \varpi\right) d \mathscr{H}^{k}(y) .
$$

Proof. We first define the orientation of $U^{-1}(y)$. This is done according to the following general principle. Let $f$ be a linear map between two finite dimensional oriented vector spaces $E$ and $F$. We want to define the orientation of $\operatorname{Ker} f$. Consider a positively oriented basis $\left(\varepsilon_{1}, \ldots, \varepsilon_{r}\right)$ of $F$. Let $G$ be any supplement of $\operatorname{Ker} f$ and let $S:=f_{\mid G}: G \rightarrow F$. By convention, we say that a basis $\left(e_{1}, \ldots, e_{l}\right)$ of $\operatorname{Ker} f$ is positively oriented if $\left(e_{1}, \ldots, e_{l}, S^{-1} \varepsilon_{1}, \ldots, S^{-1} \varepsilon_{r}\right)$ is a positively oriented basis of $E$. It is easy to see that the definition of positively oriented bases of Ker $f$ does not depend on the choice of $\left(\varepsilon_{1}, \ldots, \varepsilon_{r}\right)$ or $G$. This procedure allows us to define an orientation on $U^{-1}(y)$. Indeed, if $x \in U^{-1}(y)$ is a regular point of $U$, then we have $T_{x} U^{-1}(y)=\operatorname{Ker} d U(x)$.

Let $\tau \in C^{\infty}\left(\Lambda_{m+1} \mathbb{S}^{m} \times(0, \infty)\right)$ be the oriented unit $(m+1)$-vector on $\mathbb{S}^{m} \times(0, \infty)$ and set

$$
f(x):=\left\{\begin{array}{ll}
\left\langle\omega(x) \wedge \frac{U^{\sharp} d y}{\left|U^{\sharp} d y\right|}(x), \tau(x)\right\rangle, & \text { if }\left|U^{\sharp} d y\right| \neq 0 \\
0, & \text { otherwise }
\end{array} .\right.
$$

Let $y \in \mathbb{R}^{k}$ be a regular value of $U$ and $x \in U^{-1}(y)$. Let $\left(e_{1}, \ldots, e_{m-k+1}\right)$ be a positively oriented orthonormal basis of $T_{x} U^{-1}(y)$ that we complete to a positively oriented orthonormal basis $\left(e_{1}, \ldots, e_{m+1}\right)$ of $T_{x} \mathbb{S}^{m} \times(0, \infty)$. By the convention detailed above, this means that

$$
\left(d U(x)\left(e_{m-k+2}\right), \ldots, d U(x)\left(e_{m+1}\right)\right)
$$

is a positively oriented basis of $\mathbb{R}^{k}$. In particular, $\operatorname{det}\left(\left(d U^{i}(x)\left(e_{j}\right)\right)_{i \in \llbracket 1, k \rrbracket}^{j \in \llbracket m-k+2, m+1 \rrbracket}\right)>0$.

Moreover, we have $\tau(x)=e_{1} \wedge \ldots \wedge e_{m+1}$, while $\sigma(x):=e_{1} \wedge \ldots \wedge e_{m+1-k}$ is the unit $(m+1-k)$ vector orienting $T_{x} U^{-1}(y)$. We write

$$
\varpi(x)=\sum_{1 \leq i_{1}<\ldots<i_{m+1-k} \leq m+1}{\emptyset_{i_{1}, \ldots, i_{m+1-k}}}(x) e_{i_{1}}^{*} \wedge \ldots \wedge e_{i_{m+1-k}}^{*} .
$$

We have

$$
\left(U^{\sharp} d y\right)(x)=\left(d U^{1} \wedge \ldots \wedge d U^{k}\right)(x)=\operatorname{det}\left(\left(d U^{i}(x)\left(e_{j}\right)\right)_{i \in \llbracket 1, k \rrbracket}^{j \in \llbracket m-k+2, m+1 \rrbracket}\right) e_{m-k+2}^{*} \wedge \ldots \wedge e_{m+1}^{*} .
$$

This gives

$$
\begin{aligned}
\left\langle\varrho(x) \wedge \frac{U^{\sharp} d y}{\left|U^{\sharp} d y\right|}(x), \tau(x)\right\rangle & =\varrho_{1, \ldots, m-k+1} \operatorname{sgn} \operatorname{det}\left(\left(d U^{i}(x)\left(e_{j}\right)\right)_{i \in \llbracket 1, k \rrbracket}^{j \in \llbracket m-k+2, m+1 \rrbracket}\right) \\
& =\varpi_{1, \ldots, m-k+1}=\langle\varrho(x), \sigma(x)\rangle .
\end{aligned}
$$


Therefore, we have $f(x)=\langle\varrho(x), \sigma(x)\rangle$. By the coarea formula, we find that

$$
\begin{aligned}
\int_{\mathbb{S}^{m} \times(0, \infty)} \varpi \wedge\left(U^{\sharp} d y\right) & =\int_{\mathbb{S}^{m} \times(0, \infty)} f(x) J_{U}^{\mathbb{S}^{m} \times(0, \infty)} d \mathscr{H}^{m+1}(x) \\
& =\int_{B^{k}}\left(\int_{U^{-1}(y)}\langle\Theta(x), \sigma(x)\rangle d \mathscr{H}^{m+1-k}(x)\right) d \mathscr{H}^{k}(y)=\int_{B^{k}}\left(\int_{U^{-1}(y)} \emptyset\right) d \mathscr{H}^{k}(y) .
\end{aligned}
$$

We will also need the following

5.8 Lemma. Let $\gamma \in C_{c}^{\infty}\left(\Lambda^{k-1} B^{k}\right)$. Then for every $\varsigma \in C^{1} \cap W^{1, \infty}\left(\Lambda^{m-k} \mathbb{S}^{m} \times[0, \infty)\right)$ we have

$$
\int_{\mathbb{S}^{m} \times(0, \infty)} d \varsigma \wedge U^{\sharp} d \gamma=0 .
$$

Proof. Let $t>0$. Using the fact that $\gamma$ is compactly supported in $B^{k}$, we find that $U^{\sharp} \gamma$ vanishes in the open set $\left\{x \in \mathbb{S}^{m} ;|v(x, t)|>1 / 2\right\}$, which is contained in the set $\left\{x \in \mathbb{S}^{m} ;|U(x, t)|=1\right\}$. As in the proof of (5.12), we obtain

$$
\begin{aligned}
\left|\int_{\mathbb{S}^{m} \times(t, \infty)} d \varsigma \wedge U^{\sharp} d \gamma\right| & =\left|\int_{\mathbb{S}^{m} \times\{t\}} d \varsigma \wedge U^{\sharp} \gamma\right| \\
& =\left|\int_{\left\{x \in \mathbb{S}^{m} ;|v(x, t)| \leq 1 / 2\right\} \times\{t\}} d \varsigma \wedge U^{\sharp} \gamma\right| \leq \frac{C}{t^{k-1}} \mathscr{H}^{m}\left(\left\{x \in \mathbb{S}^{m} ;|v(x, t)| \leq \frac{1}{2}\right\}\right) .
\end{aligned}
$$

This last quantity goes to 0 as $t \rightarrow 0$ by Lemma 5.32 . This implies

$$
\int_{\mathbb{S}^{m} \times(0, \infty)} d \varsigma \wedge U^{\sharp} d \gamma=\lim _{t \rightarrow 0} \int_{\mathbb{S}^{m} \times(t, \infty)} d \varsigma \wedge U^{\sharp} d \gamma=0 .
$$

We continue by presenting some consequences of Lemma 5.8. Let $\rho \in C^{\infty}\left(\bar{B}^{k}\right)$ be such that $\int_{B^{k}} \rho=\omega_{k}$ and $\rho=1$ in a neighborhood of $\mathbb{S}^{k-1}$. Then there exists $\gamma \in C_{c}^{\infty}\left(\Lambda^{k-1} B^{k}\right)$ such that $d \gamma=(1-\rho) d y$ [27, Lemma 7.5]. Hence, we can apply the above lemma to $\gamma$, which yields for every $\varsigma \in C^{1} \cap W^{1, \infty}\left(\Lambda^{m-k} \mathbb{S}^{m} \times[0, \infty)\right)$

$$
\int_{\mathbb{S}^{m} \times(0, \infty)} d \varsigma \wedge U^{\sharp} d \gamma=0 .
$$

Therefore, if we set, for every $\rho$ as above and for $\varsigma \in C^{1} \cap W^{1, \infty}\left(\Lambda^{m-k} \mathbb{S}^{m} \times[0, \infty)\right)$,

$$
I(\rho)=I(\rho, u, \varsigma):=\int_{B^{k}} \rho(y)\left(\int_{U^{-1}(y)} d \varsigma\right) d \mathscr{H}^{k}(y),
$$

then Lemma 5.7 applied to the form $(1-\rho \circ U) d \varsigma$ combined with (5.19) implies

$$
I(1)-I(\rho)=\int_{\mathbb{S}^{m} \times(0, \infty)}((1-\rho \circ U) d \varsigma) \wedge U^{\sharp} d y=\int_{\mathbb{S}^{m} \times(0, \infty)} d \varsigma \wedge U^{\sharp}((1-\rho) d y)=\int_{\mathbb{S}^{m} \times(0, \infty)} d \varsigma \wedge U^{\sharp} d \gamma=0 .
$$

We have thus proved

5.9 Lemma. For $\varsigma \in W^{1, \infty} \cap C^{1}\left(\Lambda^{m-k} \mathbb{S}^{m} \times[0, \infty)\right)$, the quantity $I(\rho)$ does not depend on the function $\rho \in C^{\infty}\left(\bar{B}^{k}\right)$ such that $\int_{B^{k}} \rho=\omega_{k}$ and $\rho=1$ in a neighborhood of $\mathbb{S}^{k-1}$. 
With $\varsigma$ fixed, let us denote by $f$ the map $y \mapsto \int_{U^{-1}(y)} d \varsigma$. By applying Lemma 5.9 with $\rho=$ $1-\sigma$, we find that, for every $\sigma \in C_{c}^{\infty}\left(B^{k}\right)$ such that $\int_{B^{k}} \sigma=0$, we have $\int_{B^{k}} \sigma(y) f(y) d \mathscr{H}^{k}(y)=0$. This implies that the function $f$ is constant almost everywhere. Hence, for every $\varsigma$, we have by Lemma 5.7 with $₫=d \varsigma$

$$
\int_{\mathbb{S}^{m} \times(0, \infty)} d \varsigma \wedge U^{\sharp} d y=\omega_{k} \int_{U^{-1}(y)} d \varsigma \quad \text { for a.e. } y \in B^{k} .
$$

By the above, (5.17) and the separability of the space $C^{1} \cap W^{1, \infty}\left(\mathbb{S}^{m} \times[0, \infty)\right)$, we obtain the following result.

5.10 Lemma. Let $p>k-1$ and $u \in W^{(k-1) / p, p}\left(\mathbb{S}^{m} ; \mathbb{S}^{k-1}\right)$. Then we can find some $a \in B^{k}$ such that

1. $U^{-1}(a)$ is a smooth $(m-k+1)$-submanifold of $\mathbb{S}^{m} \times(0, \infty)$.

2. We have

$$
\mathscr{H}^{m-k+1}\left(U^{-1}(a)\right) \leq C|u|_{W^{(k-1) / p, p}}^{p} .
$$

3. For a.e. $y \in B^{k}$ and for every $\varsigma \in C^{1} \cap W^{1, \infty}\left(\Lambda^{m-k} \mathbb{S}^{m} \times[0, \infty)\right)$ we have

$$
\int_{U^{-1}(a)} d \varsigma=\int_{U^{-1}(y)} d \varsigma
$$

Proof of Theorem 1.2. By Remark 4.4, it suffices to establish items 1 and 2 for $p>k-1$ and $u \in W^{(k-1) / p, p}\left(\mathbb{S}^{m} ; \mathbb{S}^{k-1}\right)$. We have to find an $(m-k+1)$-rectifiable current $N$ such that $T u=\partial N$. We start by defining such a current living in $\mathbb{S}^{m} \times(0, \infty)$ (this is $G$ given by (5.23)). This construction is inspired by [22]. We next project $G$ on $\mathbb{S}^{m}$; the projection given by (5.25) has all the required properties.

Consider $a$ as in Lemma 5.10. Set $\mathscr{M}:=U^{-1}(a)$, oriented as in the proof of Lemma 5.7, and let

$$
\langle G, \varpi\rangle:=(-1)^{m+1} \int_{\mathscr{M}} Ф, \quad \forall \oplus \in C^{0} \cap L^{\infty}\left(\Lambda^{m-k+1} \mathbb{S}^{m} \times[0, \infty)\right) .
$$

Then $G$ is an $(m-k+1)$-rectifiable current in $\mathbb{S}^{m} \times(0, \infty)$.

Next, let $\zeta \in C^{1}\left(\Lambda^{m-k} \mathbb{S}^{m}\right)$ and let $\varsigma \in C^{1} \cap W^{1, \infty}\left(\Lambda^{m-k} \mathbb{S}^{m} \times[0, \infty)\right)$ be a Lipschitz extension of $\zeta$. By Lemma 5.7 and Lemma 5.10 item 3, we obtain

$$
\begin{aligned}
\langle T u, \zeta\rangle & =\frac{(-1)^{m+1}}{\omega_{k}} \int_{\mathbb{S}^{m} \times(0, \infty)} d \varsigma \wedge U^{\sharp} d y=\frac{(-1)^{m+1}}{\omega_{k}} \int_{B^{k}}\left(\int_{U^{-1}(y)} d \varsigma\right) d \mathcal{H}^{k}(y) \\
& =(-1)^{m+1} \int_{U^{-1}(a)} d \varsigma=\langle G, d \varsigma\rangle=\langle\partial G, \varsigma\rangle .
\end{aligned}
$$

That is, as mentioned in the introduction, $T u$ is the boundary of a smooth $(m-k+1)$-rectifiable current living in $\mathbb{S}^{m} \times(0, \infty)$.

Next, let $\pi$ be the projection

$$
\pi: \mathbb{S}^{m} \times[0, \infty) \rightarrow \mathbb{S}^{m}, \mathbb{S}^{m} \times[0, \infty) \ni(x, t) \stackrel{\pi}{\rightarrow} x \in \mathbb{S}^{m}
$$

and set

$$
N:=\pi_{\sharp} G .
$$

The conclusion of Theorem 1.2 follows by combining Lemma 5.102 with the definition of $G$ and with the following result. 
5.11 Lemma. $N$ is an $(m-k+1)$-rectifiable current in $\mathbb{S}^{m}$. Moreover, we have

$$
\mathbf{M}(N) \leq C \mathbf{M}(G)=C \mathscr{H}^{m-k+1}(\mathscr{M})
$$

and

$$
\partial N=T u .
$$

Though the above lemma essentially follows from [21, p. 149, Section 2.4], we found useful to present a compete proof clarifying some points only sketched in [21].

Proof. As it is easy to guess, we will prove that the support of $N$ is contained in $\pi(\mathscr{M})$. In the line of Section 3, in the first part of the proof of Lemma 5.11 we establish the fact that $\pi(\mathscr{M})$ is rectifiable, and give the orientation $\xi$ and the multiplicity function $\theta$ on $\pi(\mathscr{M})$. We complete the proof of the lemma by establishing (5.27).

We start by proving that $\pi(\mathscr{M})$ is $(m-k+1)$-rectifiable. Since $\mathscr{H}^{m-k+1}(\mathscr{M})<\infty$ and $\pi$ is globally Lipschitz, we have

$$
\mathscr{H}^{m-k+1}(\pi(\mathscr{M})) \leq C \mathscr{H}^{m-k+1}(\mathscr{M})<\infty .
$$

Let $\mathscr{M}_{0}:=\left\{x \in \mathscr{M} ; J_{\pi}^{\mathscr{M}}(x)=0\right\}$. By the area formula, we have $\mathscr{H}^{m-k+1}\left(\pi\left(\mathscr{M}_{0}\right)\right)=0$. For every $x \in \mathscr{M} \backslash \mathscr{M}_{0}$, there exists an open neighborhood $U_{x} \subset \mathscr{M}$ of $x$ in $\mathscr{M}$ such that $\pi$ is a $C^{1}$ embedding from $U_{x}$ into $\mathbb{S}^{m}$. It follows that $\pi\left(U_{x}\right)$ is a $C^{1}$ manifold of dimension $m-k+1$ in $\mathbb{S}^{m}$. There exists a countable covering of $\mathscr{M} \backslash \mathscr{M}_{0}$ with such open sets $U_{x_{j}}, j \in \mathbb{N}$. This proves that $\pi(\mathscr{M})=$ $\pi\left(\mathscr{M}_{0}\right) \cup \bigcup_{j} \pi\left(U_{x_{j}}\right)$ is $(m-k+1)$-rectifiable. In order to obtain a disjoint covering, we define

$$
A_{0}:=\pi\left(U_{x_{0}}\right), A_{j}:=\pi\left(U_{x_{j}}\right) \backslash \cup_{i<j} \pi\left(U_{x_{i}}\right), \forall j \geq 1,
$$

and then we have $\pi\left(\mathscr{M} \backslash \mathscr{M}_{0}\right)=\sqcup A_{j}$.

We next define an orientation $\xi$ of $\pi(\mathscr{M})$; since $\pi\left(\mathscr{M}_{0}\right)$ is an $\mathscr{P}^{m-k+1}$-null set, it suffices to define $\xi \mathscr{H}^{m-k+1}$-a.e. in $\pi\left(\mathscr{M} \backslash \mathscr{M}_{0}\right)$. Let $\chi \in C^{\infty}\left(\Lambda_{m+1-k} \mathscr{M}\right)$ be the smooth orientation of $\mathscr{M}$ defined in the proof of Lemma 5.7. For every $x \in U_{x_{j}}, d \pi(x)$ maps $T_{x} \mathscr{M}$ onto $T_{\pi(x)} \pi\left(U_{x_{j}}\right)$. Then $\Lambda^{m-k+1} d \pi(x)(\chi(x))$ is an $(m-k+1)$-vector in $T_{\pi(x)} \pi\left(U_{x_{j}}\right)$. The unit $(m-k+1)$-vector $\frac{\Lambda^{m-k+1} d \pi(x)(\chi(x))}{\left|\Lambda^{m-k+1} d \pi(x)(\chi(x))\right|}$ gives an orientation of $\pi\left(U_{x_{j}}\right)$. However, it may happen that for some $y \in$ $\pi\left(\mathscr{M} \backslash \mathscr{M}_{0}\right)$, there exist two antecedents $z_{1}, z_{2} \in \pi^{-1}(y)$ such that the corresponding unit vectors $\frac{\Lambda^{m-k+1} d \pi\left(z_{j}\right)\left(\chi\left(z_{j}\right)\right)}{\left|\Lambda^{m-k+1} d \pi\left(z_{j}\right)\left(\chi\left(z_{j}\right)\right)\right|}, j=1,2$, do not define the same orientation. In order to define an orientation on $\pi\left(\mathscr{M} \backslash \mathscr{M}_{0}\right)$, for $y \in A_{j}$ we define the unit $(m-k+1)$-vector $\xi(y)=\frac{\Lambda^{m-k+1} d \pi(z)(\chi(z))}{\left|\Lambda^{m-k+1} d \pi(z)(\chi(z))\right|}$, where $\{z\}=\pi^{-1}(y) \cap U_{x_{j}}$. Then $\xi$ is a Borel measurable map with values into unit $(m-k+1)$ vectors on $\pi(\mathscr{M})$.

For further use, let us note that the Cauchy-Binet formula implies that

$$
\left|\left(\Lambda^{m-k+1} d \pi(x)\right) \chi(x)\right|=J_{\pi}^{\mathscr{M}}(x), \quad \forall x \in \mathscr{M}
$$

(where $|\cdot|$ denotes the Euclidean norm on the set of $(m-k+1)$-vectors, see (2.2)).

We next define the multiplicity function $\theta$. By (3.1), for $\mathscr{H}^{m-k+1}$-a.e. $y \in \pi\left(\mathscr{M} \backslash \mathscr{M}_{0}\right)$ and for every $x \in \pi^{-1}(y) \cap\left(\mathscr{M} \backslash \mathscr{M}_{0}\right)$, there exists some $\varepsilon(x) \in\{ \pm 1\}$ such that

$$
\varepsilon(x) \xi(y)=\frac{\Lambda^{m-k+1} d \pi(x)(\chi(x))}{\left|\Lambda^{m-k+1} d \pi(x)(\chi(x))\right|} .
$$

For such $y$, we let $\theta(y):=(-1)^{m+1} \sum_{x \in \pi^{-1}(y) \cap\left(\mathscr{M} \backslash \mathscr{M}_{0}\right)} \varepsilon(x)$. We start by noting that

$$
|\theta(y)| \leq \#\left[\pi^{-1}(y) \cap\left(\mathscr{M} \backslash \mathscr{M}_{0}\right)\right] .
$$


By the area formula, we have

$$
\int_{\pi(\mathscr{M})} \#\left[\pi^{-1}(y) \cap\left(\mathscr{M} \backslash \mathscr{M}_{0}\right)\right] d \mathscr{H}^{m-k+1}(y)=\int_{\mathscr{M}} J_{\pi}^{\mathcal{M}}(x) d \mathscr{H}^{m-k+1}(x) \leq C \mathscr{H}^{m-k+1}(\mathscr{M}) .
$$

This implies that $\theta$ is finite almost everywhere. We can write $\theta$ in an obvious way as a sum of countably many Borel maps taking their values in $\{-1,0,1\}$ and the above inequality also shows that this sum converges almost everywhere. Hence, $\theta$ is Borel measurable as well. In particular, the triple $(\pi(\mathscr{M}), \xi, \theta)$ defines an $(m-k+1)$-rectifiable current. In view of (5.30), the estimate (5.26) follows if we prove that this current coincides with the current $N$ given by (5.25).

$$
\begin{aligned}
& \text { If } v \in C^{\infty}\left(\Lambda^{m-k+1} \mathbb{S}^{m}\right) \text {, then } \\
& \qquad\left\langle\left(\pi^{\sharp} v\right)(x), \chi(x)\right\rangle=\left\langle v(\pi(x)), \Lambda_{m+1-k} d \pi(x) \chi(x)\right\rangle .
\end{aligned}
$$

Since by definition we have

$$
\langle N, v\rangle=\left\langle G, \pi^{\sharp} v\right\rangle, \quad \forall v \in C^{\infty}\left(\Lambda^{m-k+1} \mathbb{S}^{m}\right),
$$

we obtain, by combining (5.28), (5.29) and the area formula:

$$
\begin{aligned}
\langle N, \omega\rangle & =(-1)^{m+1} \int_{\mathscr{M} \backslash \mathscr{M}_{0}}\left\langle\omega(\pi(x)), \frac{\left(\Lambda_{m-k+1} d \pi(x)\right) \chi(x)}{\left|\left(\Lambda_{m-k+1} d \pi(x)\right) \chi(x)\right|}\right\rangle J_{\pi}^{\mathscr{M}}(x) d \mathscr{P}^{m-k+1}(x) \\
& =(-1)^{m+1} \int_{\pi(\mathscr{M})}\left\langle\omega(y), \sum_{x \in \pi^{-1}(y) \cap\left(\mathscr{M} \backslash \mathscr{M}_{0}\right)} \frac{\left(\Lambda_{m-k+1} d \pi(x)\right) \chi(x)}{\left|\left(\Lambda_{m-k+1} d \pi(x)\right) \chi(x)\right|}\right\rangle d \mathscr{H}^{m-k+1}(y) \\
& =\int_{\pi(\mathscr{M})}\langle\omega, \xi\rangle \theta d \mathscr{P}^{m-k+1} ;
\end{aligned}
$$

that is, $N$ is the current defined by the triple $(\pi(\mathscr{M}), \xi, \theta)$.

Finally, we establish (5.27). For every $\zeta \in C^{1}\left(\Lambda^{m-k} \mathbb{S}^{m}\right)$ we have

$$
\langle\partial N, \zeta\rangle=\left\langle\partial\left(\pi_{\sharp} G\right), \zeta\right\rangle=\left\langle\pi_{\sharp} G, d \zeta\right\rangle=\left\langle G, \pi^{\sharp} d \zeta\right\rangle=\left\langle G, d\left(\pi^{\sharp} \zeta\right)\right\rangle=\left\langle\partial G, \pi^{\sharp} \zeta\right\rangle=\langle T u, \zeta\rangle .
$$

Here, we rely on (5.24) and on the fact that $\varsigma=\pi^{\sharp} \zeta$ belongs to $C^{1} \cap W^{1, \infty}\left(\Lambda^{m-k} \mathbb{S}^{m} \times[0, \infty)\right)$ and satisfies (with the notations in Lemma 5.4)

$$
i^{\sharp} \varsigma=\zeta \text {. }
$$

Here is a proof of (5.31). Since $\zeta=\pi^{\sharp} \zeta$, we have $i^{\sharp} \zeta=i^{\sharp}\left(\pi^{\sharp} \zeta\right)=(\pi \circ i)^{\sharp} \zeta$. It then suffices to note that $\pi \circ i=\operatorname{id}_{\mid \mathbb{S}^{m}}$, and thus $(\pi \circ i)^{\sharp} \zeta=\zeta$. This completes the proof Lemma 5.11 and thus of Theorem 1.2.

5.12 Remark. An inspection of the proofs in this section shows that Theorems 1.1 and 1.2 still hold in bounded open subsets $\Omega$ of $\mathbb{R}^{m}$. It suffices to replace integration over $\mathbb{S}^{m}$ by integration over $\Omega$.

5.13 Remark. Let $p=k-1$ and $s=1$. For these values of $s$ and $p$, one of the results in [2] asserts that $J u=\partial N$, with $\mathbf{M}(N) \leq C_{p}|u|_{W^{s, p},}^{p}$. In addition, the proof there shows that we may build $N$ from the rectifiable set $\mathscr{N}=u^{-1}(y)$ for an appropriate $y \in \mathbb{S}^{k-1} \cdot{ }^{25}$ By the GagliardoNirenberg inequalities, the same holds when $p \in[1, k-1)$, with $s=\frac{k-1}{p}$. We do not know whether it is possible to take $N$ as above when $p>k-1$ and $s=\frac{k-1}{p}$.

\section{Proof of Theorem 1.3}

In view of Remark 4.4, it suffices to consider the cases $p=1$ and $k=2$.

${ }^{25}$ In the same way that $G$ is built from $\mathscr{M}$ in the proof of Lemma 5.11. 


\subsection{The case $p=1$}

In this case, we adapt the strategy of Alberti, Baldo and Orlandi [2]. Their approach consists in combining the dipole construction of Brezis, Coron and Lieb [13] with Federer's theorem on the approximation of integral currents with polyhedral chains [19, Theorem 4.2.20].

We first prove Theorem 1.3 when $\mathbb{S}^{m}$ is replaced by a bounded open subset $\Omega$ of $\mathbb{R}^{m}$. By Remark 5.12, $J u$ makes sense for maps defined in $\Omega$.

In Lemma 6.1 below, we describe the basic tool in the proof of Theorem 1.3: the dipole construction. This construction was first introduced in [13] and was subsequently used in [2] that we closely follow here. Since we need a dipole which is smooth (and not merely Lipschitz continuous) outside its singular set, we present, for the convenience of the reader, a detailed proof.

We say that a set $E \subset \mathbb{R}^{m}$ is an oriented $(m-k+1)$-disc when $E$ is an orientation preserving isometric linear embedding of the unit ball $B^{m-k+1} \times\left\{0_{k-1}\right\} \subset \mathbb{R}^{m}$, endowed with the canonical orientation. We will often write $|E|=\mathscr{P}^{m-k+1}(E)$.

6.1 Lemma. Let $E \subset \mathbb{R}^{m}$ be an oriented $(m-k+1)$-disc with center $x$ and radius $r$. Let $\sigma>0$. Then there exists $u \in W_{l o c}^{k-1,1}\left(\mathbb{R}^{m} ; \mathbb{S}^{k-1}\right) \cap C^{\infty}\left(\mathbb{R}^{m} \backslash \partial E\right)$ such that

1. $J u=\partial E$.

2. There exists $Y \in \mathbb{S}^{k-1}$ such that $u=Y$ in $\mathbb{R}^{m} \backslash \bar{B}(x, r)$ and

$$
\left|\left\{y \in \mathbb{R}^{m}: u(y) \neq Y\right\}\right| \leq \sigma .
$$

3. There exists $C_{\sigma}>0$ such that

$$
\left|D^{l} u(y)\right| \leq \frac{C_{\sigma}}{\operatorname{dist}(y, \partial E)^{l}}, \quad \forall y \in \mathbb{R}^{m} \backslash \partial E, \forall l \in \mathbb{N} .
$$

4. There exists $C>0$ such that

$$
\left\|D^{l} u\right\|_{L^{1}\left(\mathbb{R}^{m}\right)} \leq C|E|^{(m-l) /(m-k+1)}, \quad \forall l \in \llbracket 1, k-1 \rrbracket .
$$

Here, $\partial E$ is the boundary of $E$ considered as a manifold with boundary.

Proof. We start with a remark in the spirit of [2, Section 7.2]: if $\varphi: \mathbb{R}^{m} \rightarrow \mathbb{R}^{m}$ is a smooth diffeomorphism, and if $u \in W^{1, k-1} \cap L^{\infty}$, then $\varphi^{\sharp} J u=J\left(\varphi^{\sharp} u\right)$. Indeed, for every $\zeta \in C_{c}^{\infty}\left(\Lambda^{m-k} \mathbb{R}^{m}\right)$ we have

$$
\int_{\mathbb{R}^{m}} d\left(\varphi_{\sharp} \zeta\right) \wedge u^{\sharp} \omega_{0}=\int_{\mathbb{R}^{m}} \varphi_{\sharp}\left(d \zeta \wedge \varphi^{\sharp}\left(u^{\sharp} \omega_{0}\right)\right)=\int_{\mathbb{R}^{m}} d \zeta \wedge\left(\left(\varphi^{\sharp} u\right)^{\sharp} \omega_{0}\right),
$$

which is equivalent to $\left\langle\varphi^{\sharp} J u, \zeta\right\rangle=\left\langle J\left(\varphi^{\sharp} u\right), \zeta\right\rangle$ and implies that $\varphi^{\sharp} J u=J\left(\varphi^{\sharp} u\right)$.

By taking $\varphi$ an affine homothety, we see that in the proof of Lemma 6.1 we may assume that $E=B^{m-k+1} \times\left\{0_{k-1}\right\} \subset \mathbb{R}^{m}$.

Let $P: B^{k-1} \rightarrow \mathbb{S}^{k-1}$ be a smooth map of degree $(-1)^{m-k+1}$ such that $P$ is constant near $\mathbb{S}^{k-2}=\partial B^{k-1}$. For instance, with || standing for the Euclidean norm and $\mu \in(0,1 / 10)$ a fixed parameter, a suitable $P$ is given by

$$
P(x):=\left(f(\sin \pi|x|) \frac{x}{|x|},(-1)^{m} h(|x|) \cos (\pi|x|)\right),
$$

where $f: \mathbb{R}^{+} \rightarrow \mathbb{R}^{+}$is a smooth nondecreasing function which vanishes on $(0, \mu)$ and is equal to the identity on $(2 \mu, \infty)$ and for $r \in[0,1]$

$$
h(r):=\left\{\begin{array}{ll}
\left(\frac{1-(f(\sin \pi r))^{2}}{\cos ^{2} \pi r}\right)^{1 / 2}, & \text { if } r \neq \frac{1}{2} \\
1, & \text { if } r=\frac{1}{2}
\end{array} .\right.
$$


Note that when $|x|$ is close to 0 , the first coordinate of $P$ is 0 , while the second one is equal to $(-1)^{m}$. Hence $P$ is smooth despite the singularity of $x \mapsto|x|$ at the origin.

We claim that $\operatorname{deg} P=(-1)^{m-k+1}$. Indeed, let $x=(t, 0, \ldots, 0) \in B^{k-1}$ be such that $\sin \pi t>2 \mu$. Then $P^{-1}(P(x))=\{x\}$, and thus $\operatorname{deg} P$ is given by the orientation of $d_{x} P$. Since the orientation of $\mathbb{S}^{k-1}$ is given by $\omega_{0}$ and the one of $\mathbb{R}^{k-1}$ by $d x_{1} \wedge \ldots \wedge d x_{k-1}$, and in addition we have (with $x$ as above)

$$
P^{\sharp} \omega_{0}(x)=\frac{(-1)^{m-k+1}(\sin \pi t)^{k-2}}{k \omega_{k} t^{k-2}} \pi d x_{1} \wedge \ldots \wedge d x_{k-1},
$$

we find that $\operatorname{deg} P=(-1)^{m-k+1}$, as claimed.

When $|x|$ is close to 1 , the first coordinate of $P$ vanishes and the second one is equal to $(-1)^{m+1}$. Thus, $P$ is constant on a neighborhood of $\mathbb{S}^{k-2}$, where it takes the value $Y:=$ $\left(0_{k-1},(-1)^{m+1}\right)$. Let $\eta>0$ be such that $P(x)=Y$ when $|x|>1-\eta$.

Let $\delta \in(0,1 / 2)$ to be specified later and let $q:[0,1] \rightarrow[0, \delta]$ be a smooth nonincreasing function such that $q(t)=\delta$ on $[0,1-2 \delta], q(t)=1-t$ on $[1-\delta / 2,1], q(t) \leq 1-t$ on $[0,1]$. Moreover, we require that the derivatives of $q^{(l)}$ can be bounded independently of $\delta$.

For every $x \in \mathbb{R}^{m}$, we write $x=\left(x^{\prime}, x^{\prime \prime}\right) \in \mathbb{R}^{m-k+1} \times \mathbb{R}^{k-1}$. We define

$$
u(x):= \begin{cases}P\left(\frac{x^{\prime \prime}}{q\left(\left|x^{\prime}\right|\right)}\right), & \text { if }\left|x^{\prime}\right|<1 \text { and }\left|x^{\prime \prime}\right|<q\left(\left|x^{\prime}\right|\right) \\ Y, & \text { otherwise }\end{cases}
$$

The map $u$ is smooth in

$$
\mathbb{R}^{m} \backslash \partial E=\mathbb{R}^{m} \backslash\left(\mathbb{S}^{m-k} \times\left\{0_{k-1}\right\}\right)
$$

and we have $u=Y$ in the open set

$$
V:=\left\{\left(x^{\prime}, x^{\prime \prime}\right) ;\left|x^{\prime}\right|<1,\left|x^{\prime \prime}\right|>(1-\eta) q\left(\left|x^{\prime}\right|\right)\right\} \cup\left\{\left(x^{\prime}, x^{\prime \prime}\right) ;\left|x^{\prime}\right|>1\right\},
$$

which is a neighborhood of $\mathbb{S}^{m-1} \backslash\left(\mathbb{S}^{m-k} \times\left\{0_{k-1}\right\}\right)$. Moreover, we have

$$
|\{x ; u(x) \neq Y\}| \leq\left|\left\{x=\left(x^{\prime}, x^{\prime \prime}\right) ;\left|x^{\prime \prime}\right|<q\left(\left|x^{\prime}\right|\right),\left|x^{\prime}\right|<1\right\}\right| \leq C_{0} \delta^{k-1} .
$$

We choose $\delta>0$ such that $C_{0} \delta^{k-1}<\sigma$. This implies (6.1).

Next, for every $l \geq 0$ there exists $C_{l}>0$ independent of $\delta$ such that

$$
\left|D^{l} u(x)\right| \leq \frac{C_{l}}{q\left(\left|x^{\prime}\right|\right)^{l}}, \quad \forall x \notin \mathbb{S}^{m-k} \times\left\{0_{k-1}\right\} .
$$

In view of the definition of $q$, this implies the item 3 and also the fact that $u \in W_{l o c}^{k-1,1}\left(\mathbb{R}^{m} ; \mathbb{S}^{k-1}\right)$. As a consequence of (6.5), we have for every $l \in \llbracket 1, k-1 \rrbracket$

$$
\int_{\mathbb{R}^{m}}\left|D^{l} u(x)\right| d x \leq C_{l} \int_{B^{m-k+1}}\left(\int_{\left|x^{\prime \prime}\right|<q\left(\left|x^{\prime}\right|\right)} d x^{\prime \prime}\right) \frac{d x^{\prime}}{q\left(\left|x^{\prime}\right|\right)^{l}} \leq C_{l}^{\prime} \delta^{k-l-1},
$$

which implies item 4.

It remains to prove item 1 which follows from the following explicit calculation. ${ }^{26}$ We introduce, for $\varepsilon \in(0, \delta / 2)$, the open set

$$
T(\varepsilon):=\left\{\left(x^{\prime}, x^{\prime \prime}\right) \in \mathbb{R}^{m-k+1} \times \mathbb{R}^{k-1} ;|1-| x^{\prime}||<\varepsilon,\left|x^{\prime \prime}\right|<2 \varepsilon\right\} .
$$

Let $\zeta \in C_{c}^{\infty}\left(\Lambda^{m-k} \mathbb{R}^{m}\right)$. By dominated convergence, we have

$$
\langle J u, \zeta\rangle=(-1)^{m-k+1} \lim _{\varepsilon \rightarrow 0} \int_{\mathbb{R}^{m} \backslash T(\varepsilon)} d \zeta \wedge u^{\sharp} \omega_{0} .
$$

\footnotetext{
${ }^{26}$ Alternatively, we could have invoked Jerrard and Soner [25, Theorem 1].
} 
In $\mathbb{R}^{m} \backslash T(\varepsilon), u$ is smooth and $\mathbb{S}^{k-1}$-valued, so that $d\left(u^{\sharp} \omega_{0}\right)=0$. Stokes' formula then implies

$$
\langle J u, \zeta\rangle=(-1)^{m-k} \lim _{\varepsilon \rightarrow 0} \int_{\partial T(\varepsilon)} \zeta \wedge u^{\sharp} \omega_{0} .
$$

Since $u$ is constant in the open set $V$ given by (6.4), we have

$$
\int_{\partial T(\varepsilon)} \zeta \wedge u^{\sharp} \omega_{0}=\int_{\partial T(\varepsilon) \backslash V} \zeta \wedge u^{\sharp} \omega_{0} .
$$

Let, in the remaining part of the proof, $\bar{B}^{l}(x, r)$ denote the closed ball $\bar{B}(x, r) \subset \mathbb{R}^{l}$ and set $\bar{B}_{r}^{l}=$ $\bar{B}^{l}(0, r)$; we use similar notation for the open balls. Observe that

$$
\partial T(\varepsilon) \backslash V \subset\left\{\left((1-\varepsilon) x^{\prime}, x^{\prime \prime}\right) ; x^{\prime} \in \mathbb{S}^{m-k}, x^{\prime \prime} \in \bar{B}_{2 \varepsilon}^{k-1}\right\}
$$

and that, on $\partial T(\varepsilon) \backslash V, u$ depends only on $x^{\prime \prime}$. Using (6.5) and the properties of $q$, we find that

$$
\left\|u^{\sharp} \omega_{0}\right\|_{L^{\infty}(\partial T(\varepsilon) \backslash V)} \leq C / \varepsilon^{k-1} .
$$

Recall that, if $M \subset U$ (with $M$ manifold and $U$ open set), then we denote by $\zeta_{\mid M}$ the pullback $i \sharp \zeta$ of a form on $U$; here, $i: M \rightarrow U$ is the inclusion. With this notation and using the fact that, on $\partial T(\varepsilon) \backslash V, u$ depends only on $x^{\prime \prime}$, for every $\left((1-\varepsilon) x^{\prime}, x^{\prime \prime}\right)$ as in (6.9) we have

$$
\left.\left(\zeta \wedge u^{\sharp} \omega_{0}\right)\right|_{\partial T(\varepsilon) \backslash V}\left((1-\varepsilon) x^{\prime}, x^{\prime \prime}\right)=\zeta_{\mid(1-\varepsilon) \mathbb{S}^{m-k} \times\left\{x^{\prime \prime}\right\}} \wedge\left(u^{\sharp} \omega_{0}\right)_{\mid\left\{(1-\varepsilon) x^{\prime}\right\} \times \bar{B}_{2 \varepsilon}^{k-1}}\left((1-\varepsilon) x^{\prime}, x^{\prime \prime}\right) .
$$

Assume for a moment that $\zeta_{\mathbb{S}^{m-k} \times\{0\}}=0$. By combining the estimate $\mathscr{H}^{m-k}(\partial T(\varepsilon) \backslash V) \leq C \varepsilon^{k-1}$ with (6.10), we find that

$$
\left|\int_{\partial T(\varepsilon) \backslash V} \zeta \wedge u^{\sharp} \omega_{0}\right| \leq C \max _{\substack{\left|x^{\prime}\right|=1 \\\left|x^{\prime \prime}\right| \leq 2 \varepsilon}}\left|\zeta_{\mid(1-\varepsilon) \mathbb{S}^{m-k} \times\left\{x^{\prime \prime}\right\}}\left(x^{\prime}, x^{\prime \prime}\right)\right|,
$$

which implies

$$
\lim _{\varepsilon \rightarrow 0} \int_{\partial T(\varepsilon) \backslash V} \zeta \wedge u^{\sharp} \omega_{0}=0 .
$$

We now turn to a general $\zeta \in C_{c}^{\infty}\left(\Lambda^{m-k} \mathbb{R}^{m}\right)$. If $p$ is the projection

$$
p:\left(\mathbb{R}^{m-k+1} \backslash\{0\}\right) \times \mathbb{R}^{k-1} \rightarrow \mathbb{S}^{m-k},\left(\mathbb{R}^{m-k+1} \backslash\{0\}\right) \times \mathbb{R}^{k-1} \ni x=\left(x^{\prime}, x^{\prime \prime}\right) \stackrel{p}{\rightarrow} \frac{x^{\prime}}{\left|x^{\prime}\right|} \in \mathbb{S}^{m-k},
$$

then we have

$$
\left(\zeta-p^{\sharp}\left(\zeta_{\mid \mathbb{S}^{m-k} \times\left\{0_{k-1}\right\}}\right)\right)_{\mid \mathbb{S}^{m-k} \times\left\{0_{k-1}\right\}}=0 .
$$

In view of (6.11) and (6.12), we have

$$
\lim _{\varepsilon \rightarrow 0}\left(\int_{\partial T(\varepsilon) \backslash V} \zeta \wedge u^{\sharp} \omega_{0}-\int_{\partial T(\varepsilon) \backslash V} p^{\sharp}\left(\zeta_{\mathbb{S}^{m-k} \times\left\{0_{k-1}\right\}}\right) \wedge u^{\sharp} \omega_{0}\right)=0 .
$$

From the above, we may thus assume that $\zeta$ is of the form $p^{\sharp} \widetilde{\zeta}$ with $\widetilde{\zeta} \in C^{\infty}\left(\Lambda^{m-k} \mathbb{S}^{m-k}\right)$, which we henceforth do.

Let us introduce the diffeomorphism $\psi:\left[(1-\varepsilon) \mathbb{S}^{m-k}\right] \times \mathbb{R}^{k-1} \rightarrow \mathbb{S}^{m-k} \times \mathbb{R}^{k-1}$,

$$
\left[(1-\varepsilon) \mathbb{S}^{m-k}\right] \times \mathbb{R}^{k-1} \ni x=\left(x^{\prime}, x^{\prime \prime}\right) \stackrel{\psi}{\rightarrow}\left(\frac{x^{\prime}}{\left|x^{\prime}\right|}, x^{\prime \prime}\right) \in \mathbb{S}^{m-k} \times \mathbb{R}^{k-1},
$$

and the projection

$$
\pi: \mathbb{S}^{m-k} \times \mathbb{R}^{k-1} \rightarrow \mathbb{R}^{k-1}, \mathbb{S}^{m-k} \times \mathbb{R}^{k-1} \ni x=\left(x^{\prime}, x^{\prime \prime}\right) \stackrel{\pi}{\rightarrow} x^{\prime \prime} \in \mathbb{R}^{k-1} .
$$


Observe that the orientation of $(1-\varepsilon) \mathbb{S}^{m-k} \times\left\{0_{k-1}\right\}$ is defined by the orientation of $\partial T(\varepsilon)$, while $\mathbb{S}^{m-k}$ is endowed with its standard orientation. Hence, the maps $p$ and $\pi$ preserve the orientation whereas $\psi$ does not.

Let $v\left(x^{\prime \prime}\right)=P\left(\frac{x^{\prime \prime}}{\varepsilon}\right)$. Observe that

$$
\int_{\mathbb{R}^{k-1}} v^{\sharp} \omega_{0}=(-1)^{m-k+1} .
$$

Moreover, we have $\psi_{\sharp} u=\pi^{\sharp} v$ and $\psi_{\sharp} \zeta=\left(p^{\sharp} \widetilde{\zeta}\right)_{\mid \mathbb{S}^{m-k} \times \mathbb{R}^{k-1}}$.

By (6.13), the fact that $u^{\sharp} \omega_{0}=0$ in $V$ and Fubini's theorem for differential forms [27, Lemma 6.32] we have

$$
\begin{aligned}
\int_{\partial T(\varepsilon) \backslash V} \zeta \wedge u^{\sharp} \omega_{0} & =\int_{\left[(1-\varepsilon) \mathbb{S}^{m-k}\right] \times \mathbb{R}^{k-1}} \zeta \wedge u^{\sharp} \omega_{0}=-\int_{\mathbb{S}^{m-k} \times \mathbb{R}^{k-1}} \psi_{\sharp}\left(\zeta \wedge\left(u^{\sharp} \omega_{0}\right)\right) \\
& =-\int_{\mathbb{S}^{m-k} \times \mathbb{R}^{k-1}} p^{\sharp} \tilde{\zeta} \wedge \pi^{\sharp}\left(v^{\sharp} \omega_{0}\right)=-\int_{\mathbb{S}^{m-k}}\left(\int_{\mathbb{R}^{k-1}} v^{\sharp} \omega_{0}\right) \tilde{\zeta}=(-1)^{m-k} \int_{\mathbb{S}^{m-k} \times\left\{0_{k-1}\right\}} \zeta .
\end{aligned}
$$

In view of (6.7) and (6.8), this completes the proof of Lemma 6.1.

In order to proceed with the proof of Theorem 1.3, let us recall the following quantitative version of Federer's approximation theorem, devised by Alberti, Baldo and Orlandi [2, Corollary 7.13].

6.2 Lemma. Let $R$ be an $(m-k+1)$-rectifiable current in an open set $\Omega \subset \mathbb{R}^{m}$, and $\rho$ a function in $\Omega$ which is positive $\mathscr{P}^{m-k+1}$-a.e. Then there exist finitely many oriented $(m-k+1)$-discs $E_{j}$ with centers $x_{j}$ and radii $r_{j}<\rho\left(x_{j}\right)$ and an $(m-k+1)$-rectifiable current $T$ such that

1. $\partial R=\sum \partial E_{j}+\partial T$.

2. $\sum\left|E_{j}\right| \leq \frac{1}{2^{m-k}} \mathbf{M}(R)$.

3. $\mathbf{M}(T) \leq\left(1-\frac{1}{2^{m-k+2}}\right) \mathbf{M}(R)$.

4. The balls $\bar{B}^{m}\left(x_{j}, 2 r_{j}\right)$ are pairwise disjoint and contained in $\Omega$.

6.3 Corollary. Let $N$ be an $(m-k+1)$-rectifiable current in $\Omega \subset \mathbb{R}^{m}$. Then there exists a sequence of finite families of oriented $(m-k+1)$-discs $\left(E_{j, l}\right)_{j \in \llbracket 1, N(l) \rrbracket}$, of centers $x_{j, l}$ and radii $r_{j, l}$, such that:

1. $\partial N=\sum_{l} \sum_{j} \partial E_{j, l}$.

2. $\sum_{j}\left|E_{j, l}\right| \leq \frac{1}{2^{m-k}}\left(1-\frac{1}{2^{m-k+2}}\right)^{l-1} \mathbf{M}(N), \forall l \geq 1$. In particular, $\sum_{l} \sum_{j}\left|E_{j, l}\right| \leq 4 \mathbf{M}(N)$.

3. For a fixed $l$, the balls $\bar{B}^{m}\left(x_{j, l}, 2 r_{j, l}\right)$ are pairwise disjoint and contained in $\Omega$.

Proof. We first apply Lemma 6.2 with $R=N$. This yields a family $\left(E_{j, 1}\right)$ and a reminder $T_{1}$. We next apply the lemma with $R=T_{1}$ and obtain a family $\left(E_{j, 2}\right)$, and so on. It is straightforward that the families obtained by this procedure have all the required properties.

We obtain our next result by combining Lemma 6.1 and Lemma 6.2 and by adapting the proof of [2, Theorem 5.6].

6.4 Lemma. Let $R$ be an $(m-k+1)$-rectifiable current in a bounded open set $\Omega \subset \mathbb{R}^{m}$. Let $u \in W^{k-1,1}\left(\Omega ; \mathbb{S}^{k-1}\right)$ be smooth in the complement of a closed set $S \subset \Omega$ such that $\mathscr{P}^{m-k}(S)<\infty$. Then there exist an $(m-k+1)$-rectifiable current $T$ in $\Omega$ and a map $v \in W^{k-1,1}\left(\Omega ; \mathbb{S}^{k-1}\right)$, smooth in the complement of a closed set $S^{\prime} \subset \Omega$ such that $\mathscr{H}^{m-k}\left(S^{\prime}\right)<\infty$, satisfying:

1. $J v=J u+\partial R-\partial T$. 

2. $\left\|D^{l} u-D^{l} v\right\|_{L^{1}(\Omega)} \leq C \mathbf{M}(R), \forall l \in \llbracket 0, k-1 \rrbracket$.
3. $\mathbf{M}(T) \leq\left(1-\frac{1}{2^{m-k+2}}\right) \mathbf{M}(R)$.

Proof. For the convenience of the reader, we explain how the argument in [2, Proof of Theorem $5.6]$ adapts to our case. We divide the proof into several steps.

Step 1. Choice of $\rho$.

Since $S$ is closed, for every $x \in \Omega \backslash S$ we can find $r=r(x)>0$ such that $\bar{B}^{m}(x, r) \subset \Omega \backslash S$. Since $u$ is smooth on the complement of $S, D^{l} u$ is bounded in $B^{m}(x, r)$ for every integer $l$. We let $\rho: \Omega \rightarrow[0,+\infty)$ be defined by $\rho(x)=0$ for $x \in S$ and by

$$
0<\rho(x) \leq \min \left\{\frac{r}{2},\left(\frac{1}{\omega_{m-k+1}}\right)^{1 /(m-k+1)}, \min _{1 \leq l \leq k-1} \frac{1}{8\left\|D^{l} u\right\|_{L^{\infty}\left(B^{m}(x, r)\right)}^{1 / l}}\right\}, \quad \forall x \in \Omega \backslash S .
$$

We next apply Lemma 6.2 with the above $\rho$ and obtain finitely many discs $E_{j}$, with centers $x_{j} \in \Omega \backslash S$ and radii $r_{j}<\rho\left(x_{j}\right)$, and an $(m-k+1)$-rectifiable current $T$ in $\Omega$ such that:

$$
\begin{aligned}
& \partial R=\sum \partial E_{j}+\partial T, \\
& \sum_{j}\left|E_{j}\right| \leq \frac{1}{2^{m-k}} \mathbf{M}(R), \\
& \mathbf{M}(T) \leq\left(1-\frac{1}{2^{m-k+2}}\right) \mathbf{M}(R),
\end{aligned}
$$

and the balls $\bar{B}^{m}\left(x_{j}, 2 r_{j}\right)$ are pairwise disjoint and contained in $\Omega$.

Step 2. Construction of $v$.

For every $j$, we construct a dipole $u_{j}$ associated to $E_{j}$ as in Lemma 6.1 with the parameter $\sigma=1$. Thus $J u_{j}=\partial E_{j}$, there exists $Y_{j} \in \mathbb{S}^{k-1}$ such that $u_{j}=Y_{j}$ in $\mathbb{R}^{m} \backslash B^{m}\left(x_{j}, r_{j}\right)$, and

$$
\left|D^{l} u_{j}(x)\right| \leq \frac{C_{l}}{\operatorname{dist}\left(x, \partial E_{j}\right)^{l}}, \quad \forall x \in \mathbb{R}^{m} \backslash \partial E_{j}, \forall l \in \mathbb{N} .
$$

Note that the choice of $Y_{j}$ is not relevant in the above construction. Therefore, we may assume that $Y_{j}=u\left(y_{j}\right)$ for some $y_{j} \in B^{m}\left(x_{j}, 2 r_{j}\right)$. Let $\theta \in C_{c}^{\infty}\left(B_{2}^{m}\right)$ be such that $0 \leq \theta \leq 1$ and $\theta \equiv 1$ in $\bar{B}_{1}^{m}$. Then we define $\theta_{j}(x):=\theta\left(\frac{x-x_{j}}{r_{j}}\right)$ and

$$
W:=\left\{\begin{array}{ll}
\theta_{j} u_{j}+\left(1-\theta_{j}\right) u & \text { in } B^{m}\left(x_{j}, 2 r_{j}\right) \\
u & \text { in } \Omega \backslash \cup_{j} B^{m}\left(x_{j}, 2 r_{j}\right)
\end{array} .\right.
$$

We have

$$
\left\|u_{j}-u\right\|_{L^{\infty}\left(B^{m}\left(x_{j}, 2 r_{j}\right) \backslash B^{m}\left(x_{j}, r_{j}\right)\right)} \leq 4 r_{j}\|D u\|_{L^{\infty}\left(B^{m}\left(x_{j}, 2 r_{j}\right)\right)}<\frac{1}{2} .
$$

Here, we have used the fact that $u_{j} \equiv Y_{j}$ in $B^{m}\left(x_{j}, 2 r_{j}\right) \backslash B^{m}\left(x_{j}, r_{j}\right)$ and that $u\left(y_{j}\right)=Y_{j}$ for some $y_{j} \in B^{m}\left(x_{j}, 2 r_{j}\right)$, and also the inequality

$$
r_{j}<\frac{1}{8\|D u\|_{L^{\infty}\left(B^{m}\left(x_{j}, 2 r_{j}\right)\right)}} .
$$

In view of (6.19) and of the properties of $\theta$, we have $|W| \geq 1 / 2$ and thus we may define the $\mathbb{S}^{k-1}$-valued map $v:=\frac{W}{|W|}$. 
Step 3. The map $v$ has all the required properties.

On the one hand, $v$ agrees with $u_{j}$ in $B^{m}\left(x_{j}, r_{j}\right)$; on the other hand, we have $u \in W^{k-1,1}\left(\Omega ; \mathbb{S}^{k-1}\right)$. Therefore, the map $v$ belongs to $W^{k-1,1}\left(\Omega ; \mathbb{S}^{k-1}\right)$. Next, $v$ is smooth outside $S^{\prime}:=S \cup \cup_{j} \partial E_{j}$. Moreover, since the Jacobian of $u$ is supported in $S$, and since $S \cap \cup_{j} \partial E_{j}=\varnothing$, we have

$$
J v=J u+\sum_{j} J u_{j}=J u+\sum \partial E_{j} .
$$

The first equality in (6.20) is easily obtained using (1.7) and a partition of the unit. We obtain items 1 and 3 by combining (6.15), (6.17) and (6.20).

It remains to prove item 2 . For $l \in \llbracket 0, k-1 \rrbracket$ we have

$$
\left\|D^{l} u-D^{l} v\right\|_{L^{1}(\Omega)}=\sum_{j}\left\|D^{l} u-D^{l} v\right\|_{L^{1}\left(B^{m}\left(x_{j}, 2 r_{j}\right)\right)} \leq \sum_{j}\left(\left\|D^{l} u\right\|_{L^{1}\left(B^{m}\left(x_{j}, 2 r_{j}\right)\right)}+\left\|D^{l} v\right\|_{L^{1}\left(B^{m}\left(x_{j}, 2 r_{j}\right)\right)}\right) .
$$

When $l=0$, we have $|v(x)|=|u(x)|=1$ a.e., and therefore

$$
\|u-v\|_{L^{1}(\Omega)} \leq C \sum_{j} r_{j}^{m} \leq C \sum_{j}\left|E_{j}\right|^{m /(m-k+1)} \leq C \sum\left|E_{j}\right| \leq C \mathbf{M}(R) .
$$

Here, we have used (6.16) and the fact that (by (6.14)) we have $\left|E_{j}\right| \leq 1$.

We now assume that $1 \leq l \leq k-1$. On the one hand, we have

$$
\left\|D^{l} u\right\|_{L^{1}\left(B^{m}\left(x_{j}, 2 r_{j}\right)\right)} \leq C r_{j}^{m}\left\|D^{l} u\right\|_{L^{\infty}\left(B^{m}\left(x_{j}, 2 r_{j}\right)\right)} \leq C r_{j}^{m-l} \leq C\left|E_{j}\right|^{(m-l) /(m-k+1)},
$$

by definition of $\rho$. As above, we find that $\left\|D^{l} u\right\|_{L^{1}\left(B^{m}\left(x_{j}, 2 r_{j}\right)\right)} \leq C\left|E_{j}\right|$, and thus

$$
\sum_{j}\left\|D^{l} u\right\|_{L^{1}\left(B^{m}\left(x_{j}, 2 r_{j}\right)\right)} \leq C \mathbf{M}(R) .
$$

On the other hand, by the Faà-Di Bruno formula,

$$
\left|D^{l} v(x)\right| \leq C \sum_{s=1}^{l} \sum_{t_{1}+\ldots+t_{s}=l}\left|D^{t_{1}} W(x)\right| \ldots\left|D^{t_{s}} W(x)\right| .
$$

For every $t \in \llbracket 1, l \rrbracket$ and $x \in B^{m}\left(x_{j}, 2 r_{j}\right)$, the Leibniz formula implies

$$
\left|D^{t} W(x)\right| \leq\left|D^{t} u(x)\right|+\left|D^{t}\left[\theta_{j}\left(u_{j}-u\right)\right](x)\right| \leq\left|D^{t} u(x)\right|+C \sum_{h=0}^{t}\left|D^{t-h} \theta_{j}(x)\right|\left[\left|D^{h} u_{j}(x)\right|+\left|D^{h} u(x)\right|\right] .
$$

In view of the definitions of $\theta_{j}$ and $\rho$ we obtain, via (6.18):

$$
\left|D^{t} W(x)\right| \leq C \sum_{h=0}^{t} \frac{1}{r_{j}^{t-h}}\left[\frac{1}{r_{j}^{h}}+\left|D^{h} u_{j}(x)\right|\right] \leq C \sum_{h=0}^{t} \frac{1}{r_{j}^{t-h}} \frac{1}{\operatorname{dist}\left(x, \partial E_{j}\right)^{h}} .
$$

Inserting this inequality into (6.23), we obtain

$$
\left|D^{l} v(x)\right| \leq C \sum_{s=1}^{l} \sum_{t_{1}+\ldots+t_{s}=l} \sum_{\substack{0 \leq h_{p} \leq t_{p} \\ \forall p \in \llbracket 1, s \rrbracket}} \frac{1}{r_{j}^{l-h_{1}-\ldots-h_{s}}} \frac{1}{\operatorname{dist}\left(x, \partial E_{j}\right)^{h_{1}+\ldots+h_{s}}}, \quad \forall x \in B^{m}\left(x_{j}, 2 r_{j}\right) .
$$

By scaling, we easily find that

$$
\int_{B^{m}\left(x_{j}, 2 r_{j}\right)} \frac{d x}{\operatorname{dist}\left(x, \partial E_{j}\right)^{h_{1}+\ldots+h_{s}}} \leq C r_{j}^{m-h_{1}-\ldots-h_{s}},
$$

and thus

$$
\int_{B^{m}\left(x_{j}, 2 r_{j}\right)}\left|D^{l} v(x)\right| d x \leq C r_{j}^{m-l} \leq C\left|E_{j}\right|^{(m-l) /(m-k+1)} \leq C\left|E_{j}\right| .
$$

Finally, with the help of (6.16) we have proved that

$$
\left\|D^{l} u-D^{l} v\right\|_{L^{1}(\Omega)} \leq C \sum_{j}\left|E_{j}\right| \leq C \mathbf{M}(R) .
$$

Item 2 follows by combining (6.21) and (6.24). This completes the proof of Lemma 6.4. 
Using Lemma 6.4, we obtain the flat ${ }^{27}$ version of Theorem 1.3.

6.5 Lemma. Let $\Omega$ be a bounded open subset in $\mathbb{R}^{m}$ and let $M$ be the boundary of an $(m-k+1)$ rectifiable current $N$ in $\Omega$. Then there exists $u \in W^{k-1,1}\left(\Omega ; \mathbb{S}^{k-1}\right)$ such that $J u=M$ and $|u|_{W^{k-1,1}} \leq$ $C \mathbf{M}(N)$.

This follows from Lemma 6.4 and an iterative procedure which is exactly the same as the one in [2, proof of Theorem 5.6]; we omit the proof.

Proof of Theorem 1.3 when $p=1$. In what follows, we denote by $S_{r}^{m}$ the $m$-dimensional sphere $S(0, r) \subset \mathbb{R}^{m+1}$.

Let $\Omega:=\left\{x \in \mathbb{R}^{m+1} ; 1 / 2<|x|<3 / 2\right\}$. For every $l \geq 1, \emptyset \in C_{c}^{\infty}\left(\Lambda^{l} \Omega\right)$, and $r \in(1 / 2,3 / 2)$, we define $\omega_{>S_{r}^{m}} \in C^{\infty}\left(\Lambda^{l-1} \mathbb{S}^{m}\right)$ as follows:

$$
\varrho_{>S_{r}^{m}}(x)\left(\tau_{1}, \ldots, \tau_{l-1}\right)=\emptyset(r x)\left(\tau_{1}, \ldots, \tau_{l-1}, x\right), \quad \forall x \in \mathbb{S}^{m}, \tau_{j} \in T_{x} \mathbb{S}^{m}, j \in \llbracket 1, l-1 \rrbracket .
$$

If $T$ is an $(l-1)$-current in $\mathbb{S}^{m}$, then we define the $l$-current $T \times_{r}\left(\frac{1}{2}, \frac{3}{2}\right)$ in $\Omega$ through the formula

$$
\left\langle T \times_{r}\left(\frac{1}{2}, \frac{3}{2}\right), \emptyset\right\rangle:=\int_{1 / 2}^{3 / 2} r^{l-1}\left\langle T, \oplus_{>S_{r}^{m}}\right\rangle d r, \quad \forall \Subset \in C_{c}^{\infty}\left(\Lambda^{l} \Omega\right)
$$

Fix $\rho \in C_{c}^{\infty}(1 / 2,3 / 2)$ such that $\int \rho=1$. We introduce $X(x)=\frac{x}{|x|}, \forall x \in \mathbb{R}^{m+1} \backslash\{0\}$. For every $\zeta \in C^{\infty}\left(\Lambda^{m-k} \mathbb{S}^{m}\right)$, we define $\alpha=\alpha(\zeta) \in C_{c}^{\infty}\left(\Lambda^{m-k+1} \Omega\right)$ by

$$
\alpha(r x)=\rho(r) \widetilde{\zeta}(r x) \wedge d r, \quad \forall x \in \mathbb{S}^{m}, r \in(1 / 2,3 / 2),
$$

where $\widetilde{\zeta}=X^{\sharp} \zeta$. Then we claim that

$$
(d \alpha)_{>S_{r}^{m}}=\frac{1}{r^{m-k+1}} \rho(r) d \zeta .
$$

Here is a proof of this fact. We start from the identity $d \alpha=\frac{1}{r^{m-1}} \rho(r) X^{\sharp} d \zeta \wedge d r$. If $\tau_{1}, \ldots, \tau_{m-k+1} \epsilon$ $T_{x} \mathbb{S}^{m}$, then

$$
\begin{aligned}
d \alpha(r x)\left(\tau_{1}, \ldots, \tau_{m-k+1}, x\right) & =\rho(r)(d \widetilde{\zeta}(r x) \wedge d r)\left(\tau_{1}, \ldots, \tau_{m-k+1}, x\right) \\
& =\rho(r) d \widetilde{\zeta}(r x)\left(\tau_{1}, \ldots, \tau_{m-k+1}\right) d r(x) \\
& =\frac{1}{r^{m-k+1}} \rho(r) d \zeta(x)\left(\tau_{1}, \ldots, \tau_{m-k+1}\right) .
\end{aligned}
$$

Indeed, in (6.26), the next to the last equality relies on the fact that $d r\left(\tau_{j}\right)=0, \forall j \in \llbracket 1, m-k+1 \rrbracket$. In order to justify the last equality in (6.26), observe that $d X(r x)\left(\tau_{j}\right)=\frac{\tau_{j}}{r}\left(\right.$ since $\left\langle\tau_{j}, x\right\rangle=0$ ). This proves (6.25).

Let $N$ be an $(m-k+1)$-rectifiable current in $\mathbb{S}^{m}$. By Lemma 6.6 below, $K:=N \times_{r}\left(\frac{1}{2}, \frac{3}{2}\right)$ is an $(m-k+2)$-rectifiable current in $\Omega$, of mass comparable to the one of $N$. We apply Lemma 6.5 in $\Omega$ to the current $(-1)^{m-k+2} K$. Then there exists $v \in W^{k-1,1}\left(\Omega ; \mathbb{S}^{k-1}\right)$ such that $|v|_{W^{k-1,1}(\Omega)} \leq$ $C \mathbf{M}(N)$ and such that for every $\emptyset \in C_{c}^{\infty}\left(\Lambda^{m-k+1} \Omega\right)$,

$$
\int_{\Omega} d \omega \wedge v^{\sharp} \omega_{0}=\int_{1 / 2}^{3 / 2} r^{m-k+1}\left\langle N,(d \omega)_{>S_{r}^{m}}\right\rangle d r .
$$

In the case where $\emptyset=\alpha,(6.25)$ and (6.27) give

$$
\int_{\Omega} d \alpha \wedge v^{\sharp} \omega_{0}=\langle N, d \zeta\rangle .
$$

${ }^{27}$ I.e., with $\mathbb{S}^{m}$ replaced by $\Omega$. 
We next note the following identity: ${ }^{28}$ if we let

$$
h_{r}(x)=r x, v_{r}(x)=v(r x)=h_{r}^{\sharp} v(x), \quad \forall x \in \mathbb{S}^{m}, r \in\left(\frac{1}{2}, \frac{3}{2}\right),
$$

then

$$
d \alpha \wedge v^{\sharp} \omega_{0}(r x)=(-1)^{m-k+1} \rho(r) d r \wedge\left(d \widetilde{\zeta} \wedge v^{\sharp} \omega_{0}\right)_{\mathbb{S}_{r}^{m}}(r x) .
$$

Moreover,

$$
\int_{\mathbb{S}_{r}^{m}} d \widetilde{\zeta} \wedge v^{\sharp} \omega_{0}=\int_{\mathbb{S}^{m}} h_{r}^{\sharp}\left(d \widetilde{\zeta} \wedge v^{\sharp} \omega_{0}\right)=\int_{\mathbb{S}^{m}} d \zeta \wedge v_{r}^{\sharp} \omega_{0} .
$$

By (6.28), (6.29) and the Fubini theorem for forms, we have

$$
\langle N, d \zeta\rangle=(-1)^{m-k+1} \int_{1 / 2}^{3 / 2} \rho(r)\left(\int_{\mathbb{S}^{m}} d \zeta \wedge\left(\left(v_{r}\right)^{\sharp} \omega_{0}\right)\right) d r .
$$

The left-hand side of (6.31) does not depend on $\rho$. This proves that the function

$$
(1 / 2,3 / 2) \ni r \mapsto \int_{\mathbb{S}^{m}} d \zeta \wedge\left(\left(v_{r}\right)^{\sharp} \omega_{0}\right)
$$

is constant a.e.

On the other hand, for a.e. $r \in(1 / 2,3 / 2), v(r \cdot)$ belongs to $W^{k-1,1}\left(\mathbb{S}^{m} ; \mathbb{S}^{k-1}\right)$, and in addition we may find a positive measure subset $A \subset(1 / 2,3 / 2)$ such that $\left|v_{r}\right|_{W^{k-1,1}\left(\mathbb{S}^{m}\right)} \leq C \mathbf{M}(N)$ for every $r \in A$. By the above and the separability of $C^{1}\left(\Lambda^{m-k} \mathbb{S}^{m}\right)$, one can find $r \in A$ such that $u:=v(r \cdot)$ satisfy

$$
(-1)^{m-k+1} \int_{\mathbb{S}^{m}} d \zeta \wedge\left(u^{\sharp} \omega_{0}\right)=\langle N, d \zeta\rangle \quad, \quad \forall \zeta \in C^{\infty}\left(\Lambda^{m-k} \mathbb{S}^{m}\right) .
$$

This $u$ has all the required properties.

6.6 Lemma. Assume that $N$ is an $(l-1)$-rectifiable current in $\mathbb{S}^{m}$. Then $K:=N \times_{r}\left(\frac{1}{2}, \frac{3}{2}\right)$ is an l-rectifiable current in $\Omega$ and $\mathbf{M}(K) \sim \mathbf{M}(N)$.

Proof. Let $(\mathscr{M}, \xi, \theta)$ be the triple defining $N$. Define $\widetilde{\mathcal{M}}=\{r x ; x \in \mathscr{M}, 1 / 2<r<3 / 2\}$. Then clearly $\widetilde{\mathscr{M}}$ is an $l$-rectifiable part of $\Omega$, and for $\mathscr{H}^{l-1}$-a.e. $x \in \mathscr{M}$ and for every $r \in(1 / 2,3 / 2)$

$$
T_{r x} \widetilde{\mathscr{M}} \text { is spanned by the orthogonal spaces } T_{x} \mathscr{M} \text { and } \mathbb{R} x \text {. }
$$

We also set, with $r$ and $x$ as above, $\widetilde{\xi}(r x)=\xi(x), \widetilde{\theta}(r x)=\theta(x)$ and $X(r x)=\frac{x}{|x|}$.

With this notation, we have for $\emptyset \in C_{c}^{\infty}\left(\Lambda^{l} \Omega\right)$ :

$$
\langle K, \emptyset\rangle=\int_{1 / 2}^{3 / 2} r^{l-1} \int_{\mathscr{M}}\langle\widetilde{\xi}(r x), \varrho(r x)(\cdot, x)\rangle \theta(x) d \mathscr{H}^{l-1}(x) d r=\int_{\widetilde{M}}\langle\widetilde{\xi} \wedge X, \emptyset\rangle \widetilde{\theta} d \mathscr{P}^{l} .
$$

In view of (6.32), the $l$-current $K$ is given by the triple $(\widetilde{\mathcal{M}}, \widetilde{\xi} \wedge X, \widetilde{\theta})$, and we also have $\mathbf{M}(K) \sim$ $\mathbf{M}(N)$.

\footnotetext{
${ }^{28}$ Whose proof is similar to the one of (6.25) and left to the reader.
} 


\subsection{The case $k=2$ : geometrical approach}

Given an $(m-1)$-rectifiable current $N$ in $\mathbb{S}^{m}$, we have to construct a map $u \in \cap_{p \geq 1} W^{1 / p, p}\left(\mathbb{S}^{m} ; \mathbb{S}^{1}\right)$ such that $J u=\partial N$ and

$$
|u|_{W^{1 / p, p}}^{p} \leq C_{p} \mathbf{M}(N), \quad \forall p \geq 1 .
$$

This has to hold for every $p \geq 1$. In view of Lemma 4.5 , if $u \in W^{1 / p, p}\left(\mathbb{S}^{m} ; \mathbb{S}^{1}\right)$ for some $p>1$ and if (6.33) holds for this $p$, then for every $q>p$ we have $u \in W^{1 / q, q}\left(\mathbb{S}^{m} ; \mathbb{S}^{1}\right)$ and (6.33) holds. Therefore, it suffices to fix some $p_{1} \in(1,2)$ and to construct $u$ such that $u \in \cap_{1 \leq p \leq p_{1}} W^{1 / p, p}\left(\mathbb{S}^{m} ; \mathbb{S}^{1}\right)$ and

$$
|u|_{W^{1 / p, p}}^{p} \leq C_{p} \mathbf{M}(N), \quad \forall p \in\left[1, p_{1}\right] .
$$

We start by establishing the appropriate variant of Lemma 6.1.

6.7 Lemma. Let $E \subset \mathbb{R}^{m}$ be an oriented ( $\left.m-1\right)$-disc with center $x$ and radius $r$. Let $\sigma>0$. Then there exists $u: \mathbb{R}^{m} \rightarrow \mathbb{S}^{1}$ such that:

1. $u-1 \in \cap_{p \geq 1} W^{1 / p, p}\left(\mathbb{R}^{m}\right) \cap C^{\infty}\left(\mathbb{R}^{m} \backslash \partial E\right)$.

2. $J u=\partial E$.

3. $u=1$ in $\mathbb{R}^{m} \backslash \bar{B}(x, r)$ and

$$
\left|\left\{y \in \mathbb{R}^{m}: u(y) \neq 1\right\}\right| \leq \sigma .
$$

4. We have

$$
|u|_{W^{1 / p, p}}^{p} \leq C_{p}|E|, \forall p \geq 1 .
$$

Proof. In view of Lemma 6.1 and of its proof, it suffices to prove that the map $u$ constructed in that lemma (with $Y=1$ ) satisfies item 4 when $p>1$. By scaling, we may assume that the diameter of $E$ is 2 , and thus $u-1$ is supported in $\bar{B}(x, 1)$. Therefore, for $q \geq 1$ we have

$$
\|u-1\|_{W^{1, q}} \leq C_{q}\|\nabla u\|_{L^{q}} .
$$

Fix $p>1$ and consider $q \in(1, \min \{p, 2\})$. Define $r \in(q, \infty)$ by the formula

$$
\frac{p-1}{r}+\frac{1}{q}=1
$$

By Lemma 4.13 combined with (6.37), we have

$$
|u|_{W^{1 / p, p}}^{p} \leq C\|u-1\|_{W^{1, q}}\|u-1\|_{L^{r}}^{p-1} \leq C C_{q}\|\nabla u\|_{L^{q}}\|u-1\|_{L^{r}}^{p-1} .
$$

Item 3 implies that

$$
\|u-1\|_{L^{r}} \leq C \sigma^{1 / r} .
$$

On the other hand, estimate (6.5) with $l=1$ combined with the fact that $q<2$ leads to ${ }^{29}$

$$
\|\nabla u\|_{L^{q}}^{q} \leq C \sigma^{1-q} .
$$

We obtain item 4 by combining (6.38)-(6.41).

We next state and prove the remaining technical ingredients required to glue dipoles by keeping control of the $W^{1 / p, p}$ norm.

6.8 Lemma. Let $u, v: \mathbb{R}^{m} \rightarrow \mathbb{S}^{1}$ be such that $u-1, v-1 \in W^{1,1}$. Then $J(u v)=J u+J v$.

\footnotetext{
${ }^{29}$ The argument is the same as the one used in the proof of (6.6).
} 
Proof. This follows by combining the definition (1.7) with the identity

$(u v)^{\sharp} \omega_{0}=u^{\sharp} \omega_{0}+v^{\sharp} \omega_{0}$.

6.9 Lemma. Let $u, v: \mathbb{R}^{m} \rightarrow \mathbb{S}^{1}$ be such that $u-1, v-1 \in W^{1,1} \cap W^{1 / p, p}\left(\mathbb{R}^{m}\right)$ for some $p>1$. Then

1. $|u v-u|_{W^{1,1}} \leq|v|_{W^{1,1}}+2|u|_{W^{1,1}([v \neq 1])}$.

2. $|u v-u|_{W^{1 / p, p}} \leq|v|_{W^{1 / p, p}}+2\left(\iint_{v(x) \neq 1} \frac{|u(x)-u(y)|^{p}}{|x-y|^{m+1}} d x d y\right)^{1 / p}$.

Proof. Item 1 follows from

$|\nabla(u v-u)| \leq|\nabla v|+|v-1||\nabla u| \leq|\nabla v|+2 \mathbb{1}_{[v \neq 1]}|\nabla u|$.

Item 2 is a consequence of

$$
\begin{aligned}
|(u(x) v(x)-u(x))-(u(y) v(y)-u(y))| & =|u(y)(v(x)-v(y))+(u(x)-u(y))(v(x)-1)| \\
& \leq|v(x)-v(y)|+|v(x)-1||u(x)-u(y)| \\
& \leq|v(x)-v(y)|+2 \mathbb{1}_{[v(x) \neq 1]}|u(x)-u(y)| .
\end{aligned}
$$

6.10 Lemma. Let $1<q<2$ and $u: \mathbb{R}^{m} \rightarrow \mathbb{S}^{1}$ be such that $u-1 \in W^{1, q}\left(\mathbb{R}^{m}\right)$. Consider the $L^{q}$ modulus of continuity of $\nabla u$ :

$$
\omega(\delta):=\sup \left\{\|\nabla u\|_{L^{q}(A)} ; A \subset \mathbb{R}^{m},|A| \leq \delta\right\} .
$$

Then there are constants $C_{p}, 1<p \leq q$, independent of $u$, such that

$$
I_{p}(A):=\iint_{x \in A} \frac{|u(x)-u(y)|^{p}}{|x-y|^{m+1}} d x d y \leq C_{p}\left(|A|+[\omega(|A|)]^{p}|A|^{(q-p) / q}\right), \quad \forall A \subset \mathbb{R}^{m} .
$$

In particular, given $\varepsilon>0$ there exists some $\delta>0$ such that, if $|A| \leq \delta$, then $I_{p}(A) \leq C_{p} \varepsilon, \forall p \in$ $(1, q]$.

Proof. By a standard approximation argument, we may assume $u$ smooth and bounded by 1 (but not necessarily circle-valued).

We split $I_{p}(A)=J_{p}(A)+K_{p}(A)$, where

$$
J_{p}(A)=\iint_{x \in A,|y-x| \geq 1} \ldots, K_{p}(A)=\iint_{x \in A,|y-x|<1} \ldots
$$

On the one hand, we have

$$
J_{p}(A) \leq 2^{p}|A| \int_{|z| \geq 1} \frac{1}{|z|^{m+1}} d z=C_{p}|A| .
$$

On the other hand, the inequality

$$
|u(x+z)-u(x)|^{p} \leq|z|^{p} \int_{0}^{1}|\nabla u(x+t z)|^{p} d t
$$

leads to

$$
\begin{aligned}
K_{p}(A) & \leq \int_{0}^{1} \int_{x \in A} \int_{z \in B^{m}} \frac{|\nabla u(x+t z)|^{p}}{|z|^{m-p+1}} d z d x d t \\
& \leq \int_{0}^{1} t^{1-p} \int_{w \in B^{m}} \frac{1}{|w|^{m-p+1}} \int_{x \in w+A}|\nabla u(x)|^{p} d x d z d t \\
& \leq C_{p} \sup _{w}\|\nabla u\|_{L^{p}(w+A)}^{p} \leq C_{p}[\omega(|A|)]^{p}|A|^{(q-p) / q} .
\end{aligned}
$$

We obtain (6.42) by combining (6.43) with (6.44). 
6.11 Lemma. Let $1<q<2$. Consider a finite collection $\left(E_{j}\right)$ of oriented $(m-1)$-discs of centers $x_{j}$ and radii $r_{j}$ in $\mathbb{R}^{m}$, such that the balls $\bar{B}^{m}\left(x_{j}, 2 r_{j}\right)$ are pairwise disjoint. Let $u: \mathbb{R}^{m} \rightarrow \mathbb{S}^{1}$ be such that $u-1 \in W^{1, q}\left(\mathbb{R}^{m}\right)$. Let $\varepsilon>0$. Then there exists a map $v: \mathbb{R}^{m} \rightarrow \mathbb{S}^{1}$ such that:

1. $v-1 \in W^{1, r}\left(\mathbb{R}^{m}\right), \forall r \in[1,2)$.

2. $J v=\sum \partial E_{j}$.

3. $|v|_{W^{1 / p, p}}^{p} \leq C_{p} \sum\left|E_{j}\right|, \forall p \in[1,2]$.

4. $|u v-u|_{W^{1 / p, p}} \leq|v|_{W^{1 / p, p}}+C_{p} \varepsilon^{1 / p}, \forall p \in[1, q]$.

Proof. Associate to each $j$ and $\sigma>0$ a map $v_{j, \sigma}$ as in Lemma 6.7. We will take $v=\prod v_{j, \sigma}$ for a convenient $\sigma$. Note that, by the proof of Lemma 6.7, $v$ satisfies item 1. On the other hand, item 2 follows by combining Lemma 6.72 with Lemma 6.8 .

We next turn to 3 . Let $A_{j, \sigma} \subset B\left(x_{j}, r_{j}\right)$ denote the set of measure $\leq \sigma$ in which $v_{j, \sigma} \neq 1$. Set $B_{j}:=B\left(x_{j}, 2 r_{j}\right)$. Let $C:=\mathbb{R}^{m} \backslash \cup_{j} B_{j}$. Set also $w_{p}(x, y)=\frac{|v(x)-v(y)|^{p}}{|x-y|^{m+1}}$ and $f(x, y)=\frac{1}{|x-y|^{m+1}}$. The identity

$$
\begin{aligned}
|v|_{W^{1 / p, p}}^{p}= & \sum_{j} \iint_{B_{j} \times\left(B_{j} \cup C\right)} w_{p}+\sum_{j} \iint_{A_{j, \sigma} \times\left(\cup_{k \neq j} B_{k}\right)} w_{p}+\sum_{j} \iint_{\left(B_{j} \backslash A_{j, \sigma}\right) \times\left(\cup_{k \neq j} A_{k, \sigma}\right)} w_{p} \\
& +\iint_{C \times\left(\cup_{j} A_{j, \sigma}\right)} w_{p}
\end{aligned}
$$

leads to the estimate

$$
|v|_{W^{1 / p, p}}^{p} \leq \sum_{j}\left|v_{j, \sigma}\right|_{W^{1 / p, p}}^{p}+2^{p+1} \sum_{j} \underbrace{\iint_{A_{j, \sigma} \times\left(C \cup \cup_{k \neq j} B_{k}\right)} f}_{L_{j, \sigma}} .
$$

We obtain item 3 via Lemma 6.74 combined with the fact that $L_{j, \sigma} \rightarrow 0$ as $\sigma \rightarrow 0$.

Finally, item 4 with $p>1$ follows from Lemma 6.10 combined with Lemma 6.92 and with the fact that the set $[v \neq 1]$ can be chosen arbitrarily small. The case $p=1$ follows immediately from Lemma 6.91.

First proof of Theorem 1.3 in $\mathbb{R}^{m}$ for $k=2$. As explained at the beginning of this section, we fix $p_{1} \in(1,2)$. Consider the oriented ( $\left.m-1\right)$-discs $E_{j, l}$ as in Corollary 6.3. Let $u_{0} \equiv 1$. By Lemma 6.11, for every sequence $\left(\varepsilon_{l}\right)$ of positive numbers we may construct inductively maps $u_{l}$ such that $u_{l}-1 \in W^{1, r}\left(\mathbb{R}^{m}\right), \forall 1 \leq r<2, J u_{l}=\sum_{j} \partial E_{j, l},\left|u_{l}\right|_{W^{1 / p, p}}^{p} \leq C_{p} \sum_{j}\left|E_{j, l}\right|, \forall p \in[1,2]$, and

$$
\left|u_{0} \ldots u_{l}-u_{0} \ldots u_{l-1}\right|_{W^{1 / p, p}} \leq\left|u_{l}\right|_{W^{1 / p, p}}+C_{p} \varepsilon_{l}^{1 / p}, \quad \forall p \in\left[1, p_{1}\right] .
$$

Moreover,

$$
\left\|u_{0} \ldots u_{l}-1\right\|_{L^{p\left(\mathbb{R}^{m}\right)}}^{p} \leq C \sum_{l} \sum_{j}\left|E_{j, l}\right|^{m /(m-1)} \leq C\left(\sum_{l} \sum_{j}\left|E_{j, l}\right|\right)^{m /(m-1)} .
$$

Using Corollary 6.32 and choosing $\varepsilon_{l}=2^{-l} \mathbf{M}(N)$, we find that the product $\prod_{j} u_{j}$ converges in $W_{l o c}^{1 / p, p}\left(\mathbb{R}^{m} ; \mathbb{S}^{1}\right), \forall p \in\left[1, p_{1}\right]$, to some $u$ such that $u-1 \in W^{1 / p, p}\left(\mathbb{R}^{m}\right), J u=\partial N$ and $|u|_{W^{1 / p, p}}^{p} \leq$ $C_{p} \mathbf{M}(N)$.

Proof of Theorem 1.3 for $k=2$. Let $N$ be an $(m-1)$-rectifiable current in $\mathbb{S}^{m}$. Let $\Omega=\{x \in$ $\left.\mathbb{R}^{m+1} ; 1 / 2<|x|<3 / 2\right\}$ and $K=N \times_{r}\left(\frac{1}{2}, \frac{3}{2}\right)$. By the above, there exists a map $v \in \cap_{p \geq 1} W^{1 / p, p}\left(\Omega ; \mathbb{S}^{1}\right)$ such that $J v=(-1)^{m} \partial K$ and

$$
|v|_{W^{1 / p, p}}^{p} \leq C_{p} \mathbf{M}(K) \leq C_{p}^{\prime} \mathbf{M}(N), \quad \forall p \geq 1 .
$$


Let $v_{r}=v(r \cdot), 1 / 2<r<3 / 2$. We have the following inequality: ${ }^{30}$

$$
\int_{1 / 2}^{3 / 2}\left|v_{r}\right|_{W^{s, p}\left(\mathbb{S}^{m}\right)}^{p} d r \leq C_{s, p}|v|_{W^{s, p}(\Omega)}^{p}, \quad \forall s \geq 0, \forall p \in[1, \infty) .
$$

We will also use the following elementary fact: if $\left(f_{n}\right)_{n \geq 0}$ is a sequence of non-negative integrable functions in $(a, b)$, then there exists a set $A \subset(a, b)$ of measure $\geq(b-a) / 2$ such that

$$
f_{n}(t) \leq 2^{n+2} f_{a}^{b} f_{n}, \quad \forall n \in \mathbb{N}, \forall t \in A
$$

The proof of Theorem 1.3 for $p=1$ implies that

$$
J v_{r}=\partial N \text { for a.e. } r \in(1 / 2,3 / 2) .
$$

Let $p_{0}=1$ and consider a sequence $\left(p_{n}\right)_{n \geq 1} \subset(1, \infty)$ such that $p_{n} \backslash 1$. By (6.46)-(6.49), ${ }^{31}$ there exists some $r$ such that $u:=v_{r}$ satisfies:

1. $u \in \cap_{n \geq 0} W^{1 / p_{n}, p_{n}}\left(\mathbb{S}^{m} ; \mathbb{S}^{1}\right)$.

2. $J u=\partial N$.

3. $|u|_{W^{1 / p_{n}, p_{n}}}^{p_{n}} \leq K_{n} \mathbf{M}(N), \forall n \in \mathbb{N}$.

By combining item 3 with Lemma 4.5, we find that $u$ has all the properties required by Theorem 1.3.

\subsection{The case $k=2$ : analytical approach}

As explained in the previous section, it suffices to establish Theorem 1.3 in a bounded domain $\Omega$ in an Euclidean space. The case of maps defined in $\mathbb{S}^{m}$ follows from this special case. Let $\Omega \subset \mathbb{R}^{m}$ be smooth bounded. We consider a slightly larger smooth bounded open set, $U$, whose role is to enable the definition of a convenient class of almost smooth maps. Let $u \in W^{1,1}\left(\Omega ; \mathbb{S}^{1}\right)$. By reflections across $\partial \Omega$, we may extend $u$ to $U$, with norm control. Using, in $U$, the density result of Bethuel and Zheng [8, proof of Theorem 1], we obtain the following: the class

$$
\mathscr{R}=\left\{u \in C^{\infty}(\Omega \backslash \Sigma(u)) \cap W^{1,1}\left(\Omega ; \mathbb{S}^{1}\right) ;\left|D^{l} u(x)\right| \leq \frac{C_{l}(u)}{\left[\delta_{u}(x)\right]^{l}}, \forall l \in \mathbb{N}\right\}
$$

is dense in $W^{1,1}(\Omega)$. Here, $\Sigma(u)$ is a smooth $(m-2)$-submanifold of $U$, and $\delta_{u}$ denotes the distance to $\Sigma(u)$.

It will be convenient to consider the more general class $\widetilde{\mathscr{R}}$, defined as $\mathscr{R}$, except that we allow $\Sigma(u)$ to be a finite union of $(m-2)$-submanifolds of $U$. It is clear that $\widetilde{\mathscr{R}}$ is an algebra; in particular, the product of two maps in $\mathscr{R}$ belongs to $\widetilde{\mathscr{R}}$. Using the density of the class $\mathscr{R}$ and a straightforward Cauchy sequences argument, we obtain the following result.

6.12 Lemma. Let $u \in W^{1,1}\left(\Omega ; \mathbb{S}^{1}\right)$. Then there exists a sequence $\left(v_{j}\right)_{j \geq 0} \subset \widetilde{\mathscr{R}}$ such that:

1. $v_{1} \ldots v_{j} \rightarrow u$ in $W^{1,1}$ as $j \rightarrow \infty$.

2. $\left\|\nabla v_{j}\right\|_{L^{1}} \leq 2^{-j}\|\nabla u\|_{L^{1}}, \forall j \geq 0$.

By the above, we have $J u=\sum J v_{j}$, the series being convergent in $\left(W^{1, \infty}\left(\Lambda^{m-2} \Omega\right)\right)^{*}$. We may now explain our strategy for proving Theorem 1.3. Let $N$ be a rectifiable $(m-1)$-current in $\Omega$. By [2, Theorem 5.6], we may find some $u \in W^{1,1}\left(\Omega ; \mathbb{S}^{1}\right)$ such that $J u=\partial N$ and $\|\nabla u\|_{L^{1}} \leq C \mathbf{M}(N)$. Now comes the main idea (Lemma 6.15 below): we replace $v_{j}$ by some $w_{j}$ such that $J w_{j}=J v_{j}$ and such that the product $\prod_{j} w_{j}$ converges in $W^{1 / p, p}$ for every $p \geq 1$, with norm control. Note the difference with Lemma 6.122 , which provides control only for the $W^{1,1}$ norm. Once Lemma 6.15 is established, we prove that the map $w=\prod_{j} w_{j}$ satisfies $J w=\partial N$ and all the required estimates.

We now proceed to the proofs. We start by recalling the following result [2, Corollary 3.9].

${ }^{30}$ For the flat case, see [1, Example 7.27], [1, Lemma 7.44]. The argument there adapts to manifolds.

${ }^{31}$ Inequality (6.48) is applied with $(a, b)=(1 / 2,3 / 2)$ and $f_{n}(r)=\left|v_{r}\right|_{W^{1 / p_{n}, p_{n}\left(\mathbb{S}^{m}\right)}}^{p_{n}}$. 
6.13 Lemma. Let $\varphi: \mathbb{S}^{1} \rightarrow \mathbb{S}^{1}$ be Lipschitz. Let $u \in W^{1,1}\left(\Omega ; \mathbb{S}^{1}\right)$. Then $J(\varphi \circ u)=\operatorname{deg} \varphi J u$.

For $\varepsilon \in(0,2 \pi)$ and $\theta \in \mathbb{R}$, we let

$$
\varphi_{\varepsilon, \theta}: \mathbb{S}^{1} \rightarrow \mathbb{S}^{1}, \varphi_{\varepsilon, \theta}\left(e^{\imath \psi}\right)=\left\{\begin{array}{ll}
e^{2 \imath \pi(\psi-\theta) / \varepsilon}, & \text { if } \psi \in[\theta, \theta+\varepsilon] \\
1, & \text { if } \psi \in[\theta+\varepsilon, \theta+2 \pi]
\end{array} .\right.
$$

We collect some straightforward properties of $\varphi_{\varepsilon, \theta}$.

6.14 Lemma. We have:

1. $\varphi_{\varepsilon, \theta}$ is Lipschitz of constant $2 \pi / \varepsilon$ and of degree 1 .

2. If $z_{1}, z_{2} \in \mathbb{S}^{1}$ and $p \in[1, \infty)$, then

$$
\int_{0}^{2 \pi}\left|\varphi_{\varepsilon, \theta}\left(z_{1}\right)-\varphi_{\varepsilon, \theta}\left(z_{2}\right)\right|^{p} d \theta \leq C_{p} \min \left\{\varepsilon, \frac{\left|z_{1}-z_{2}\right|^{p}}{\varepsilon^{p-1}}\right\}
$$

Proof. Only (6.50) requires a proof. Let $I_{\varepsilon}(z)=\left\{w \in \mathbb{S}^{1} ; d(z, w)<\varepsilon\right\}$; here, $d$ denotes the geodesic distance in $\mathbb{S}^{1}$. If $e^{\imath \theta} \notin I_{\varepsilon}\left(z_{1}\right) \cup I_{\varepsilon}\left(z_{2}\right)$, then $\varphi_{\varepsilon, \theta}\left(z_{1}\right)=\varphi_{\varepsilon, \theta}\left(z_{2}\right)=1$; therefore, the integral in (6.50) has to be considered only over $I_{\varepsilon}\left(z_{1}\right) \cup I_{\varepsilon}\left(z_{2}\right)$. On the other hand, we have

$$
\left|\varphi_{\varepsilon, \theta}\left(z_{1}\right)-\varphi_{\varepsilon, \theta}\left(z_{2}\right)\right|^{p} \leq \min \left\{2^{p}, \frac{2^{p} \pi^{p} d^{p}\left(z_{1}, z_{2}\right)}{\varepsilon^{p}}\right\} .
$$

We obtain the conclusion by integrating (6.51) over $I_{\varepsilon}\left(z_{1}\right) \cup I_{\varepsilon}\left(z_{2}\right)$.

6.15 Lemma. Let $v \in \widetilde{\mathscr{R}}$ and $\delta>0$. Then there are constants $C_{p}, \forall p \in[1, \infty)$, independent of $v$ and $\delta$, and a Lipschitz map $\varphi: \mathbb{S}^{1} \rightarrow \mathbb{S}^{1}$ of degree 1 such that $w:=\varphi \circ v$ satisfies:

1. $|w|_{W^{1 / p, p}}^{p} \leq C_{p}\|\nabla v\|_{L^{1}}$.

2. $|\{w \neq 1\}| \leq \delta$.

A word about the conclusion of Lemma 6.15. Unlike the case $k \geq 3$, one cannot rely on the Gagliardo-Nirenberg inequalities. Indeed, the inclusion $W^{1,1} \cap L^{\infty} \subset W^{1 / p, p}$ is wrong for every $p>1 .{ }^{32}$ Lemma 6.15 essentially asserts the following unexpected result: let $v: \Omega \rightarrow \mathbb{S}^{1}$ be slightly better then $W^{1,1}$. Then we may rearrange the values of $v$ (that is, consider $w=\varphi(v)$, with $\varphi$ a smooth homeomorphism of $\mathbb{S}^{1}$ into itself) such that the new map $w$ is controlled in $W^{1 / p, p}$ for every $p>1$, with a control depending only on $p$ and $\|\nabla v\|_{L^{1}}$, but not on higher order norms of $v .^{33}$ By Lemma 6.13 and Lemma 6.14, we have $J w=J v$. Though we state and prove Lemma 6.15 for circle-valued maps, it will be transparent from the proof that the target could be any sphere.

Proof. Let $\varepsilon \in(0,2 \pi)$ and $w_{\varepsilon, \theta}:=\varphi_{\varepsilon, \theta} \circ v$. We will establish, for sufficiently small $\varepsilon$, the following estimates:

$$
\begin{aligned}
& \int_{0}^{2 \pi}\left|\left\{w_{\varepsilon, \theta} \neq 1\right\}\right| d \theta=\varepsilon|\Omega| . \\
& \int_{0}^{2 \pi}\left|w_{\varepsilon, \theta}\right|_{W^{1 / p, p}}^{p} d \theta \leq K_{p}\left(C(v) \sqrt{\varepsilon}+\|\nabla v\|_{L^{1}}\right), \quad \forall p \in[1, \infty) .
\end{aligned}
$$

\footnotetext{
${ }^{32}$ This is well-known to the experts. Here is an explicit example. Let $\rho=\mathbb{1}_{[-1 / 2,1 / 2]}$. If $A \subset \mathbb{R}$ is an interval, let $\widetilde{A}$ define the interval with the same center as $A$ and twice longer than $A$. Consider, in $(0,1)$ and for sufficiently large $j$, intervals $A_{j}$ such that the $\widetilde{A_{j}}$ 's are mutually disjoint and $\left|A_{j}\right|=1 / j^{2}$. Let $\varepsilon_{j}=\frac{1}{j^{2}} e^{-e^{j}}$. Then $u:=\sum \frac{1}{j^{2}} \mathbb{1}_{A_{j}} * \rho_{\varepsilon_{j}}$ belongs to $W^{1,1}(0,1)$, but to none of the spaces $W^{1 / p, p}(0,1)$ with $p>1$.

${ }^{33}$ The map $\varphi$ considered in the proof of Lemma 6.15 is not a diffeomorphism, but it is easy to slightly modify it and transform it into a diffeomorphism.
} 
Taking these two estimates for granted, we proceed as follows: for small $\varepsilon$ we have $C(v) \sqrt{\varepsilon} \leq$ $\|\nabla v\|_{L^{1}}$ and $\varepsilon<\pi \delta /|\Omega|$. Consider such an $\varepsilon$. As at the end of the Section 6.2, estimate (6.52) and estimate (6.53) applied along a sequence $p_{n} \backslash 1$ combined with estimate (6.48) and with the Gagliardo-Nirenberg inequalities imply that the conclusions of Lemma 6.15 hold with $w=\varphi_{\varepsilon, \theta}$ for appropriate $\varepsilon$ and $\theta$.

Step 1. Proof of (6.52). For $z \in \mathbb{S}^{1}$, the $\operatorname{set}\left\{\theta ; w_{\varepsilon, \theta}(z) \neq 1\right\}$ has measure $\varepsilon$, and thus

$$
\int_{0}^{2 \pi}\left|\left\{w_{\varepsilon, \theta} \neq 1\right\}\right| d \theta=\int_{\Omega}\left|\left\{\theta ; w_{\varepsilon, \theta}(v(x)) \neq 1\right\}\right| d x=\varepsilon|\Omega| .
$$

Step 2. Proof of (6.53) when $p=1$. We will actually prove that the term $C(v) \sqrt{\varepsilon}$ is not needed for $p=1$. As in Step 1, using the fact that $\left|\varphi_{\varepsilon, \theta}^{\prime}(z)\right|=2 \pi / \varepsilon$ if $\varphi_{\varepsilon, \theta}(z) \neq 1$ and that $\varphi_{\varepsilon, \theta}^{\prime}(z)=0$ a.e. otherwise, we find that

$$
\int_{0}^{2 \pi}\left\|\nabla w_{\varepsilon, \theta}\right\|_{L^{1}} d \theta=2 \pi\|\nabla v\|_{L^{1}}
$$

Step 3. Proof of (6.53) when $p>1$. Using a second order Taylor expansion, it is easy to see that the following inequality holds uniformly in $x, y$ and $\varepsilon<1 / 4$ :

$$
|v(x)-v(y)| \leq|\nabla v(x)||x-y|+\frac{K(v)}{\delta_{v}^{2}(x)}|x-y|^{2} \quad \text { if }|y-x| \leq \sqrt{\varepsilon} \delta_{v}(x) .
$$

On the other hand, we clearly have

$$
\int_{\Omega} \frac{1}{\left[\delta_{v}(x)\right]^{q}} d x<\infty, \quad \forall q \in[1,2) ;
$$

here we see why we have to replace $\Omega$ by $U$ in the definition of $\widetilde{\mathscr{R}}$. In particular, (6.56) combined with the definition of $\widetilde{\mathscr{R}}$ implies that

$$
w_{\varepsilon, \theta} \in W^{1, q}(\Omega), \quad \forall q \in[1,2), \varepsilon, \theta \in(0,2 \pi) .
$$

We split

$$
I:=\int_{0}^{2 \pi}\left|w_{\varepsilon, \theta}\right|_{W^{1 / p, p}}^{p} d \theta=\int_{0}^{2 \pi} \int_{\Omega} \int_{\Omega} \frac{\left|w_{\varepsilon, \theta}(x)-w_{\varepsilon, \theta}(y)\right|^{p}}{|x-y|^{m+1}} d x d y d \theta=I_{1}+\ldots+I_{4},
$$

where $I_{j}$ corresponds to the integration over $A_{j}, \forall j \in \llbracket 1,4 \rrbracket$, and:

$$
\begin{aligned}
& A_{1}=\left\{(x, y) \in \Omega^{2} ;|x-y| \geq \sqrt{\varepsilon} \delta_{v}(x)\right\}, \\
& A_{2}=\left\{(x, y) \in \Omega^{2} ; \frac{\varepsilon}{|\nabla v(x)|}<|x-y|<\sqrt{\varepsilon} \delta_{v}(x)\right\}, \\
& A_{3}=\left\{(x, y) \in \Omega^{2} ; \sqrt{\frac{\varepsilon}{K(v)}} \delta_{v}(x)<|x-y|<\sqrt{\varepsilon} \delta_{v}(x)\right\}, \\
& A_{4}=\Omega^{2} \backslash\left(A_{1} \cup A_{2} \cup A_{3}\right) .
\end{aligned}
$$

Step 4. Estimate of $I_{1}$ and $I_{3}$. By (6.50) and (6.56), we have

$$
I_{1} \leq C_{p} \varepsilon \iint_{A_{1}} \frac{1}{|y-x|^{m+1}} d y d x \leq C_{p} \sqrt{\varepsilon} \int_{\Omega} \frac{1}{\delta_{v}(x)} d x \leq C_{p} C(v) \sqrt{\varepsilon} .
$$

By the same argument, we obtain

$$
I_{3} \leq C_{p} C(v) \sqrt{\varepsilon K(v)}
$$


Step 5. Estimate of $I_{2}$. By (6.50), we have

$$
I_{2} \leq C_{p} \varepsilon \iint_{A_{2}} \frac{1}{|y-x|^{m+1}} d y d x \leq C_{p}\|\nabla v\|_{L^{1}}
$$

Step 6. Estimate of $I_{4}$. By combining (6.50) with (6.55), we find that

$$
I_{4} \leq \frac{C_{p}}{\varepsilon^{p-1}} \underbrace{\iint_{A_{4}} \frac{|\nabla v(x)|^{p}}{|y-x|^{m+1-p}} d y d x}_{I_{5}}+\frac{C_{p} K^{p}(v)}{\varepsilon^{p-1}} \underbrace{\iint_{A_{4}} \frac{1}{\delta_{v}^{2 p}(x)|y-x|^{m+1-2 p}} d y d x}_{I_{6}} .
$$

Since $|y-x| \leq \frac{\varepsilon}{|\nabla v(x)|}$ when $(x, y) \in A_{4}$, we find that

$$
I_{5} \leq C_{p} \varepsilon^{p-1}\|\nabla v\|_{L^{1}}
$$

Similarly, the fact that $|y-x| \leq \sqrt{\frac{\varepsilon}{K(v)}} \delta_{v}(x)$ in $A_{4}$ leads to

$$
I_{6} \leq C_{p}\left(\frac{\varepsilon}{K(v)}\right)^{p-1 / 2} \int_{\Omega} \frac{1}{\delta_{v}(x)} d x .
$$

We complete the proof of (6.53) when $p>1$ by combining (6.58)-(6.64).

6.16 Remark. The above construction is indeed, as stated in the introduction, a dipole construction. Indeed, the support of $w-1$ can be chosen arbitrarily small, while $J w$ is prescribed.

Lemma 6.15 leads to the following straightforward variant of Lemma 6.11, whose proof is left to the reader.

6.17 Lemma. Let $1<q<2$ and let $u \in W^{1, q}(\Omega)$. Let $\varepsilon>0$ and $v \in \widetilde{\mathscr{R}}$. Then there exists $w \in \widetilde{\mathscr{R}}$ such that:

1. $J w=J v$.

2. $|w|_{W^{1 / p, p}}^{p} \leq C_{p}\|\nabla v\|_{L^{1}}, \forall p \in[1, \infty)$.

3. $|u w-u|_{W^{1 / p, p}} \leq|w|_{W^{1 / p, p}}+C_{p} \varepsilon^{1 / p}, \forall p \in[1, q]$.

Second proof of Theorem 1.3 when $\Omega \subset \mathbb{R}^{m}$ is bounded. Let $N$ be an $(m-1)$-rectifiable current in $\Omega$. Let $u \in W^{1,1}\left(\Omega ; \mathbb{S}^{1}\right)$ be such that $J u=\partial N$ and $\|\nabla u\|_{L^{1}} \leq C \mathbf{M}(N)$. Let $v_{j}$ be as in Lemma 6.12. By applying inductively Lemma $6.17,{ }^{34}$ we construct a sequence $\left(w_{j}\right)$ such that $J w_{j}=$ $J v_{j},\left|w_{j}\right|_{W^{1 / p, p}}^{p} \leq C_{p} 2^{-j} \mathbf{M}(N)$ and

$$
\left|w_{1} \ldots w_{j+1}-w_{1} \ldots w_{j}\right|_{W^{1 / p, p}} \leq\left|w_{j+1}\right|_{W^{1 / p, p}}+C_{p}\left(2^{-j} \mathbf{M}(N)\right)^{1 / p}, \quad \forall p \in[1,3 / 2] .
$$

By combining the above with Lemma 4.5, it is easy to see that the product $\prod_{j} w_{j}$ converges in $W^{1 / p, p}\left(\Omega ; \mathbb{S}^{1}\right), \forall p \in[1, \infty)$, to some $w$ that has all the properties required in Theorem 1.3.

${ }^{34}$ Here, we use the fact that $\widetilde{\mathscr{R}} \subset W^{1, q}(\Omega), \forall q \in[1,2), c f(6.57)$. 


\section{$7 \quad$ Proof of Lemma 5.1}

Let $\left(U_{j}\right)_{1 \leq j \leq 2}$ be an open covering of $\mathbb{S}^{m}$ by domains of local charts, and let $\varphi_{j}: \bar{U}_{j} \rightarrow \bar{B}^{m}$ be the corresponding smooth diffeomorphisms. We denote by $\overline{\varphi_{j}}$ the corresponding charts for $\mathbb{S}^{m} \times[0, \infty): \overline{\varphi_{j}}(x, t)=\left(\varphi_{j}(x), t\right) \in \mathbb{R}^{m} \times \mathbb{R}$. Let $\left(\theta_{j}\right)_{1 \leq j \leq 2}$ be a partition of the unit subordinate to the covering $\left(U_{j}\right)_{1 \leq j \leq 2}$ of $\mathbb{S}^{m}$. Thus $\theta_{j}$ is compactly supported in $U_{j}$ and $\theta_{1}+\theta_{2}=1$. For $1 \leq j \leq 2$, we define $h_{j}:=\varphi_{j \sharp}\left(\theta_{j} u\right)$, which is compactly supported in $B^{m}$ with values into $\bar{B}^{k}$. Assume for the moment that we can construct a map $w_{j}: \mathbb{R}^{m} \times[0, \infty) \rightarrow \bar{B}^{k}$ which satisfies all the properties enumerated in Lemma 5.1 when $u$ is replaced by $h_{j}$ and $\mathbb{S}^{m}$ by $B^{m}$. Let $\eta \in C_{c}^{\infty}\left(B^{m}\right)$ such that $\eta=1$ on $\cup_{j} \varphi_{j}\left(\operatorname{supp} \theta_{j}\right)$. We then define $v(x, t):=\sum_{j} \overline{\varphi_{j}}\left(\eta w_{j}\right)=\sum_{j} \eta\left(\varphi_{j}(x)\right) w_{j}\left(\varphi_{j}(x), t\right)$. Clearly, $v \in C^{\infty}\left(\mathbb{S}^{m} \times(0, \infty)\right)$. If, in addition, $u$ is smooth, then

$$
v(x, 0)=\sum_{j} \eta\left(\varphi_{j}(x)\right) w_{j}\left(\varphi_{j}(x), 0\right)=\sum_{j} \eta\left(\varphi_{j}(x)\right) h_{j}\left(\varphi_{j}(x)\right)=\sum_{j} \eta\left(\varphi_{j}(x)\right) \theta_{j}(x) u(x) .
$$

Since $\eta\left(\varphi_{j}(x)\right)=1$ when $\theta_{j}(x) \neq 0$, we find that $v(x, 0)=u(x)$. We shall prove that for $j=1,2$ and $T>0$ :

$$
\left\|w_{j}\right\|_{L^{p}\left(\mathbb{R}^{m} \times(0, T)\right)} \leq C_{T}\left\|h_{j}\right\|_{L^{p}\left(\mathbb{R}^{m}\right)},\left|w_{j}\right|_{W^{k / p, p}\left(\mathbb{R}^{m} \times(0, \infty)\right)} \leq C\left|h_{j}\right|_{W^{(k-1) / p, p}\left(\mathbb{R}^{m}\right)} .
$$

Moreover, we will see that if $\|u\|_{L^{\infty}} \leq 1$, then

$$
\left|w_{j}(x, t)\right|+\left|d_{x} w_{j}(x, t)\right|+\left|\partial_{t} w_{j}(x, t)\right| \leq \frac{C}{t} .
$$

The chain rule easily implies that this $v$ satisfies estimate (5.1) when the seminorms are replaced by norms. By applying the argument leading to Corollary 4.3, we finds (5.1) holds also in its original form (with seminorms). ${ }^{35}$

If (7.1) holds true, then we obtain (5.2) as follows.

$$
\begin{aligned}
\int_{\mathbb{S}^{m}}|v(x, \cdot)|_{\left.W^{k / p, p}((0, \infty))\right)}^{p} & \leq C \sum_{j} \int_{\mathbb{R}^{m}}\left|w_{j}(x, \cdot)\right|_{W^{k / p, p}((0, \infty))}^{p} \\
& \leq C \sum_{j}\left|h_{j}\right|_{W^{(k-1) / p, p}\left(\mathbb{R}^{m}\right)}^{p} \leq C\|u\|_{W^{(k-1) / p, p}\left(\mathbb{S}^{m}\right)}^{p}
\end{aligned}
$$

We obtain (5.2) by applying (7.3) to $u-f_{\mathbb{S}^{m}} u$.

Finally, Lemma 5.1 item 5 is a direct consequence of (7.2) above.

In conclusion of the above discussion, we only need to prove Lemma 5.1 when $\mathbb{S}^{m}$ is replaced by $B^{m}$ and $u$ is compactly supported. In this case, we will prove that the standard extension $v$ of $u$ has all the required properties, namely satisfy Lemma 5.1 items 1,3 and 4 as well as (7.1) and (7.2) (with $w_{j}$ replaced by $v$ ). Such an extension is defined as follows. We let $\rho$ be a standard mollifier ${ }^{36}$ and set

$$
v(x, t)=\left\{\begin{array}{ll}
u * \rho_{t}(x), & \text { if } t>0 \\
u(x), & \text { if } t=0
\end{array} .\right.
$$

Clearly, items 1 and 4 in Lemma 5.1 hold true for this $v$. On the other hand, (7.2) is straightforward (when $|u| \leq 1$ ). In addition, the mapping $u \mapsto v$ is linear. By standard trace theory, this implies that items 2 and 4 yield item 3. In conclusion, we only need to prove (7.1). Before proceeding to the proof, we note that, by a standard limiting procedure, we may also assume $u$ smooth and compactly supported in $B^{m}$.

The proof of Lemma 5.1 relies on Hardy's inequality [39, Lemma 3.14] that we recall here.

${ }^{35}$ Indeed, an inspection of the proof below shows that when the map $u$ in Lemma 5.1 is replaced by $u-f_{\mathbb{S}^{m}} u$, then the corresponding map $v$ is replaced by $v-f_{\mathbb{S}^{m}} u$.

${ }^{36}$ That is, $\rho \in C_{c}^{\infty}\left(B^{m}\right), \rho \geq 0$ and $\int \rho=1$. 
7.1 Lemma. Let $p \geq 1$ and $r>1$. If $g \in W_{\text {loc }}^{1,1}([0, \infty))$, then

$$
\int_{0}^{\infty} \frac{|g(s)-g(0)|^{p}}{s^{r}} d s \leq\left(\frac{p}{r-1}\right)^{p} \int_{0}^{\infty} \frac{\left|g^{\prime}(s)\right|^{p}}{s^{r-p}} d s .
$$

7.2 Corollary. Let $p>1$ and $r>m$. If $w \in C^{1}\left(\mathbb{R}^{m}\right)$, then

$$
\int_{|y-x|<\rho} \frac{|w(y)-w(x)|^{p}}{|y-x|^{r}} d y \leq C \int_{|y-x|<\rho} \frac{|\nabla w(y)|^{p}}{|y-x|^{r-p}} d y, \quad \forall x \in \mathbb{R}^{m},
$$

where $C=C(p, r, m)$.

Proof. By replacing $g^{\prime}$ with $g^{\prime} \mathbb{1}_{[0, \rho]}$, we see that $(7.5)$ still holds when $[0, \infty)$ is replaced by $[0, \rho]$.

We have

$$
\begin{aligned}
\int_{|y-x|<\rho} \frac{|w(y)-w(x)|^{p}}{|y-x|^{r}} d y & =\int_{\mathbb{S}^{m-1}}\left(\int_{0}^{\rho} t^{m-r-1}|w(x+t \theta)-w(x)|^{p} d t\right) d \mathscr{H}^{m-1} \\
& \leq C \int_{|y-x|<\rho} \frac{|\nabla w(y)|^{p}}{|y-x|^{r-p}} d y
\end{aligned}
$$

the last inequality following from (7.5) applied in $[0, \rho]$ to $s \mapsto w(x+s \theta)$.

Proof of (7.1) when $u \in C_{c}^{\infty}\left(B^{m} ; \mathbb{R}^{k}\right)$. Let $v$ be given by (7.4).

Step 1. Pointwise estimates for the derivatives. The identity

$$
\partial_{j} v(x, t)=\frac{1}{t} u *\left(\eta_{j}\right)_{t}(x), \quad \forall x \in \mathbb{R}^{m}, t>0, j \in \llbracket 1, m+1 \rrbracket,
$$

where

$$
\eta_{j}=\partial_{j} \rho, \forall j \in \llbracket 1, m \rrbracket, \eta_{m+1}(x)=-\operatorname{div}(x \rho)(x),
$$

combined with the fact that $\int \eta_{j}=0, \forall j \in \llbracket 1, m+1 \rrbracket$, leads to

$$
|\nabla v(x, t)| \leq \frac{C}{t^{m+1}} \int_{|y-x|<t}|u(y)-u(x)| d y
$$

and thus

$$
|\nabla v(x, t)|^{p} \leq \frac{C}{t^{m+p}} \int_{|y-x|<t}|u(y)-u(x)|^{p} d y .
$$

Similarly, for the second order derivatives we have

$$
\left|D^{2} v(x, t)\right| \leq \frac{C}{t^{m+2}} \int_{|x-y|<t}|u(y)-u(x)| d y
$$

and

$$
\left|D^{2} v(x, t)\right|^{p} \leq \frac{C}{t^{m+2 p}} \int_{|y-x|<t}|u(y)-u(x)|^{p} d y .
$$

Step 2. $L^{p}$ estimate. By (7.4) and Hölder's inequality, we have

$$
|v(x, t)-u(x)|^{p} \leq \int_{|y-x|<t}|u(y)-u(x)|^{p} \rho_{t}(x-y) d y \leq \frac{C}{t^{m}} \int_{|y-x|<t}|u(y)-u(x)|^{p} d y .
$$


This implies

$$
\begin{aligned}
\int_{0}^{\infty} \int_{\mathbb{R}^{m}}|v(x, t)-u(x)|^{p} d x d t & \leq C \int_{0}^{\infty} \frac{1}{t^{m}} \iint_{|y-x|<t}|u(y)-u(x)|^{p} d y d x d t \\
& \leq C \int_{\mathbb{R}^{m}} \int_{\mathbb{R}^{m}}|u(y)-u(x)|^{p} \int_{|y-x|}^{\infty} \frac{d t}{t^{m}} d x d y \\
& =C \int_{\mathbb{R}^{m}} \int_{\mathbb{R}^{m}} \frac{|u(y)-u(x)|^{p}}{|y-x|^{m-1}} d x d y \leq C|u|_{W^{(k-1) / p, p}}^{p}
\end{aligned}
$$

the last estimate relies on Poincaré's inequality $\|u\|_{W^{(k-1) / p, p}} \leq C|u|_{W^{(k-1) / p, p}}$.

Hence, for every $T>0$ we have

$$
\|v\|_{L^{p}\left(\mathbb{R}^{m} \times(0, T)\right)} \leq C_{T}|u|_{W^{(k-1) / p, p}} .
$$

Step 3. $W^{1, p}$ estimate. As in the previous step, when $k \geq p$ (7.7) combined with Hölder's inequality implies

$$
\begin{aligned}
\|\nabla v\|_{L^{p}}^{p} & \leq C \int_{0}^{\infty} \int_{\mathbb{R}^{m}}\left(\int_{|y-x|<t}|u(y)-u(x)| d y\right)^{p} d x \frac{d t}{t^{(m+1) p}} \\
& \leq C \int_{0}^{\infty} \iint_{|y-x|<t}|u(y)-u(x)|^{p} d y d x \frac{d t}{t^{m+p}}=C \int_{\mathbb{R}^{m}} \int_{\mathbb{R}^{m}} \frac{|u(y)-u(x)|^{p}}{|y-x|^{m+p-1}} d y d x .
\end{aligned}
$$

Using again Poincaré's inequality, we find that

$$
\|\nabla v\|_{L^{p}}^{p} \leq C|u|_{W^{(k-1) / p, p}}^{p} .
$$

In particular, we have (by combining (7.13) with (7.14))

$$
\|v\|_{W^{1, p}\left(\mathbb{R}^{m} \times(0, T)\right)} \leq C_{T}|u|_{W^{(k-1) / p, p}},
$$

and the case where $k=p$ is completely settled.

For further use, let us note that the proof of (7.14) leads to

$$
\int_{0}^{\infty} \int_{\mathbb{R}^{m}} \frac{|\nabla v(x, t)|^{p}}{t^{k-p}} d x d t \leq C|u|_{W^{(k-1) / p, p}}^{p} .
$$

In order to estimate $|v|_{W^{k / p, p}}$ when $k \neq p$, we consider two different cases: $p>k$ and $p \in$ $(k-1, k)$.

Step 4. Estimate of $|v|_{W^{k / p, p}}$ when $p>k$. We start from the following semi-norms equivalence, valid for $1 \leq p<\infty$ and $0<\sigma<1:^{37}$

$$
\begin{aligned}
|v|_{W^{\sigma, p}\left(\mathbb{R}^{m} \times(0, \infty)\right)}^{p} & \int_{0}^{\infty} \int_{\mathbb{R}^{m}} \int_{\mathbb{R}^{m}} \frac{|v(y, t)-v(x, t)|^{p}}{|y-x|^{m+\sigma p}} d y d x d t \\
& +\int_{\mathbb{R}^{m}} \int_{0}^{\infty} \int_{0}^{\infty} \frac{|v(x, s+t)-v(x, t)|^{p}}{s^{1+\sigma p}} d s d t d x .
\end{aligned}
$$

This leads to

$$
|v|_{W^{k / p, p}}^{p} \sim I+J+K,
$$

where

$$
\begin{aligned}
& I:=\int_{0}^{\infty} \int_{\mathbb{R}^{m}} \int_{|y-x|<t} \frac{|v(y, t)-v(x, t)|^{p}}{|y-x|^{m+k}} d y d x d t, \\
& J:=\int_{0}^{\infty} \int_{\mathbb{R}^{m}} \int_{|y-x| \geq t} \frac{|v(y, t)-v(x, t)|^{p}}{|y-x|^{m+k}} d y d x d t,
\end{aligned}
$$

\footnotetext{
${ }^{37}$ As for (6.47), this is obtained by combining [1, Example 7.27] with [1, Lemma 7.44].
} 


$$
K:=\int_{\mathbb{R}^{m}} \int_{0}^{\infty} \int_{0}^{\infty} \frac{|v(x, s+t)-v(x, t)|^{p}}{s^{1+k}} d s d t d x .
$$

Using successively Corollary 7.2 and (7.8), we find that

$$
\begin{aligned}
I & \leq C \int_{0}^{\infty} \int_{\mathbb{R}^{m}} \int_{|y-x|<t} \frac{|\nabla v(y, t)|^{p}}{|y-x|^{m+k-p}} d y d x d t \\
& \leq C \int_{0}^{\infty} \frac{1}{t^{m+p}} \int_{\mathbb{R}^{m}} \int_{|y-x|<t} \frac{1}{|y-x|^{m+k-p}} \int_{|z-y|<t}|u(z)-u(y)|^{p} d z d y d x d t \\
& =C \int_{\mathbb{R}^{m}} \int_{\mathbb{R}^{m}} \int_{|z-y|}^{\infty} \frac{1}{t^{m+p}} \int_{|x-y|<t} \frac{1}{|x-y|^{m+k-p}} d x d t|u(z)-u(y)|^{p} d z d y \\
& =C \int_{\mathbb{R}^{m}} \int_{\mathbb{R}^{m}} \frac{|u(z)-u(y)|^{p}}{|z-y|^{m+k-1}} d z d y=|u|_{W^{(k-1) / p, p}}^{p} .
\end{aligned}
$$

The last two lines use Fubini's theorem. Similar calculations appear in the estimates of $J$ and $K$ and will not be detailed.

We next estimate $J$. Starting from

$$
|v(y, t)-v(x, t)| \leq|v(y, t)-u(y)|+|u(y)-u(x)|+|v(x, t)-u(x)|
$$

and using (7.11), we find that

$$
J \leq C\left(J_{1}+J_{2}\right),
$$

where

$$
J_{1}=\int_{0}^{\infty} \int_{\mathbb{R}^{m}} \int_{|y-x| \geq t} \frac{|u(y)-u(x)|^{p}}{|y-x|^{m+k}} d y d x d t=|u|_{W^{(k-1) / p, p}}^{p}
$$

and

$$
J_{2}=\int_{0}^{\infty} \frac{1}{t^{m}} \int_{\mathbb{R}^{m}} \int_{|y-x| \geq t} \frac{1}{|y-x|^{m+k}} \int_{|z-x|<t}|u(z)-u(x)|^{p} d z d y d x d t=C|u|_{W^{(k-1) / p, p}}^{p} .
$$

Finally, we estimate $K$ by using successively (7.5) applied with $g(s)=v(x, s+t)$ and (7.8). We find that

$$
\begin{aligned}
K & \leq C \int_{\mathbb{R}^{m}} \int_{0}^{\infty} \int_{0}^{\infty} \frac{|\nabla v(x, s+t)|^{p}}{s^{1+k-p}} d s d t d x \\
& \leq C \int_{\mathbb{R}^{m}} \int_{0}^{\infty} \frac{1}{s^{1+k-p}} \int_{0}^{\infty} \frac{1}{(s+t)^{m+p}} \int_{|y-x|<s+t}|u(y)-u(x)|^{p} d y d s d t d x=C|u|_{W^{(k-1) / p, p}}^{p} .
\end{aligned}
$$

We complete the case where $p>k$ by combining (7.18)-(7.23).

Step 5. Estimate of $|v|_{W^{k / p, p}}$ when $p \in(k-1, k)$. Starting from $|v|_{W^{k / p, p}}=|\nabla v|_{W^{k / p-1, p}}$ and (7.17), we find that

$$
|v|_{W^{k / p, p}}^{p} \sim \widetilde{I}+\widetilde{J}+\widetilde{K}
$$

where

$$
\begin{aligned}
& \widetilde{I}:=\int_{0}^{\infty} \int_{\mathbb{R}^{m}} \int_{|y-x|<t} \frac{|\nabla v(y, t)-\nabla v(x, t)|^{p}}{|y-x|^{m+k-p}} d y d x d t, \\
& \widetilde{J}:=\int_{0}^{\infty} \int_{\mathbb{R}^{m}} \int_{|y-x| \geq t} \frac{|\nabla v(y, t)-\nabla v(x, t)|^{p}}{|y-x|^{m+k-p}} d y d x d t, \\
& \widetilde{K}:=\int_{\mathbb{R}^{m}} \int_{0}^{\infty} \int_{0}^{\infty} \frac{|\nabla v(x, s+t)-\nabla v(x, t)|^{p}}{s^{1+k-p}} d s d t d x .
\end{aligned}
$$

In order to estimate the quantities $\widetilde{I}$ and $\widetilde{K}$, we proceed as for $I$ and $K$, but use (7.10) instead of (7.8). Finally, using (7.16) we obtain

$$
\widetilde{J} \leq C \int_{0}^{\infty} \int_{\mathbb{R}^{m}} \int_{|y-x| \geq t} \frac{|\nabla v(x, t)|^{p}}{|y-x|^{m+k-p}} d y d x d t=C \int_{0}^{\infty} \int_{\mathbb{R}^{m}} \frac{|\nabla v(x, t)|^{p}}{t^{k-p}} d x d t \leq C|u|_{W^{(k-1) / p, p}}^{p} .
$$

This completes the proof of Lemma 5.1. 


\section{References}

[1] R.A. Adams. Sobolev spaces. Academic Press [A subsidiary of Harcourt Brace Jovanovich, Publishers], New York-London, 1975. Pure and Applied Mathematics, Vol. 65.

[2] G. Alberti, S. Baldo, and G. Orlandi. Functions with prescribed singularities. J. Eur. Math. Soc. (JEMS), 5(3):275-311, 2003.

[3] F. Almgren, F. Browder, and E.H. Lieb. Co-area, liquid crystals, and minimal surfaces. In Partial differential equations (Tianjin, 1986), volume 1306 of Lecture Notes in Math., pages 1-22. Springer, Berlin, 1988.

[4] J.M. Ball. Convexity conditions and existence theorems in nonlinear elasticity. Arch. Rational Mech. Anal., 63(4):337-403, 1976/77.

[5] F. Bethuel. A characterization of maps in $H^{1}\left(B^{3}, S^{2}\right)$ which can be approximated by smooth maps. Ann. Inst. H. Poincaré Anal. Non Linéaire, 7(4):269-286, 1990.

[6] F. Bethuel. The approximation problem for Sobolev maps between two manifolds. Acta Math., 167(3-4):153-206, 1991.

[7] F. Bethuel, J.-M. Coron, F. Demengel, and F. Hélein. A cohomological criterion for density of smooth maps in Sobolev spaces between two manifolds. In Nematics (Orsay, 1990), volume 332 of NATO Adv. Sci. Inst. Ser. C Math. Phys. Sci., pages 15-23. Kluwer Acad. Publ., Dordrecht, 1991.

[8] F. Bethuel and X.M. Zheng. Density of smooth functions between two manifolds in Sobolev spaces. J. Funct. Anal., 80(1):60-75, 1988.

[9] J. Bourgain, H. Brezis, and P. Mironescu. $H^{1 / 2}$ maps with values into the circle: minimal connections, lifting, and the Ginzburg-Landau equation. Publ. Math. Inst. Hautes Études Sci., 99:1-115, 2004.

[10] J. Bourgain, H. Brezis, and P. Mironescu. Lifting, degree, and distributional Jacobian revisited. Comm. Pure Appl. Math., 58(4):529-551, 2005.

[11] P. Bousquet. Topological singularities in $W^{s, p}\left(S^{N}, S^{1}\right)$. J. Anal. Math., 102:311-346, 2007.

[12] P. Bousquet, A.C. Ponce, and J. Van Schaftingen. Density in $W^{m, p}, 2011$.

[13] H. Brezis, J.-M. Coron, and E.H. Lieb. Harmonic maps with defects. Comm. Math. Phys., 107(4):649-705, 1986.

[14] H. Brezis and P. Mironescu. Gagliardo-Nirenberg, composition and products in fractional Sobolev spaces. J. Evol. Equ., 1(4):387-404, 2001. Dedicated to the memory of Tosio Kato.

[15] H. Brezis and P. Mironescu. Density in $W^{s, p}, 2012$.

[16] H. Brezis, P. Mironescu, and A. Ponce. $W^{1,1}$-maps with values into $S^{1}$. In Geometric analysis of PDE and several complex variables, volume 368 of Contemp. Math., pages 69-100. Amer. Math. Soc., Providence, RI, 2005.

[17] H. Brezis and L. Nirenberg. Degree theory and BMO. I. Compact manifolds without boundaries. Selecta Math. (N.S.), 1(2):197-263, 1995.

[18] A. Cohen, W. Dahmen, I. Daubechies, and R. DeVore. Harmonic analysis of the space BV. Rev. Mat. Iberoamericana, 19(1):235-263, 2003.

[19] H. Federer. Geometric measure theory. Die Grundlehren der mathematischen Wissenschaften, Band 153. Springer-Verlag New York Inc., New York, 1969.

[20] H. Federer and W.H. Fleming. Normal and integral currents. Ann. of Math. (2), 72:458$520,1960$.

[21] M. Giaquinta, G. Modica, and J. Souček. Cartesian currents in the calculus of variations. I, volume 37 of Ergebnisse der Mathematik und ihrer Grenzgebiete. 3. Folge. A Series of Modern Surveys in Mathematics [Results in Mathematics and Related Areas. 3rd Series. A Series of Modern Surveys in Mathematics]. Springer-Verlag, Berlin, 1998. Cartesian currents. 
[22] F.B. Hang and F.H. Lin. A remark on the Jacobians. Commun. Contemp. Math., 2(1):35$46,2000$.

[23] R. Hardt, D. Kinderlehrer, and F.H. Lin. Stable defects of minimizers of constrained variational principles. Ann. Inst. H. Poincaré Anal. Non Linéaire, 5(4):297-322, 1988.

[24] R. Hardt and F.H. Lin. Mappings minimizing the $L^{p}$ norm of the gradient. Comm. Pure Appl. Math., 40(5):555-588, 1987.

[25] R.L. Jerrard and H.M. Soner. Functions of bounded higher variation. Indiana Univ. Math. J., 51(3):645-677, 2002.

[26] R.L. Jerrard and H.M. Soner. The Jacobian and the Ginzburg-Landau energy. Calc. Var. Partial Differential Equations, 14(2):151-191, 2002.

[27] J. Lafontaine. Introduction aux variétés différentiables. EDP Sciences, Grenoble, 2010.

[28] M. Escobedo. Some remarks on the density of regular mappings in Sobolev classes of $S^{M}$-valued functions. Rev. Mat. Univ. Complut. Madrid, 1(1-3):127-144, 1988.

[29] P. Mironescu. $\mathbb{S}^{1}$-valued Sobolev mappings. J. Math. Sci. (N. Y.), 170(3):340-355, 2010.

[30] P. Mironescu. Sobolev spaces of circle-valued maps, 2012.

[31] I. Molnar. Prescribed singularities with weights, 2012.

[32] F. Morgan. Geometric measure theory. Elsevier/Academic Press, Amsterdam, fourth edition, 2009. A beginner's guide.

[33] L. Nirenberg. On elliptic partial differential equations. Ann. Scuola Norm. Sup. Pisa (3), 13:115-162, 1959.

[34] M. R. Pakzad and T. Rivière. Weak density of smooth maps for the Dirichlet energy between manifolds. Geom. Funct. Anal., 13(1):223-257, 2003.

[35] A.C. Ponce and J. Van Schaftingen. Closure of smooth maps in $W^{1, p}\left(B^{3} ; S^{2}\right)$. Differential Integral Equations, 22(9-10):881-900, 2009.

[36] T. Rivière. Dense subsets of $H^{1 / 2}\left(S^{2}, S^{1}\right)$. Ann. Global Anal. Geom., 18(5):517-528, 2000.

[37] R. Schoen and K. Uhlenbeck. A regularity theory for harmonic maps. J. Differential Geom., 17(2):307-335, 1982.

[38] R. Schoen and K. Uhlenbeck. Boundary regularity and the Dirichlet problem for harmonic maps. J. Differential Geom., 18(2):253-268, 1983.

[39] E.M. Stein and G. Weiss. Introduction to Fourier analysis on Euclidean spaces. Princeton University Press, Princeton, N.J., 1971. Princeton Mathematical Series, No. 32. 